BABELAO 6 (2017), p. 75-124

(C) ABELAO (Belgium)

\title{
Les versions syriaques des Dis- cours de Grégoire de Nazianze : un processus continu de révision
}

Par

\section{Jean-Claude Haelewyck}

Université de Louvain, Louvain-la-Neuve

$\mathrm{L}$

orsqu'il meurt en 390, Grégoire de Nazianze laisse à la postérité une œuvre unifiée et bien circonscrite ${ }^{1}$. Sans doute a-t-il veillé avant de mourir à constituer lui-même le corpus des œuvres qu'il désirait transmettre. Cet ensemble contient 45 Discours, plus de 240 lettres $^{2}$, ainsi que de nombreux poèmes théologiques et historiques dont un poème auto-

\footnotetext{
${ }^{1}$ Une version abrégée de cet article a été présentée à Rome le 20 février 2017 comme communication au Colloque Tradurre tradire tramandare : $i$ Padre Greci nell'Occidente Latino e nell'Oriente Siriaco - 20-21/02/2017, Università degli Studi, Roma Tre.

${ }^{2}$ Les Lettres ne sont pas éditées en syriaque. Dans cet ensemble de lettres, il faut mettre à part les lettres théologiques (ep. 101, 102 et 202). Il semble qu'on ait conservé plusieurs versions syriaques pour ce qui est des ep. $101 \mathrm{et}$ 102, mais pas en intégralité pour l'ep. 101 ; en revanche pour l'ep. 202 il n'y aurait qu'une seule version. La version syriaque des lettres théologiques fait l'objet d'une thèse entreprise à l'Institut Orientaliste par Mme Sophie Meunier (qui m'a bien aimablement communiqué les informations reprises ici).
} 
biographique (Carm. 11). L'authenticité de sa tragédie (le Christus patiens) est parfois mise en doute. La majorité des écrits qui nous sont parvenus date de la fin de sa vie. Je n'envisagerai ici que la question de la traduction des Discours (Orationes).

\section{Présentation des versions anciennes des Discours de Grégoire de Nazianze}

À peine 10 ans se sont écoulés après la mort de Grégoire que Rufin d'Aquilée traduit en latin neuf de ses Discours : 2, 6, 16, $17,26,27,38,39$ et $41^{3}$. La traduction inaugure la notoriété dont jouira Grégoire dans la partie latine de l'Empire et conserve les traces d'un état ancien de la tradition (cf. infra). On a depuis longtemps souligné la liberté de Rufin : il introduit des modifications grammaticales (personnes et voix des verbes), il réélabore les périodes, il ajoute, supprime ou déplace des éléments, laisse transparaître ses préoccupations propres ${ }^{4}$.

Très vite dans le courant du $5^{\mathrm{e}}$ siècle, bon nombre des Discours vont être traduits dans les langues orientales. La tradition copte n'a conservé que quelques textes, dans deux dialectes : en sahidique le copte transmet des fragments des Discours 21, 43 et 45, et en bohaïrique le texte complet du Discours $14^{5}$.

C'est en arménien que le plus ancien corpus complet des Discours nous a été conservé, et dans 150 manuscrits. La traduction est anonyme et il est impossible de la dater ou de la localiser avec précision. Toutefois une analyse des techniques de traduction montre que la langue utilisée est antérieure à l'école hellénophile arménienne (datée du $6^{\mathrm{e}} \mathrm{s}$.). La traduction a dû être réalisée entre 500 et 550 . La traduction arménienne est très fidèle, voire littérale. Elle permet donc de se faire une idée précise du texte grec modèle. En arménien les Discours suivants ont été édités : $2,4,5,6,7,8,9,12,21^{6}$.

${ }^{3}$ A. ENGELBRECht, Tyrannii Rufini Orationum Gregorii Nazianzeni novem interpretatio. Les références complètes sont données dans la bibliographie finale.

${ }^{4}$ Les Actes du colloque de Rome (voir n. 1) contiendront deux contributions importantes sur ce point : l'une de Sabrina Antonella Robbe sur Rufin traducteur de l'Histoire ecclésiastique d'Eusèbe, l'autre de Carla Lo Cicero sur Rufin traducteur de Basile.

${ }^{5}$ Le tout a été édité. G. LAFONTAINE, « La version copte sahidique du Discours 'Sur la Pâque' de Grégoire de Nazianze »; "La version copte bohaïrique du Discours 'Sur l'amour des pauvres' de Grégoire de Nazianze »; «La version copte des Discours de Grégoire de Nazianze ». Voir G. LAFONTAINE - H. METREVELI, «Les versions copte, arménienne et géorgienne de Saint Grégoire le Théologien. Etat des recherches ».

${ }^{6}$ Dans le Corpus Nazianzenum ont paru les Discours 2, 12 et 9 (édités par B. Coulie en 1994), 4 et 5 (par A. Sirinian en 1999), 21, 7 et 8 (par B. Coulie et A. Sirinian en 1999), 6 (par C. Sanspeur en 2007). 
La version géorgienne est d'une grande complexité. Non seulement elle regroupe un peu plus de 200 témoins manuscrits, mais certains textes de Grégoire ont fait l'objet d'une quintuple traduction. On distingue ainsi une version anonyme de deux Discours (38 et 39) réalisée entre le $7^{\mathrm{e}}$ et le $9^{\mathrm{e}}$ s. et intégrée dans des homéliaires (Mravaltavi). Dans la seconde moitié du $10^{\mathrm{e}} \mathrm{s}$, Grégoire d'Ochki a traduit les Discours 7 et 27. Il est le seul à avoir traduit à partir de l'arménien; les autres se sont basés sur le grec. À la même époque, David Tbeli a traduit 11 textes (Discours 2, 4, 8, 9, 10, 12, 24, 26, 28, 34, Ep. 101). Au Mont Athos, entre 975 et 1028, Euthyme l'Hagiorite traduit 22 Discours. Enfin 17 Discours sont traduits par Éphrem Mtsire ou Éphrem le Petit au $11^{\mathrm{e}} \mathrm{s}$. au monastère de la Montagne Noire près d'Antioche. Jusqu'à présent ${ }^{7}$ onze Discours ont été édités $(1,11$, $15,19,21,24,38,39,40,41,42,43,44,45)$, parfois avec une publication en parallèle des différentes versions.

Dans la tradition arabe, les Discours de Grégoire ont été conservés sous la double forme d'une collection de 30 pièces et de Discours isolés dans des homéliaires et autres recueils. En partant d'une étude des Discours de la collection grecque de XVI lus $^{8}$, on peut proposer l'histoire suivante. Dans un premier temps, il est probable que quelques discours aient été traduits isolément (version assez fruste du Discours 38 dans un témoin $\mathrm{du} 10^{\mathrm{e}} \mathrm{s}$.). Un premier traducteur a traduit une collection de XVI complétée reposant sur un texte grec de type $n-x^{9}$; elle concerne les Discours liturgiques (1, 38, 39, 40, 42, 44, 45, etc.), les laudatifs $(11,21,24)$ et probablement d'autres (dont le Discours 3). Elle serait l'œuvre d'un certain Antonios, moine à Saint Saba puis supérieur du monastère de Saint-Siméon le Thaumaturge, actif dans la seconde moitié du $10^{\mathrm{e}} \mathrm{s}$. La seconde est l'œuvre d'Ibrahim ibn Yuhanna al-Antaki (fin du $10^{\mathrm{e}} \mathrm{s}$.). Il entreprend à son tour la version arabe d'une collection de XVI : il reprend et révise la version précédente sur un texte grec de type $\mathrm{m}$. Sa révision consiste à supprimer les calques, préciser le lexique, éclaircir certains passages par le recours à des additions et doublets de traductions. Une petite collection circule sous son nom, et c'est

\footnotetext{
${ }^{7}$ Le Corpus Nazianzenum contient l'édition des Discours 1, 45, 44, 41 (par H. Metreveli et alii en 1998), 15, 24, 19 (par H. Metreveli et alii en 2000), 38 (par H. Metreveli et alii en 2001), 43 (par B. Coulie, H. Metreveli et alii en 2004), 39, 40 (par B. Coulie, H. Metreveli et alii en 2007), 11, 21 et 42 (par B. Coulie en 2013).

${ }^{8}$ Sur les collections grecques des Discours de Grégoire, voir V. SOMERS, Histoire des collections complètes, 1997. Je me suis très largement inspiré des notes que L. Tuerlinckx m'a aimablement transmises sur les versions arabes.

${ }^{9}$ Sur les familles de manuscrits grecs, voir les introductions aux éditions des Discours de Grégoire dans les Sources chrétiennes et V. SOMERS, Histoire des collections complètes, 1997. Voir aussi plus loin.
} 
de ce fonds que proviennent les discours repris dans les homéliaires et ménologes, ce qui explique que les discours dits isolés, témoins de cet état de texte, présentent souvent des lectures distinctes de celle des collections (voir ci-après), mais plus proches de l'original grec. En effet, les collections reflètent un stade ultérieur de l'histoire du texte : un compilateur réunit les discours traduits ou révisés par Ibrahim, ceux présumés d'Antonios qui n'ont pas connu de révision, pour composer la collection partielle de 30 pièces telles qu'elle nous est parvenue dans une quinzaine de manuscrits du $13^{\mathrm{e}}$ au $19^{\mathrm{e}}$ s. (25 homélies, 2 lettres, 2 poèmes et la $\mathrm{Vita}$ ). L'ensemble a été révisé de façon interne à l'arabe (pour uniformiser le texte sur les plans syntaxiques et morphologiques), mais sans modifier le lexique ou les expressions d'origine, de sorte que les caractéristiques propres de chaque traducteur ont été conservées dans l'un ou l'autre discours. La tradition manuscrite comprend près de 160 témoins ${ }^{10}$. Le Sinaï a joué un rôle important dans la transmission des versions arabes. Jusqu'à présent les Discours suivants ont été édités en arabe : $1,11,21,40,41,44,45^{11}$.

La tradition slave est complexe, et seule une étude de l'ensemble de la tradition manuscrite permettra de répondre aux nombreuses questions qui restent en suspens. Trois traductions sont attestées. Une première version de XIII Discours a été réalisée au $10^{\mathrm{e}}-11^{\mathrm{e}} \mathrm{s} .{ }^{12}$ Une deuxième version faite à la même

${ }^{10}$ Le regroupement de ces témoins manuscrits varie d'un Discours à l'autre. J. Grand'Henry (qui m'a bien aimablement aidé pour rédiger ce qui suit) a isolé trois familles : une famille syro-sinaïtique ( $x$, à subdiviser en proto-version syrienne, en famille syro-libanaise et famille sinaitique), une famille égyptienne $(y)$ et une famille intermédiaire (entre $x$ et $y$, mais plus proche de $y$ que de $x$ ). La situation est trop complexe pour qu'elle puisse être ici exposée. Tous les détails peuvent être lus dans les introductions des Discours de Grégoire en tradition arabe déjà parus (en particulier les volumes de J. Grand'Henry).

11 Le Corpus Nazianzenum contient l'édition des Discours 21 (par J. Grand'Henry en 1996), 1, 45, 44 (par L. Tuerlinckx en 2001), 40 (par J. Grand'Henry en 2005), 11, 41 (par Grand'Henry en 2013). Je remercie L. Tuerlinckx et J. Grand'Henry qui m'ont aidé à rédiger le paragraphe sur les versions arabes.

${ }^{12}$ La collection de «XIII Discours de Grégoire le Théologien » figure dans un codex du $11^{\mathrm{e}} \mathrm{s}$. conservé à la Bibliothèque nationale de Russie de Saint-Pétersbourg ( $\mathrm{n}^{\circ}$ Q. p. I. 16). La sélection et l'ordre des homélies (39, $43,40,38,2,6,5,27,28,16,45,41,1)$ diffèrent de ceux des modèles grecs connus. Ces homélies ont été traduites par plusieurs traducteurs qui ont travaillé à des moments différents et dans des centres différents aux $10^{\mathrm{e}}-11^{\mathrm{e}} \mathrm{s}$. dans la région sud-slave (Preslav en Bulgarie et Ohrid en Macédoine). Une analyse du rendu de la terminologie grecque et de l'évolution de la langue cible permet de conclure à une formation graduelle de cette collection qui est non seulement la plus ancienne traduction d'œuvres de Grégoire en langue slave, mais encore une des plus anciennes collections slaves de textes non liturgiques. Les XIII homélies ont été édités par A. BUDILOVICH, XIII slov Grigoriya Bogoslova, Saint-Pétersbourg, 1875. Voir Fr.J. THOMSON, «The 
époque est connue par des mss du $14^{\mathrm{e}} \mathrm{s}$. Elle utilise et corrige 8 Discours de la première version et en traduit sur nouveaux frais 8 autres $^{13}$. Une troisième rédaction/révision a été opérée au cours des $15^{\mathrm{e}}-16^{\mathrm{e}}$ siècles. Il n'existe pas d'édition critique scientifique des Discours des deux dernières rédactions en slavon ${ }^{14}$.

En éthiopien, on ne connaît que des citations éparses dans trois florilèges monophysites des $15^{\mathrm{e}}$ et $16^{\mathrm{e}} \mathrm{s}$. La traduction éthiopienne a été réalisée à partir d'une version arabe, ellemême traduite du copte ${ }^{15}$. Sauf erreur de ma part, rien n'a été publié jusqu'à présent pour l'éthiopien.

\section{Les versions syriaques des Discours de Grégoire de Na- zianze}

Venons-en maintenant au syriaque en commençant par un bref état des questions ${ }^{16}$. Les premières informations concernant

works of St. Gregozy of Nazianzus in Slavonic », 1983 et M. GRASSELLINI, « La tradizione slava delle omelie di S. Gregorio di Nazianzino », 1992.

${ }^{13}$ Ces manuscrits confectionnés en Bulgarie ou au Mont-Athos, datant du $14^{\mathrm{e}}$ s., contiennent «XVI Discours de St Grégoire le Théologien avec un commentaire du métropolite Nicetas d'Héraclée » $(38,39,40,45,1,44,41$, $14,16,42,15,43,21,24,19,11)$. Il s'agit d'une nouvelle rédaction, à partir du grec, de 8 Discours contenus dans la collection précitée $(10,16,38,40$, 41 , 43 et 45 ), ainsi que de 8 autres qui n'y figurent pas et dont les archaïsmmes font également remonter la traduction aux $10^{\mathrm{e}}-11^{\mathrm{e}} \mathrm{s}$. Cette collection a été éditée à Moscou en 1680 par Evfimij Čudovskij; elle est accompagnée de commentaires du métropolite Nicétas d'Héraclée [григория богослова 16 слов с толкованиями митрополита Никиты Ираклийскаго - Grigorija Bogoslova 16 Slov s tolkovanijami mitropolita Nikity Iraklijskago - Grégoire le Théologien, 16 Discours avec des commentaires du métropolite d'Héraclée].

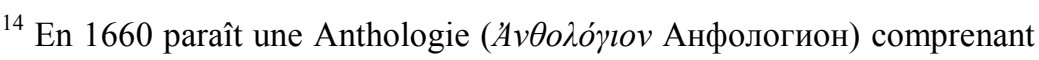
les Tetrastichae Sententiae traduites par Arsène le Grec. le Discours sur la Pentecôte a été publié à Moscou dans un recueil de 71 homélies de Pères. En 1798 l'évêque Irenej Klement'evskij publie à Moscou (Typographie synodale) en deux volumes sa traduction de Discours, Homélies et Lettres, avec des commentaires de l'Ecclésiaste du métropolite Nicetas d'Héraclée [Святаго Григория Богослова, Архиепископа Константина Града Поучительных Слов - Svjatago Grigorija Bogoslova, arhiepiskopa Konstantina Grada Poučitel'nyh Slov - S. Grégoire le Théologien, arche-vêque de Constantinople, Discours instructifs). Entre 1822 et 1848, une sélection de Discours et d'autres ouvres de Grégoire a été éditée dans la collection Lectures chrétiennes publiée par l'Académie de théologie de Moscou. Entre 1843 et 1848 ont été éditées à Moscou en 6 volumes les Euvres de notre saint père Grégoire le Théologien: Творения иже во святых отиа нашего Григория Богослова [Tvorenija iže vo svjatyh otca našego Grigorija Bogoslova - Euvres de notre saint père Grégoire le Théologien]. Moscou (plusieurs rééditions). Je dois tous ces renseignements récents concernant le slave à M. Pirard que je remercie vivement.

${ }^{15}$ E. van DONZEL, « Les versions éthiopiennes ».

${ }^{16}$ Voir J.-Cl. HAELEWYCK, Oratio XL, p. vi-xiii. 
la traduction syriaque des œuvres de Grégoire de Nazianze remontent à Barhebraeus (mort en 1286) et à Abdisho (mort en 1318). Le premier signale que les œuvres de Grégoire étaient réparties en syriaque sur deux tomes contenant 47 Discours et 31 Lettres, et qu'il existait deux versions : une première, anonyme, et une seconde réalisée par Paul d'Édesse en 623/624 ${ }^{17}$. Le témoignage d'Abdisho ne concorde pas entièrement avec celui de Barhebraeus. Il affirme en effet que « Grégoire le Théologien, évêque de Nazianze, a cinq tomes et (des pièces) en mètres iambiques, (des) question(s) à Césaire, ainsi que la Tragédie, et encore le livre qu'il composa contre les Théopaschites $»^{18}$. A. Baumstark concilie les deux témoignages en supposant que Paul d'Édesse avait réuni en deux tomes les cinq de l'ancienne version ${ }^{19}$. Nous verrons qu'une autre interprétation est possible.

En 1725, dans sa notice sur Abdisho, Assemani reprend les affirmations de Barhebraeus et ajoute qu'il a pu en 1715 consulter au monastère de Deir-es-Suryan trois manuscrits de la version dite jacobite par Barhebraeus. Dans le colophon de l'un d'eux, dit-il, il a lu que les 30 Discours formant la première partie des œuvres de Grégoire avaient été traduits par Paul, évêque de Chypre en $624^{20}$. Ce qu'Assemani appelle « colophon » est en réalité une partie du titre et du pinax du $\mathrm{ms}^{21}$. De plus, Paul n'a pas été évêque de Chypre : Chypre est lieu où il s'est réfugié après l'invasion perse et où il a entrepris sa traduction/révision des Discours de Grégoire. Il est encore question de Paul d'Édesse ailleurs dans la tradition syriaque, en lien avec les Scholies mythologiques. Dans le courant du $6^{\mathrm{e}}$ s., un auteur grec anonyme a ajouté à quatre Discours une série de scholies destinées à expliciter les allusions de Grégoire à la mythologie païenne. Ces Scholies mythologiques sont passées dans la tradition syriaque, mais elles n'y sont conservées au complet que

${ }^{17}$ J.S. ASSEMANI, Bibliotheca Orientalis, II, p. 302 et 307 ; la notice est reprise en III/1, p. 23, n. 1. Barhebraeus attribuait la seconde version à Jacques d'Édesse. Aujourd'hui on la rattache à Paul d'Édesse. Mais voir plus loin ce qui peut être dit du rôle de Jacques d'Édesse. La première version sera dénommé ci-après $\mathrm{S} 1$ et la seconde $\mathrm{S} 2$.

18 J.S. ASSEMANI, Bibliotheca Orientalis, III/1, p. 23-24. Les « Questions à Césaire » (Erotapokriseis) sont inauthentiques et datent de la $1^{\mathrm{e}}$ moitié du $6^{\mathrm{e}} \mathrm{s}$. La traduction française des sept vers syriaques est celle de A. DE HALLEUX, « La version syriaque », p. 89, n.121.

\footnotetext{
19 A. BAUMSTARK, Geschichte, p. 77 ; S. BROCK, Pseudo-Nonnos, p. 8, n. 2 .

${ }^{20}$ J.S. ASSEMANI, Bibliotheca Orientalis, III/1, p. 23, n. 1 ; il renvoie là au tome I, p. 171-172 où il édite le colophon.

${ }^{21}$ Sur les abréviations des manuscrits syriaques des Discours de Grégoire de Nazianze, voir l'annexe.
} 
dans la version récente S2. À la fin des Scholies mythologiques du Discours 39 (sur l'Épiphanie), on peut lire dans les mss A B $\mathrm{C}$ E F et G la notice suivante : "Sont achevées les nouvelles scholies que l'honoré de Dieu et pieux Abas Mar Pola a corrigées $»^{22}$. Le verbe syriaque « corriger » $\left(5^{i \downarrow}\right)$ n'a probablement pas ici son sens propre: les auteurs syriaques ont tendance à employer indifféremment les verbes éditer (مص), interpréter (ara), réviser ( $5^{i}$ i ) ou traduire ( se retrouve aussi à propos des mentions de Jacques d'Édesse dans les manuscrits syriaques de Grégoire, en particulier dans les deux colophons du ms. A. Dans le premier, le scribe Aksenoyo affirme que Jacques d'Édesse a effectué « une correction

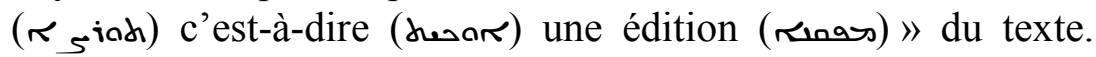
Pour Aksenoyo les deux termes sont donc synonymes. Dans le second colophon, il est dit que Jacques d'Édesse a édité (هas) les Discours et qu'Aksenoyo les a révisés ( 5 ił). Le témoignage du second colophon est à refuser : il est tardif et force le trait. En revanche, celui du premier peut trouver un appui dans le corps même du manuscrit : le ms. A contient en effet des notes marginales dont certaines sont attribuées à Jacques d'Édesse.

La tradition manuscrite a conservé des traces d'une révision supplémentaire de la version récente. Dans la version syriaque des Scholies mythologiques du Pseudo-Nonnos, le ms. C affirme (fol. 121r) que le texte de Paul d'Édesse a été révisé par le patriarche Athanase ${ }^{23}$, à savoir Athanase de Balad (mort en 686). Le témoignage du ms. C est confirmé par ceux de Georges des Arabes (mort en 724) et de Timothée $1^{\mathrm{er}}$ (mort en 823) qui mentionnent aussi une révision d'Athanase ${ }^{24}$. Une incertitude demeure toutefois : cette révision d'Athanase concerne-t-elle tous les Discours ou seulement les Scholies mythologiques, en particulier celles du Discours 39 ? La prudence exige qu'on la limite aux Scholies mythologiques ${ }^{25}$.

En 1913, W. Lüdtke esquisse 1'histoire de la transmission des œuvres de Grégoire de Nazianze en latin et dans les langues orientales. Retenons ce qu'il dit du syriaque ${ }^{26}$. En s'appuyant

${ }^{22}$ S. BRocK, Pseudo-Nonnos, p. 191 (texte syriaque) et 70 (traduction).

23 W. Wright, Catalogue, p. 425b; S. BROCK, « Überlieferungsgeschichte », p.461-462 ; ID., Pseudo-Nonnos, p. 191.

${ }^{24}$ S. BRock, Pseudo-Nonnos, p. 30-31 ; A. DE HALleuX, « La version syriaque $»$, p. 81 .

${ }^{25}$ Comme le fait S. Brock, Pseudo-Nonnos, p. 30-31. Des auteurs plus anciens avaient envisagé que la révision d'Athanase concernait tous les Discours : R. DuvAL, La littérature syriaque, Paris, 1907, p. 309 ; J.-B. CHABOT, Littérature syriaque, Paris, 1934, p. 83-84 ; I. ORTIZ DE URBINA, Patrologia syriaca, Rome, 1965, p. 183.

${ }^{26}$ W. LÜDTKE, « Zur Überlieferung », p. 264-270. 
sur les collations du début du Discours 45 dans les mss T et W, et de la totalité du Discours 1 dans les mss T B et C, il confirme l'existence de deux versions syriaques et apporte un élément nouveau: "Les deux versions ne sont pas mutuellement indépendantes : l'une est la révision de l'autre. S2 écarte les mots empruntés au grec, corrige des erreurs et rend de manière plus précise les subtilités de l'original ». Lüdtke analyse ensuite les citations de Grégoire chez les auteurs syriaques et montre que la première version est citée par les auteurs nestoriens Babaï le Grand (mort peu après 628) et Sahdona en 630, et que la version récente l'est chez le jacobite Athanase de Nisibe en 669. Lüdtke suggère que la version $\mathrm{S} 1$ était la version commune aux deux confessions syriaques et qu'elle fut remplacée, chez les Jacobites, par celle de Paul d'Édesse.

Les travaux de Brock $^{27}$ confirment et approfondissent les hypothèses de Lüdtke. D'après lui, la première traduction syriaque (S1) a été réalisée au $6^{\mathrm{e}} \mathrm{s}$. et subsiste dans les mss T et W. Les Discours auraient été répartis en cinq tomes (cf. Abdisho). En 623-624 Paul d'Édesse réalisa une révision complète de l'ancienne version. À cette occasion, les Discours furent répartis sur deux tomes. Cette révision de Paul subsiste dans sept mss du premier tome (A, B, C, D, E, F, G) mais dans un seul témoin du second $(\mathrm{P})$. Des traces d'une révision ultérieure, par Athanase de Balad, apparaissent dans quatre témoins du premier tome $(B, C$, E, F). Grégoire de Nazianze cessa d'être lu après le $9^{\mathrm{e}} \mathrm{s}$. comme l'indiquent les trois observations suivantes: aucun recueil des Discours n'a été composé après le $9^{\mathrm{e}} \mathrm{s}$., les commentaires des Discours commencent à être produits dès $850^{28}$, les citations sont rares chez les auteurs plus tardifs.

A. de Halleux met la touche finale à la recherche ${ }^{29}$. En ce qui concerne l'organisation des Discours dans la tradition syriaque, il revient sur la contradiction entre Barhebraeus (deux tomes) et Abdisho (cinq tomes). Plutôt que de considérer, avec S. Brock, que Paul d'Édesse a réparti en deux tomes l'ancienne répartition en cinq tomes, il suppose que la même collection, avec la même division et la même acolouthie (ou ordre des pièces), a existé dès le début. Il se fonde sur le témoignage du même Abdisho qui

27 S. BROCK, «Armenian and Syriac Version»; ID., «Überlieferungsgschichte »; ID., Pseudo-Nonnos, p. 15-33. Voir A. DE HALLEUX, « La version syriaque $»$, p. 85.

${ }^{28}$ Sur les commentaires, voir I. ISEBAERT-CAUUET, « Les Pères grecs », 2007, p. 84-86.

${ }^{29}$ Dans les deux articles suivants : A. DE HALlEUX, « L'homélie baptismale »; ID., « La version syriaque ». 
parle ailleurs des deux tomes de Grégoire ${ }^{30}$. Dès lors les cinq tomes pourraient représenter les cinq œuvres citées dans la notice (Orationes, Carmina, Erôtapocriseis, Christus patiens, lettres dogmatiques) ou, plus vraisemblablement, les cinq collections de Discours répartis de manière chronologique. Trois scholies sur la chronologie des Discours ${ }^{31}$ divisent en effet la vie de Grégoire en cinq périodes : "avant qu'il ne devienne prêtre », "lorsqu'il est prêtre », " dans sa ville après qu'il fut devenu évêque », " lorsqu'il fut monté à Constantinople » et « lorsqu'il fut revenu de Constantinople dans sa ville ». A. de Halleux s'interroge ensuite sur le rôle à attribuer à Jacques d'Édesse $\left(7^{\mathrm{e}}\right.$ s.). On ne peut se contenter, dit-il, de discréditer le témoignage de Barhebraeus ${ }^{32}$. Deux solutions sont possibles. Ou bien Jacques d'Édesse a, non pas traduit, mais corrigé la traduction de Paul d'Édesse - mais dire cela équivaut à affirmer que les manuscrits de S2 ne représentent plus fidèlement le travail de Paul d'Édesse. Ou bien, plus probablement, on peut mettre en rapport la présence dans le ms. A de notes marginales attribuées à Jacques d'Édesse avec le séjour de ce dernier au monastère de Mar Eusebona, là où précisément le ms. A a été copié plus tard en 834. Les notes marginales de ce manuscrit seraient à l'origine de la prétendue révision de Jacques d'Édesse ${ }^{33}$. En ce qui concerne la date de la première traduction, A. de Halleux est d'avis qu'elle est antérieure au schisme christologique. Il la situe plus haut dans le temps que S. Brock : il la rattache aux travaux que l'école d'Édesse a entrepris à partir du deuxième quart du $5^{\mathrm{e}} \mathrm{s} .{ }^{34}$ Cette première traduction est au mieux conservée dans le ms. T ; pourtant ce dernier représente déjà une copie légèrement révisée de l'ancienne version ${ }^{35}$. Mais l'apport majeur d'A. de Halleux a été la découverte d'une version intermédiaire $(\mathrm{Sm})^{36}$, qu'il date d'après $550^{37}$. Se fondant sur le relevé exhaustif des variantes du

\footnotetext{
${ }^{30}$ J.S. ASSEMANI, Bibliotheca Orientalis, III/1, p. 175 : Hiba alias Denha $\left(1^{\mathrm{e}}\right.$ moitié du $8^{\mathrm{e}} \mathrm{s}$.) aurait composé un commentaire des deux tomes de Grégoire.

${ }^{31}$ Elles apparaissent dans le ms. $\mathrm{F}$ et dans les commentaires $(\mathrm{Cm} 3$ fol. 100va-101ra et 102va-103ra).

32 W. LÜDTKE, «Zur Überlieferung », p. 265; A. BAUMSTARK, Geschichte, p. 251-252.

${ }^{33}$ Les notes marginales n'apparaissent pas seulement dans le ms. A ; on en lit également d'autres du même type dans les mss D $\varepsilon \eta$ (voir ci-dessous).

${ }^{34}$ Voir aussi ci-dessous les notes 66 et 67.

35 Voir A. DE HALlEUX, «L'homélie baptismale », p. 20-26. Voir les exemples donnés plus loin à la note 69 .

${ }^{36}$ A. DE HALleUX, « L’homélie baptismale », p.12-20.

${ }^{37}$ Voir ci-dessous note 66.
} 
Discours 40 (Sur le baptême) ${ }^{38}$, il est le premier à mettre en lumière l'existence d'une version moyenne entre la première version et la révision de Paul d'Édesse. Elle apparaît dans les deux autres témoins rangés jusqu'alors parmi les témoins de la version ancienne ${ }^{39}$, à savoir les manuscrits UV (les deux parties d'un homéliaire jacobite non antérieur au $6^{\mathrm{e}}-7^{\mathrm{e}} \mathrm{s}$.) et $\mathrm{W}$ (collection partielle du $9^{\mathrm{e}}-10^{\mathrm{e}}$ s.). On ajoute aujourd'hui, pour le Discours 40, les fragments conservés dans les mss Fg40a et Fg40c.

Sur les 44 Discours conservés en syriaque ${ }^{40}$, tous ne l'ont pas été dans chacune des versions. Sept ont été conservés par les trois versions, dix par les versions S1 et S2, sept par les versions $\mathrm{Sm}$ et $\mathrm{S} 2$, et vingt par la seule version S2. On notera que le Discours 35, dont l'authenticité est discutée, est absent de la tradition directe syriaque.

- S1 Sm S2: 13*, 20, 28*, 39*, 40, 41, 45

- S1 S2 : 1, 25*, 27, 29, 30, 31, 33, 34, 38*, 44

- Sm S2 : 2, 14, 19, 21, 37, 42, 43*

- S2:3, 4, 5, 6, 7, 8, 9, 10, 11, 12, 15, 16, 17, 18, 22, 23, 24, $26,32 *, 36$.

Jusqu'à présent les Discours suivants ont été édités pour le syriaque : $1,2,3,13,27,28,29,30,31,38,39,40,41^{41}$.

Pour conclure ce tour d'horizon, on soulignera combien le rôle des versions est important pour l'établissement du texte grec des Discours de Grégoire de Nazianze. Le latin, dans la traduction de Rufin, est un témoin d'un état de texte ancien capital pour la reconstitution de l'original grec, alors qu'on admettait jusqu'ici que le caractère non littéral de cette traduction la rendait inutilisable pour une comparaison textuelle. Pour se rendre compte de l'apport de Rufin, il suffit de consulter l'édition des Discours en arménien, en particulier le Discours 2 où la comparaison est constamment menée avec Rufin ${ }^{42}$. La comparaison des versions entre elles a permis de découvrir des accords textuels entre les trois versions anciennes, à savoir le latin,

\footnotetext{
${ }^{38}$ Le nombre des variantes de $\mathrm{S} 1$, pris en bloc, vis-à-vis de $\mathrm{S} 2$ est de plus de 2500. Voir A. DE HALLEUX, « L’homélie baptismale », p. 10.

${ }^{39}$ On retrouve encore cette répartition erronée, en 2000, dans A. SCHMIDT - M. QUASCHNING-KIRSCH, « Die syrischen Handschriften », p. 111.

${ }^{40}$ Parfois sous forme mutilée (le numéro du Discours est alors accompagné d'un astérisque dans le tableau qui suit).

${ }^{41}$ Le Corpus Nazianzenum contient l'édition des Discours 40 (par J.-Cl. Haelewyck en 2001), 13 et 41 (par A. Schmidt en 2002), 27, 38 et 39 (par J.Cl. Haelewyck en 2005), 28, 29, 30 et 31 (par J.-Cl. Haelewyck en 2007) et 1,2 et 3 (par J.-Cl. Haelewyck en 2011).

${ }^{42}$ Voir AUSSI C. MACÉ - M. DUBUISSON, «L’apport des traductions anciennes ».
} 
l'arménien et le syriaque, à l'encontre du grec. Ces versions sont antérieures aux plus anciens manuscrits grecs conservés et reflètent par endroits un état du texte grec perdu dans l'ensemble de la tradition grecque et qui pourrait bien avoir été le texte original de Grégoire.

\section{Une révision continuelle}

Une des caractéristiques remarquables de la tradition syriaque des Discours de Grégoire de Nazianze est l'interdépendance des versions. La version ancienne, la version moyenne et la version récente n'ont pas été réalisées indépendamment les unes des autres. Elles sont le fruit d'une activité recensionnelle continue visant à serrer de plus en plus près le texte grec. Illustrer ce processus de révision continuelle, qui rappelle celui qui a donné naissance aux versions syriaques des Évangiles, est précisément l'objectif de cet article.

Les différences entre les trois versions syriaques des oeuvres de Grégoire de Nazianze résident pour une bonne part dans le choix différent que chacun des traducteurs a opéré dans le domaine du vocabulaire. Il nous a dès lors paru utile de nous livrer à un examen du vocabulaire des versions syriaques des Discours $27,38,39$ et 40 . Le tableau qui suit ${ }^{43}$ contient les exemples les plus marquants (un peu plus de 800 mots). Ont été choisis les termes pour lesquels une différence significative apparaît dans les versions. Le relevé n'est donc pas exhaustif ${ }^{44}$. À la suite du tableau on présentera une analyse des données en nous limitant à quelques exemples illustratifs.

\begin{tabular}{|c|c|c|c|c|}
\hline Grec & $P G$ & $\mathrm{~S} 1$ & $\mathrm{Sm}$ & S2 \\
\hline$\dot{\alpha} \beta$ & 40,7 & 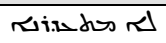 & 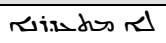 & ת \\
\hline$\dot{\alpha} \gamma \alpha \theta$ ó $\varsigma$ & 38,9 & rkant & & n \\
\hline$\dot{\alpha} \gamma \alpha \lambda \lambda i ́ \alpha \sigma 1 \varsigma$ & 40,38 & $r_{5^{0 . x}}$ & $r_{5}^{0 . x}$ & riai \\
\hline$\dot{\alpha} \gamma \alpha \lambda \lambda+\alpha ́ \omega$ & 40,25 & $10 i$ & $10 i$ & Kiv \\
\hline 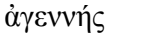 & 40,25 & $\approx m a t$ & straf & 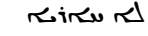 \\
\hline \multirow[t]{2}{*}{$\dot{\alpha} \gamma v i \zeta \omega$} & 38,16 & pa. فגז & & pa. iَح, \\
\hline & 40,38 & i. iص, pa & i. הحر pa & pa. \\
\hline \multirow[t]{3}{*}{$\dot{\alpha} \gamma v 0 \varepsilon \dot{\varepsilon} \omega$} & 27,5 & له ماح & & $\vec{v}$ \\
\hline & 40,10 & له ما.ح & له ה.ר & af. \\
\hline & 40,34 & ه & هم حاح & af. \\
\hline
\end{tabular}

${ }^{43}$ Les abréviations suivantes ont été employées dans le tableau : pa. = pael ; af. $=$ afel ; etpa. $=$ etpael ou etpaal ; p. $=$ participe $($ actif $) ;$ p.p. $=$ participe passif; var. = variante dans la tradition grecque ; l'absence d'indication signifie que le verbe est au peal, à l'etpeel, ou encore qu'il s'agit d'un quadrilittère aisément identifiable.

${ }^{44}$ Une étude exhaustive du vocabulaire ne pourra être menée qu'à partir d'une concordance complète des traductions syriaques des oeuvres de Grégoire. Cette concordance est en cours de réalisation au Centre de Traitement Automatisé des Langues (CENTAL) de l'Université Catholique de Louvain. Nous ajouterons de temps à autre des exemples tirés du Discours 1 (S1 et S2), car ce dernier a déjà fait l'objet d'une lemmatisation et d'un alignement du grec et du syriaque. 


\begin{tabular}{|c|c|c|c|c|}
\hline Grec & $P G$ & $\mathrm{~S} 1$ & $\mathrm{Sm}$ & $\mathrm{S} 2$ \\
\hline 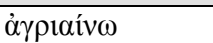 & 27,7 & iivadir & & ححiّة حصi. \\
\hline$\alpha ̋ \gamma \chi \omega$ & 27,5 & حله & & ard \\
\hline 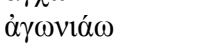 & 38,15 & sdidir & & sition \\
\hline $\begin{array}{l}\dot{\alpha} \delta 1 \alpha i ́ \rho \varepsilon \tau \sigma \varsigma \\
(-\tau \omega \varsigma)\end{array}$ & 39,11 & 幽 & & 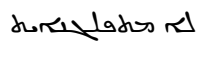 \\
\hline$\alpha \delta$ ¿кí & 40,11 & rhis & rhi & rhatar \\
\hline 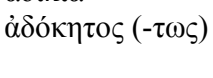 & 40,46 & 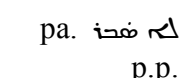 & pa.p.p. ه ف & هم علد \\
\hline$\dot{\alpha} \varepsilon i ́ \delta \omega$ & 27,5 & pa. isi & & קa. קרu \\
\hline 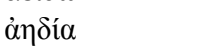 & 27,2 & Rhainf & & rharem a \\
\hline$\alpha \theta \varepsilon \rho \alpha ́ \pi \varepsilon v \tau о \varsigma$ & 39,15 & risludus a & תم & reorstas a \\
\hline$\alpha \theta \lambda \eta \eta \iota^{\prime}$ & 27,2 & readads & & rhacoldir \\
\hline áî́os & 38,8 & $\begin{array}{c}\text { padus ps } \\
\text { mudur }\end{array}$ & & resados \\
\hline$\alpha i \mu \alpha \tau \omega ́ \delta \eta \varsigma$ & 39,20 & $\begin{array}{r}\text { خdo ح.sa. } \\
\text { par }\end{array}$ & 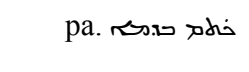 & rasis \\
\hline 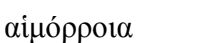 & 40,33 & זוג.א הותמה & וזים הגדתה & iז.s dis \\
\hline dî $\rho \omega$ & 27,5 & خلر. pa & & af. \\
\hline$\alpha i ̌ \sigma \theta \eta \sigma ı s$ & 38,11 & Re i & & Rheli \\
\hline 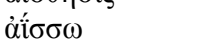 & 40,30 & jowotir & tri & 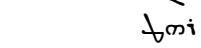 \\
\hline 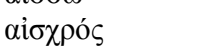 & 39,3 & pa.p.p. غحi & & Rine \\
\hline$\alpha i \sigma \chi v ́ v \eta$ & 40,27 & रमs soud & Rhidima/rh_ssud & rhidins \\
\hline$\alpha i \tau i \alpha ́ o \mu \alpha \imath$ & 40,20 & حal & حسי & rexi \\
\hline 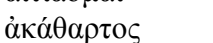 & 40,35 & لهم ה.רא & لهم וגטم & rail \\
\hline 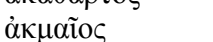 & 40,37 & stules & حחוr & RhaL \\
\hline 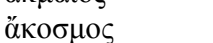 & 39,1 & & له ד כגי & اه حسת \\
\hline 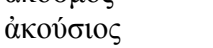 & 40,23 & 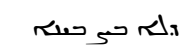 & 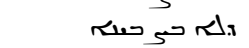 & له \\
\hline$\dot{\alpha} \kappa о \nu \tau i ́ \zeta \omega$ & 40,38 & af. & af. & onsiditis \\
\hline 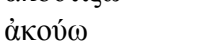 & 40,16 & عدs & عs & riodir \\
\hline 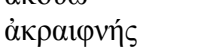 & 40,37 & Rivir & ז.רא & ק.ר מת p.a \\
\hline$\alpha \lambda \alpha \zeta o v \varepsilon i ́ \alpha$ & 27,10 & Rhasi & & Rרמשim \\
\hline$\dot{\alpha} \lambda \gamma \varepsilon ı v o ́ \varsigma$ & 40,41 & حمد p.a. & מתח & דתחדה \\
\hline$\dot{\alpha} \lambda \eta \theta \hat{\eta} \varsigma(-\tilde{\omega} \varsigma)$ & 39,12 & Ritr.s & مrive & huritiv \\
\hline 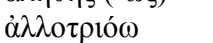 & 39,12 & גمriح גor & טنi, & حشi, \\
\hline 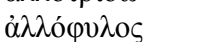 & 40,43 & radara & radela & Rhoir iur \\
\hline \multirow[t]{2}{*}{$\alpha \lambda$ ó $\gamma 1 \sigma \tau \mathrm{\alpha}, \varsigma$} & 40,28 & rals & r & Rharus \\
\hline & 40,45 & ه هله & له هله & נים כمس ה \\
\hline 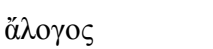 & 38,17 & ه ه דلel & & ת. \\
\hline$\dot{\alpha} \mu \alpha \theta \hat{n} \varsigma$ & 27,9 & م م م rad & & afo \\
\hline$\dot{\alpha} \mu \alpha \dot{\alpha} \tau \eta \mu \alpha$ & 40,31 & रत्చv & रतڤ & Rhuto \\
\hline$\dot{\alpha} \mu \alpha \rho \tau i ́ \alpha$ & 40,39 & रत्冋, & reto & rheto \\
\hline$\dot{\alpha} \mu \beta \lambda v \omega \pi i \alpha$ & 40,45 & 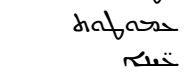 & 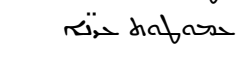 & Rhaim \\
\hline $\alpha \mu \varepsilon^{\prime} v \omega \nu$ & 39,8 & rivar & Riear & ومس \\
\hline$\dot{\alpha} \mu \varepsilon \tau \rho i ́ \alpha$ & 27,4 & Rhwars a & & R Raness \\
\hline$\alpha \mu о \rho \rho о \varsigma$ & 40,34 & p.p. $\pi \infty 0$ & rhasis $\nabla_{x}$ & rhasx rux \\
\hline \multirow[t]{2}{*}{ 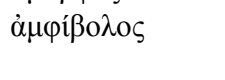 } & 39,19 & 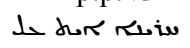 & 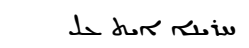 & etpa. ratir \\
\hline & 40,11 & p. undiadir & p. undiadir & pa.p.p. فru \\
\hline$\dot{\alpha} v \alpha \beta 0 \lambda \eta \dot{n}$ & 40,20 & sticustidas & rhaumbidas & रrmad \\
\hline 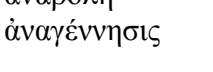 & 40,44 & 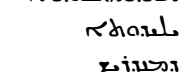 & 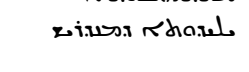 & 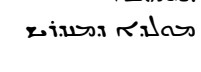 \\
\hline 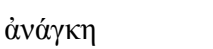 & 40,12 & مسلمת & אחسم & مa \\
\hline$\alpha \alpha^{\prime} \gamma \omega$ & 40,14 & Ris & rii & arb \\
\hline \multirow[t]{2}{*}{$\dot{\alpha} v \alpha \gamma \omega \gamma \eta \dot{ }$} & 39,2 & & Atraax & Rhores \\
\hline & 40,36 & خلد.pa.p.p & aldara & Rhinda \\
\hline$\alpha \dot{\alpha} \alpha \dot{\alpha} \delta 0 \sigma 1 \varsigma$ & 27,9 & rhasas & & נרגה \\
\hline 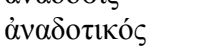 & 40,39 & فلح pa.p.p. & p. ind dir & witio das \\
\hline$\alpha \dot{\alpha} \alpha 1 \delta \varepsilon i ́ \alpha$ & 39,2 & & 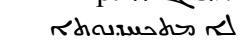 & rhasisan \\
\hline 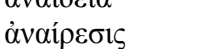 & 40,11 & 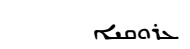 & 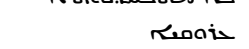 & rif \\
\hline$\dot{\alpha} \nu \alpha \iota \varepsilon \dot{\varepsilon} \omega(-о \mu \alpha \imath)$ & 40,32 & coididir & coididir & this \\
\hline$\alpha \alpha_{\alpha i ́} \sigma \theta \eta \tau \varsigma \varsigma$ & 39,6 & whrov is & whrolia & ๘am \\
\hline$(-\tau \omega \varsigma)$ & 40,28 & Rhry i $\sigma \mathrm{s}$ & rodry i $\sigma \mathrm{x}$ & ג䧄 \\
\hline$\alpha v \alpha \theta \alpha ́ \lambda \lambda \omega$ & 40,33 & 10i & 10i & 10i y9m \\
\hline 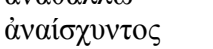 & 40,35 & هivedos & sinerdos $ه$ & 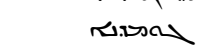 \\
\hline$\alpha v \alpha \kappa \alpha \lambda \dot{\varepsilon} \omega$ & 40,31 & 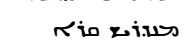 & Ritus & 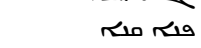 \\
\hline
\end{tabular}




\begin{tabular}{|c|c|c|c|c|}
\hline Grec & $P G$ & $\mathrm{~S} 1$ & $\mathrm{Sm}$ & $\mathrm{S} 2$ \\
\hline 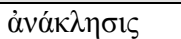 & 40,9 & Kגi & مגit & rhareadids \\
\hline$\dot{\alpha} v \alpha \lambda \alpha \mu \beta \dot{\alpha} v \omega$ & 39,2 & & 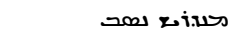 & عمل \\
\hline \multirow[t]{3}{*}{ 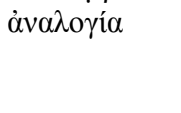 } & 27,8 & Rhossul & & Nosug \\
\hline & 40,45 & Rhosul & rhasul & Rhuars \\
\hline & 40,45 & rhasen & rhasul & rhwars \\
\hline '̊vó $\mu \nu \eta \sigma ı \varsigma$ & 27,10 & كxmon & & Rhousmas \\
\hline Q́v $\alpha v \delta \rho \circ \varsigma$ & 27,9 & لم حصir & & rax \\
\hline \multirow[t]{3}{*}{ 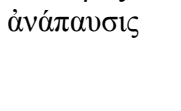 } & 27,8 & res & & Rhues \\
\hline & 40,33 & rew & ren & حمess \\
\hline & 40,35 & rue & rees & Rhues \\
\hline 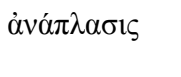 & 40,2 & 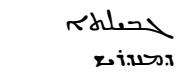 & 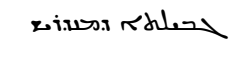 & 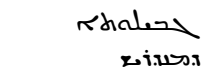 \\
\hline 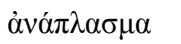 & 39,3 & & حaler & קרחיםr \\
\hline 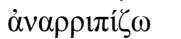 & 27,5 & pa. نحص & & Jial \\
\hline öv $\alpha \rho \chi O \varsigma$ & 40,45 & wior $\Delta x$ & rine & 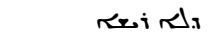 \\
\hline$\dot{\alpha} v \alpha \sigma \tau \varepsilon \dot{\varepsilon} \lambda \lambda \omega$ & 40,40 & pa. ل山 & pa. J山 & pa. paم \\
\hline$\dot{\alpha} v \alpha \tau \varepsilon ́ \lambda \lambda \omega$ & 40,36 & af. & זת & זנת \\
\hline$\dot{\alpha} v \alpha \chi \omega v \varepsilon v ́ \omega$ & 40,8 & 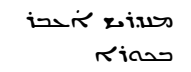 & 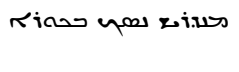 & 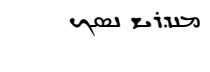 \\
\hline$\dot{\alpha} v \alpha \psi v ́ \chi \omega$ & 40,38 & pa. ف. & pa. فيخ & קהט \\
\hline 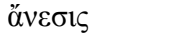 & 39,18 & سيك & عمحملم & عمحملم \\
\hline 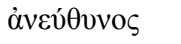 & 39,7 & 几d & pa.p. pa ندר & pa.p. pم نعد \\
\hline$\dot{\alpha} v \varepsilon v \lambda \alpha \beta \tilde{\eta} \varsigma$ & 40,16 & 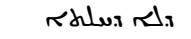 & $\sigma_{\text {i.ear }} \sigma_{x}$ & هم حسרת \\
\hline$\alpha \dot{\alpha} \eta \dot{\rho} \rho$ & 38,13 & אתושא & & rin \\
\hline$\dot{\alpha} v \theta \dot{\varepsilon} \lambda \kappa \omega$ & 40,26 & etpa. „لגidir & etpa. „لsidir & pa. خan \\
\hline d’vió $\tau \rho \varepsilon v \tau o \zeta$ & 39,18 & रमneor & rhaneor & Rhamods \\
\hline \multirow[t]{4}{*}{ óvoro } & 27,9 & rdialoo & & 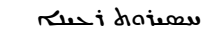 \\
\hline & 39,6 & سمा & سمam & \\
\hline & 39,7 & ๘am & Rhowe & \\
\hline & 40,20 & rhevid R & محلم & ram daieas \\
\hline òvouía & 40,45 & rroi & Rrai & Rhomoss \\
\hline 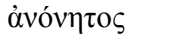 & 40,27 & ridias & ه & idios $\sim x$ \\
\hline$\dot{\alpha} v \tau \imath \delta \dot{\delta} \delta \omega \mu \imath$ & 27,7 & pa.pa & & دمد vلa \\
\hline$\dot{\alpha} v \tau i ́ \theta \varepsilon \tau o \varsigma$ & 40,37 & סمסד & סمסק סمחحلה ג & مممحليك \\
\hline 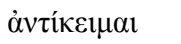 & 39,2 & & הلممحله & مممحلיم \\
\hline 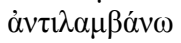 & 40,40 & حa a d & حر a da & חתר נחב \\
\hline$\grave{\alpha} v \tau 1 \mu \varepsilon \tau \rho \varepsilon \dot{\varepsilon} \omega$ & 40,9 & $\begin{array}{r}\text { af. } \\
\text { and }\end{array}$ & af. & محصل hal \\
\hline$\dot{\alpha} \nu \tau 1 \sigma \omega \dot{\zeta} \omega$ & 40,9 & ,ater uam & ,aber ugm & hoal, atrer \\
\hline$\dot{\alpha} v \tau \imath \tau i ́ \theta \eta \mu \imath$ & 40,10 & 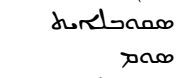 & مممحلهمان صمج & סمק لممحل \\
\hline$\dot{\alpha} \nu \tau 1 \tau \rho \varepsilon ́ \chi \omega$ & 40,25 & 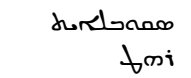 & דآ & 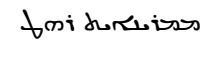 \\
\hline$\dot{\alpha} v \omega \mu \alpha \lambda i ́ \alpha$ & 40,34 & م R Rasuls & rhane & Rhane \\
\hline$\dot{\alpha} v \omega \dot{\mu} \mu \alpha \lambda \circ \varsigma$ & 40,41 & 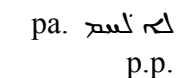 & pa.p.p. لهم لبح & 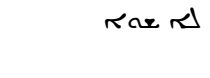 \\
\hline \multirow[t]{5}{*}{ 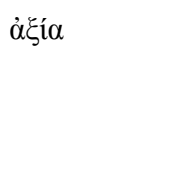 } & 39,14 & אمחiم & אمחוiא & عمله \\
\hline & 40,6 & אחمוin & אممוiא & rifar \\
\hline & 40,26 & Kh⿻ir & אمחمir & عaلr \\
\hline & & مאم:iم & & \\
\hline & 40,42 & Rم:R & אمסiא & rifar \\
\hline 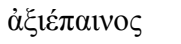 & 38,10 & ren ren & & p. عم لمملهم \\
\hline$\alpha \not \alpha \xi 10 \varsigma(-\omega \varsigma)$ & 39,19 & dorear & אمب זוix & 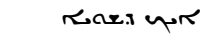 \\
\hline 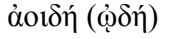 & 27,5 & Khatesi & & Khwared \\
\hline 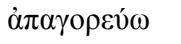 & 40,27 & genم & s & etpa. afفidi \\
\hline$(-o \mu \alpha \imath)$ & 38,12 & مodir & & rdir \\
\hline \multirow[t]{2}{*}{$\alpha \pi \alpha \lambda \lambda \alpha \gamma \eta ́$} & 40,24 & פملעr & त丸क्त & रגक人 \\
\hline & 40,32 & $\sigma_{5}$ & rhace & 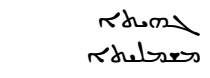 \\
\hline$\dot{\alpha} \pi \alpha \lambda \lambda \alpha \dot{\alpha} \sigma \sigma \omega$ & 40,20 & pa. ف5 & ف5ر, pa. & af. \\
\hline$\dot{\alpha} \pi \alpha \lambda o^{\prime} \varsigma$ & 38,12 & स.89 & & iٓحسת \\
\hline$\alpha \dot{\alpha} \alpha \dot{\alpha} v \theta \rho \omega \pi \varsigma$ & 40,14 & 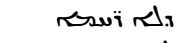 & 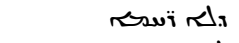 & Noviv \\
\hline \multirow[t]{2}{*}{$\alpha \pi \alpha \xi$ ı́́ } & 40,27 & rar $\alpha$ & 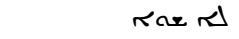 & is \\
\hline & 70,27 & ه & 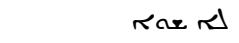 & ist \\
\hline
\end{tabular}




\begin{tabular}{|c|c|c|c|c|}
\hline Grec & $P G$ & $\mathrm{~S} 1$ & $\mathrm{Sm}$ & S2 \\
\hline$\dot{\alpha} \pi \alpha \rho \alpha i ́ \tau \eta \tau \varsigma$ & 40,14 & caldas $\sim \Delta x$ & $J$ זhers rax & 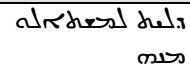 \\
\hline 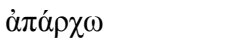 & 40,2 & pa. , iغ & pa. , i亡 & Rhuri Ram \\
\hline 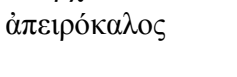 & 40,29 & $\begin{array}{l}\text { Sols } \\
\text { rint }\end{array}$ & 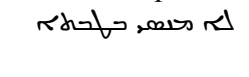 & 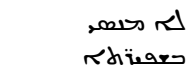 \\
\hline 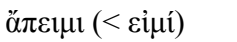 & 40,25 & هم مiٔحصح & لهم مiٔحسم & Ravi \\
\hline$\dot{\alpha} \pi \varepsilon \imath \rho i ́ \alpha(<\pi \varepsilon \tilde{\imath} \rho \alpha)$ & 40,20 & פחתר & Rhowass & Rhanears \\
\hline 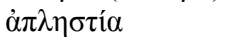 & 27,7 & rharals & & rhas \\
\hline$\not \alpha \pi \lambda \eta \sigma \tau о \varsigma$ & 40,10 & حلمحه & حلarrr & هي \\
\hline$\dot{\alpha} \pi \hat{\theta} \theta \varepsilon \sigma 1 \varsigma$ & 40,3 & Now & N5eo & reule \\
\hline 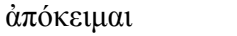 & 27,8 & (סח & & 论 \\
\hline$\dot{\alpha} \pi$ & 27,2 & doutwotir & & $\alpha \pi$ \\
\hline \multirow[t]{2}{*}{ 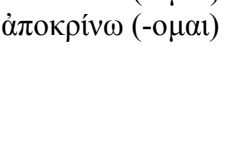 } & 27,8 & בגin & & فر pa. \\
\hline & 40,28 & 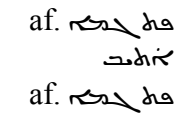 & af. & فلر pa. ف \\
\hline$\dot{\alpha} \pi \circ \kappa \tau \dot{\alpha} \alpha \mu \alpha \iota$ & 40,32 & 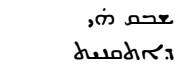 & duadira, & etpa. sition \\
\hline$\alpha \dot{\alpha} \pi \mathrm{ov \varepsilon ́} \mu \omega$ & 39,19 & pa. فi & pa. فi & מחב \\
\hline 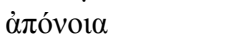 & 39,18 & rhioders & Shades & rhatars \\
\hline 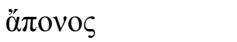 & 40,9 & 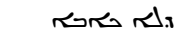 & 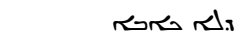 & x.له حدله \\
\hline$\dot{\alpha} \pi \circ \pi \varepsilon \dot{\varepsilon} \mu \pi \omega$ & 40,45 & r.x & r.x & i \\
\hline 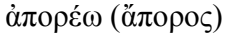 & 40,34 & ines & ines & $n$ 计 $5^{2}$ \\
\hline 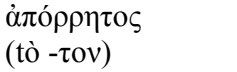 & 40,20 & ritr & rhaneas & حمسمוhr \\
\hline 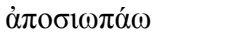 & 40,10 & علم & عله & othe \\
\hline 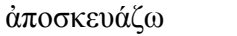 & 27,7 & itroo & & الم \\
\hline$\dot{\alpha} \pi 0 \sigma \tau \alpha \tau \imath \kappa o ́ \varsigma$ & 38,9 & rovitos & & Rxaiss \\
\hline$\dot{\alpha} \pi$ о $\tau \dot{\theta} \theta \eta \mu \iota$ & 40,32 & r.x & R. R & סחד ק \\
\hline \multirow{2}{*}{ 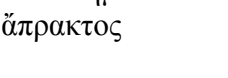 } & 40,35 & 西 & له & حeter \\
\hline & 40,35 & هת حרז: & נه حרו: & xhainso \\
\hline$\dot{\alpha} \pi \rho \circ \sigma \varepsilon \xi \hat{i} \alpha$ & 40,45 & هم ساحك & ه N & Rhainm \\
\hline$\ddot{\alpha} \pi \tau \omega$ & 40,33 & 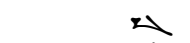 & $z$ & ard \\
\hline 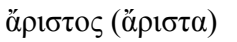 & 40,35 & hrobit & duroit & i \\
\hline 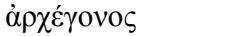 & 40,5 & 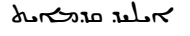 & 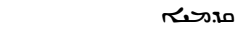 & مאודה م \\
\hline \multirow[t]{2}{*}{$\dot{\alpha} \rho \chi \eta\left(\dot{\alpha} \pi^{\prime} \dot{\alpha} \rho \chi \tilde{\eta} \varsigma\right)$} & 39,1 & & דק حich & rine \\
\hline & 40,7 & Rti & red & rine \\
\hline$(\dot{\varepsilon} \xi \dot{\alpha} \rho \chi \tilde{\eta} \varsigma)$ & 40,44 & duris & duris & rinc \\
\hline ó $\rho \rho \eta \tau о \varsigma(-\tau \omega \varsigma)$ & 40,45 & 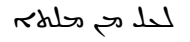 & 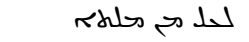 & 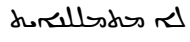 \\
\hline$\dot{\alpha} \rho \chi \imath \tau \varepsilon ́ \kappa \tau \omega \nu$ & 40,43 & 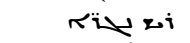 & Riv & مiind \\
\hline \multirow[t]{2}{*}{$\dot{\alpha} \sigma \dot{\varepsilon} \beta \varepsilon 1 \alpha$} & 27,7 & rhalar & & Rrai \\
\hline & $\begin{array}{l}27,9 \\
\text { (var.) }\end{array}$ & حمه & & Reai \\
\hline 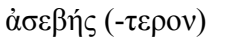 & 27,7 & 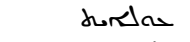 & & dorueitiod \\
\hline$\dot{\alpha} \sigma \varepsilon \dot{\lambda} \lambda \gamma \varepsilon 1 \alpha$ & 40,37 & rhangl & rhangl & Rhatue \\
\hline ắ $\sigma \mu \alpha$ & 40,3 & Rdiusi & Rhiss & Rhwaned \\
\hline 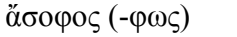 & 27,9 & ד & & גas \\
\hline \multirow[t]{2}{*}{$\dot{\alpha} \sigma \pi i \zeta$} & 40,10 & rois & roin & conor \\
\hline & 40,34 & roil & mano & manr \\
\hline 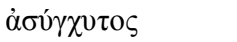 & 39,12 & זים حملحله & هم حلفله & الم حلفله \\
\hline $\begin{array}{l}\dot{\alpha} \sigma 0 \lambda \lambda \hat{\gamma} \gamma 1 \sigma \tau \mathrm{\sigma} \\
(-\omega \varsigma)\end{array}$ & 40,21 & rharus a & & x. له هم تله \\
\hline$\dot{\alpha} \sigma \varphi \alpha ́ \lambda \varepsilon 1 \alpha$ & 40,35 & rhar,s & rhan, & عملحسك \\
\hline$\dot{\alpha} \sigma \varphi \alpha \lambda \hat{\zeta} \omega \omega$ & 40,15 & 艺 & it & ז'וm \\
\hline$\dot{\alpha} \sigma \varphi \rho \alpha ́ \gamma 1 \sigma \tau \sigma \varsigma$ & 40,28 & 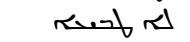 & rald & هم שגss \\
\hline$\dot{\alpha} \sigma \chi \eta \dot{\mu} \mu \nu$ & 40,40 & wroor & जsor & reoing \\
\hline$\dot{\alpha} \sigma \chi 0 \lambda \dot{i} \alpha$ & 40,18 & Rhaims & حسلم & حسلم \\
\hline$\dot{\alpha} \sigma \omega ́ \mu \alpha \tau \sigma \varsigma$ & 39,19 & ג & 䧑 & ه \\
\hline 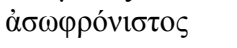 & 39,18 & ק. & axiss $\sim x$ & هم דحمسك \\
\hline$\dot{\alpha} \tau \varepsilon \dot{\lambda} \overline{\varepsilon \sigma \tau o \varsigma}$ & 40,28 & ris & ه & هم תעמרلה \\
\hline
\end{tabular}

${ }^{45}$ Une note marginale signale la variante rhaimm. 


\begin{tabular}{|c|c|c|c|c|}
\hline Grec & $P G$ & S1 & $\mathrm{Sm}$ & S2 \\
\hline \multirow[t]{3}{*}{$\grave{\alpha} \tau \mu \mu \alpha \dot{\zeta} \omega \omega$} & 27,4 & pa. i & & $t_{a x}$ \\
\hline & 27,5 & pa. ند & & $f_{a}$ \\
\hline & 39,16 & pa. is & pa. i i & tar \\
\hline \multirow[t]{2}{*}{$(-o \mu \alpha \imath)$} & 39,6 & etpa. if ${ }^{2}$ & etpa. $i_{5}$ & Verdidir \\
\hline & 39,7 & etpa. if & etpa. if s $^{r}$ & terditir \\
\hline 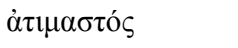 & 40,40 & R & 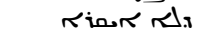 & p. terdidir \\
\hline 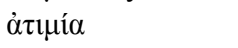 & 39,13 & Rivs & ri 5 & Rhater \\
\hline$\ddot{\alpha} \tau \mu \rho \varsigma$ & 40,9 & pa.p.p. ن خد & pa. p.p. ند & p.p. \\
\hline 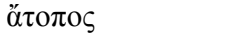 & 27,1 & pa.p.p. & & عصiv \\
\hline ö $\tau \rho \varepsilon \pi \tau о \varsigma$ & 39,13 & randos & rialudurs & rialudurs a \\
\hline$\dot{\alpha} \tau \hat{\chi} \chi \eta \mu \alpha$ & 39,5 & & rhas diares & rhowax \\
\hline$\alpha \cup ่ \gamma \alpha ́ \zeta \omega$ & 40,5 & pa. ن im & pa. ims & af. \\
\hline$(-o \mu \alpha \imath)$ & 40,34 & $\begin{array}{l}\text { etpa. } \\
\text { ets }\end{array}$ & etpa. & Nitidir \\
\hline$\alpha \cup ̉ \gamma \eta ́$ & 39,20 & الفمr & الفمك & الح \\
\hline öü̇̀ & 38,9 & هam & & ما ه \\
\hline$\alpha u ́ \rho \alpha$ & 27,5 & Ruai & & תחשרא \\
\hline \multirow{2}{*}{ 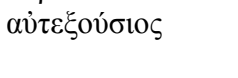 } & 38,12 & rho diafless & & row diafless \\
\hline & 38,12 & 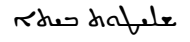 & & rhe dafles \\
\hline 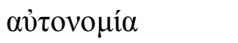 & 39,7 & 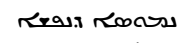 & reaix rooss & reas dicooss \\
\hline$\dot{\alpha} \varphi \alpha i ́ \rho \varepsilon \mu \alpha$ & 40,40 & عمله & عمله & Rein \\
\hline$\dot{\alpha} \varphi \alpha \imath \rho \varepsilon ́(-о \mu \alpha \imath)$ & 40,16 & Johere & Joher & 山 dir \\
\hline$\dot{\alpha} \varphi \alpha v i \sigma \tau \imath \kappa o ́ \varsigma$ & 40,36 & كטרلה & תטבلنه & raf \\
\hline$\dot{\alpha} \varphi v ́ \lambda \alpha \kappa \tau \sigma \varsigma$ & 40,16 & pa. imi & 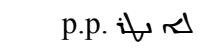 & 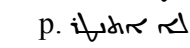 \\
\hline 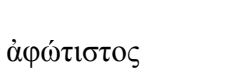 & 40,34 & 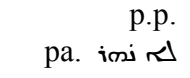 & pa.p.p. له نهi & Rimas \\
\hline \multirow{2}{*}{ 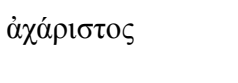 } & 40,34 & p.p. & ه & ת \\
\hline & 40,38 & 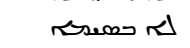 & هم حمs & 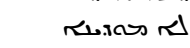 \\
\hline 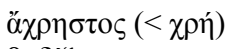 & 27,10 & Rhureu $\Delta x$ & & anev $\Delta$. \\
\hline$\beta \alpha \delta i \zeta \omega$ & 40,27 & pa. $n$ गं & pa. चगं & S \\
\hline$\beta \alpha ́ \delta 1 \sigma \mu \alpha$ & 27,52 & חדרת & & rhalm \\
\hline$\beta \alpha \theta v ́ s$ & 40,17 & معiنم & معiخم & حدنمك \\
\hline$\beta \alpha ́ \lambda \lambda \omega$ & 40,39 & R.x & R.x & si \\
\hline \multirow{4}{*}{$\beta \alpha ́ \pi \tau \imath \sigma \mu \alpha$} & 39,17 & Rho:oss & حתוגוr & حמה.r \\
\hline & 40,4 & rab & rhuinsas & Rh.xoss \\
\hline & 40,22 & Rhoxoss & rhuinsas & حמהות \\
\hline & 40,22 & Rhonoss & Rhoross & حתות \\
\hline \multirow[t]{2}{*}{ 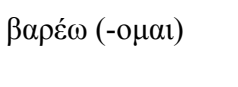 } & 40,33 & مi & in & etpa. حdidir \\
\hline & 40,19 & ما & مi & etpa. حdidir \\
\hline$\beta \alpha \tau o ́ \varsigma$ & 27,8 & rixds & & rhioulestas \\
\hline$\beta \delta \varepsilon \lambda v \kappa \tau o ́ \varsigma$ & 39,6 & rast & rast & af. \\
\hline 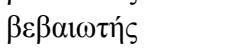 & 27,1 & תדمימתי & & ritivs \\
\hline$\beta 1 \alpha ́ \zeta \omega$ & 38,10 & 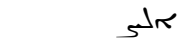 & & حكs \\
\hline$\beta \iota \alpha \sigma \tau \eta \dot{\zeta}$ & 40,24 & حוa & טוمא & مه \\
\hline \multirow{2}{*}{ Bíos } & 40,3 & نَّ & نس & 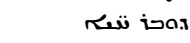 \\
\hline & 40,16 & Risax & تنه & تنسك \\
\hline 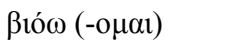 & 40,24 & p.p. סحن & p.p. & etpa. אוּ \\
\hline$\beta \lambda \dot{\alpha} \pi \tau \omega$ & 27,3 & pa. & & af. iอง \\
\hline \multirow[t]{2}{*}{ 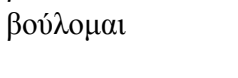 } & 39,19 & ת & ת & حת \\
\hline & 40,23 & ס & حه & S כת \\
\hline$\beta \rho \alpha \beta \varepsilon \tilde{i} о \nu$ & 40,39 & Ring & Ring & حلeله \\
\hline$\beta \rho \alpha \delta v ́ \varsigma$ & 40,25 & Rivas & rivas & ruts \\
\hline 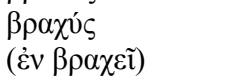 & 40,2 & Rin & Rin & ملeled \\
\hline$\gamma \alpha \mu \lambda^{\prime} \lambda 10 \varsigma$ & 40,1 & 办an & 舟 & rhabers \\
\hline 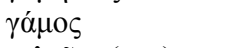 & 40,18 & 幽001 & Rhaters & om. \\
\hline 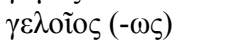 & 38,15 & ג几Rר dos & & 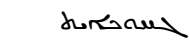 \\
\hline 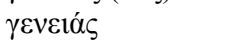 & 39,14 & דمكת & הمكح & rhvores \\
\hline$\gamma \varepsilon v v \varepsilon \dot{\tau} \omega \rho$ & 40,26 & אתמא & אתר & r.tas \\
\hline$\gamma \varepsilon ́ v \vee \eta \sigma 1 \zeta$ & 27,6 & ملح & & rharels \\
\hline$\gamma \varepsilon \omega \mu \varepsilon \dot{\tau} \tau \eta \varsigma$ & 39,14 & matrsares & matsans & 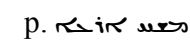 \\
\hline$\gamma \varepsilon \omega \rho \gamma o ́ \varsigma$ & 38,12 & rula & & riar \\
\hline 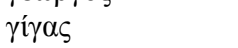 & 27,9 & (ע) & & rin \\
\hline$\gamma \lambda \omega \sigma \sigma \alpha \lambda \gamma i ́ \alpha$ & 27,7 & Sid dicrs & & 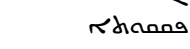 \\
\hline
\end{tabular}




\begin{tabular}{|c|c|c|c|c|}
\hline Grec & $P G$ & S1 & $\mathrm{Sm}$ & S2 \\
\hline$\gamma v \eta \sigma i o ́ \tau \eta \varsigma$ & 40,27 & Rhaizir & Rhaivir & טרברחאיא \\
\hline$\gamma \nu \omega ́ \mu \eta$ & 40,32 & عمحملم & عمحملم & 5 حله \\
\hline 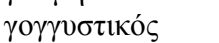 & 40,20 & saturas & תathat & $\overrightarrow{x i b x \rightarrow ~}$ \\
\hline$\gamma \rho \alpha ́ \mu \mu \alpha$ & 40,12 & rdir & rdir & مרdo \\
\hline 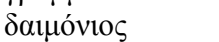 & 27,5 & Ra.x & & אחות \\
\hline$\delta \alpha i \mu \omega v$ & 38,15 & 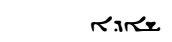 & & ro.x \\
\hline$\delta \varepsilon 1 \lambda i ́ a$ & 39,9 & rolur & הalour & Rhoublaw \\
\hline \multirow[t]{2}{*}{$\delta \varepsilon 1 \sigma 1 \delta \alpha i ́ \mu \omega v$} & 39,8 & hares & Rhacos dust & היטلג تعمות \\
\hline & & rhacos & & \\
\hline 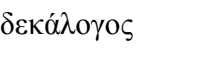 & 40,45 & 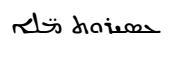 & 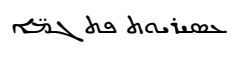 & 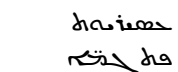 \\
\hline \multirow[t]{2}{*}{$\delta \varepsilon \lambda \varepsilon \alpha \dot{\alpha} \zeta \omega$} & 39,13 & af. & غxل & pa. \\
\hline & 39,13 & at & om. & etpa. Jintis \\
\hline \multirow[t]{2}{*}{$\delta \varepsilon \sigma \mu o ́ \varsigma$} & 40,3 & Ring & gin & rinor \\
\hline & 40,33 & rinor & rinor & Ring \\
\hline$\delta \dot{\varepsilon} \omega([\delta \eta \dot{\sigma} \sigma \omega])$ & 27,2 & ino & & م \\
\hline \multirow{5}{*}{$\begin{array}{l}\delta \varepsilon ́ \omega \\
(-o \mu \alpha 1,[\delta \varepsilon \eta ́ \sigma \omega])\end{array}$} & 38,13 & ratir & & 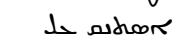 \\
\hline & 39,12 & p. In p & p. In & p. هa \\
\hline & 40,14 & مRisi & مisisi & 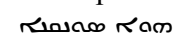 \\
\hline & 40,29 & 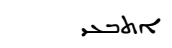 & 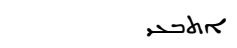 & مיمת \\
\hline & 40,46 & حه & حه & etpa.aridir \\
\hline$\delta \eta \lambda \eta \tau \eta \dot{\rho} \rho \varsigma^{2}$ & 40,14 & مه ده & مهله & مחס Ras \\
\hline$\delta \tilde{\eta} \mu \varsigma_{\varsigma}$ & 38,8 & טעחזה & & حוd \\
\hline$\delta \eta \mu 100 \rho \gamma \varepsilon \dot{\varepsilon} \omega$ & 27,9 & רבו: & & حí \\
\hline \multirow[t]{2}{*}{$\delta \eta \mu о \sigma 1 \varepsilon v ́ \omega$} & 27,2 & مזיד حل مهע & & لعم حلي نه, \\
\hline & & حרו: & & pa. \\
\hline$\delta 1 \alpha \beta \alpha i ́ v \omega$ & 27,3 & حصi & & منحل af \\
\hline$\delta 1 \alpha i ́ \rho \varepsilon \sigma 1 \varsigma$ & $39,11^{46}$ & Rxing & Rring & 幽 \\
\hline$\delta i ́ \alpha \imath \tau \alpha$ & 27,5 & Rinox & & గ290m \\
\hline 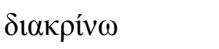 & 40,26 & leatir & leadir & etpa. yegtix \\
\hline \multirow[t]{2}{*}{$\delta 1 \alpha \lambda \varepsilon_{\varepsilon} \gamma \omega(-o \mu \alpha \imath)$} & 40,19 & خهلl & pa. خهلl & al ration \\
\hline & 40,19 & $\begin{array}{r}\text { pa. } \\
\text { pa. هـ له له }\end{array}$ & هـ & $\begin{array}{l}\text { etpa. } \\
\text { a sation }\end{array}$ \\
\hline \multirow{2}{*}{$\delta 1 \alpha \lambda \varepsilon \kappa \tau \imath \kappa o ́ \varsigma$} & 27,8 & x.بلم|eمr & & $\begin{array}{r}\text { etpa. } \\
\text { <íms }\end{array}$ \\
\hline & 27,9 & sلs & & Rimss \\
\hline \multirow{2}{*}{$\begin{array}{l}\delta 1 \alpha \dot{\alpha} \mu \varepsilon \tau \rho \circ \varsigma \\
(\dot{\varepsilon} \kappa-\rho \circ v)\end{array}$} & 39,11 & 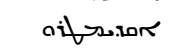 & 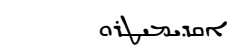 & dowar \\
\hline & & & & Rhwars \\
\hline \multirow{4}{*}{$\begin{array}{l}\delta 1 \alpha v o ́ \eta \mu \alpha \\
\delta 1 \alpha \pi \tau v ́ \omega\end{array}$} & 40,39 & Rharus & Rhewamtos & Rhenith \\
\hline & 39,3 & & i iٔم & af. منملر \\
\hline & 40,27 & תم & حم) & tar \\
\hline & 40,41 & i oi & i o & af. אזיל \\
\hline 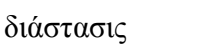 & 40,7 & Rring & Rring & rhareighss \\
\hline$\delta \dot{ }^{\prime} \sigma \tau \eta \mu \alpha$ & 38,8 & riat & & rudus \\
\hline$\delta 1 \alpha \varphi \varepsilon \rho v^{\prime} \tau \omega \varsigma$ & 27,8 & i & & duridus \\
\hline$\delta 1 \alpha \varphi \varepsilon v ́ \gamma \omega$ & 40,16 & حit & حiٔم & אתדה. \\
\hline$\delta 1 \alpha \chi \varepsilon ́ \omega$ & 39,9 & אשו. & pa. فلح & pa. فلح \\
\hline 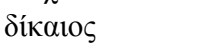 & 40,31 & 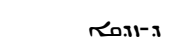 & 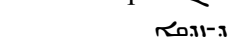 & Rhoura-s \\
\hline$\delta 1 \varepsilon \xi \dot{\varepsilon} \rho \chi 0 \mu \alpha 1$ & 40,22 & Sa & دمم & حרi \\
\hline 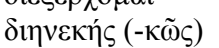 & 27,4 & حلrs & & 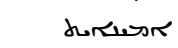 \\
\hline$\delta 101 \kappa \varepsilon ́ \omega(-о \mu \alpha \imath)$ & 40,45 & etpa. & etpa. & mintir \\
\hline$\delta 10 \chi \lambda \varepsilon^{\prime} \omega$ & 27,2 & Ri & & Rhovid is \\
\hline \multirow[t]{2}{*}{$\delta 1 \sigma \sigma o ́ \varsigma$} & 40,8 & लأid & م) & Ra.a \\
\hline & 40,8 & ruid & rid & Ra.a \\
\hline$\delta \iota \chi о \tau о \mu \varepsilon ́ \omega$ & 39,15 & 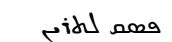 & معم مeld & a \\
\hline \multirow[t]{2}{*}{$\delta 1 \omega \gamma \mu o ́ s$} & 39,7 & 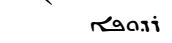 & 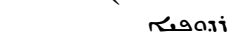 & R.9oxi \\
\hline & 40,35 & reani & Ragaii & Rhoa.ii \\
\hline
\end{tabular}

${ }^{46}$ On se gardera bien de systématiser : deux lignes plus loin S1 Sm et S2

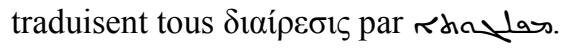




\begin{tabular}{|c|c|c|c|c|}
\hline Grec & $P G$ & S1 & $\mathrm{Sm}$ & $\mathrm{S} 2$ \\
\hline \multirow[t]{2}{*}{$\delta$ бокв́ } & 27,8 & etpa. sidir & & istror \\
\hline & 40,37 & etpa. & etpa. & ivhor \\
\hline \multirow[t]{6}{*}{$\delta o ́ \xi \alpha$} & 27,8 & rusar & & Rhurard \\
\hline & 39,9 & rusar & rusar & 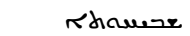 \\
\hline & 39,13 & rusar & ruscres & Rhwoend \\
\hline & 39,20 & Rhwared & Rhwourd & rusar \\
\hline & 40,10 & rusar & rusar & Rhwoned \\
\hline & 40,46 & Rurar & Rurar & Rhward \\
\hline$\delta o v \lambda \varepsilon v ́ \omega$ & 39,8 & אזגרבו. & אחגרמו. & ولu \\
\hline$\delta \rho \alpha ́ \sigma \sigma o \mu \alpha \imath$ & 40,39 & אחו & אחומ. & لحn \\
\hline$\delta \rho \alpha ́ \omega$ & 40,23 & حרו: & حרו: & iso \\
\hline \multirow[t]{13}{*}{$\delta v ́ v \alpha \mu \alpha \imath$} & 27,2 & תRבu & & S \\
\hline & 27,6 & مع ש & & דתى \\
\hline & 39,5 & & معصe & ه \\
\hline & 39,14 & תren & معحس & S \\
\hline & 40,4 & معصe & אשת & S \\
\hline & 40,11 & مع Rev & مع Rev & S \\
\hline & 40,18 & معen & معeve & S \\
\hline & 40,24 & معen & rer & S \\
\hline & 40,18 & ren & مع معu & S \\
\hline & 40,30 & reve & rex & S \\
\hline & 40,38 & reve & معصe & S \\
\hline & 40,39 & معحe & rer & S \\
\hline & 40,40 & תRe & مع & ד \\
\hline \multirow[t]{3}{*}{ 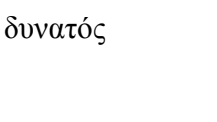 } & 27,5 & preve & p. معدس & p. \\
\hline & 38,10 & Rבשט p. & & p. \\
\hline & 40,25 & p. & p. معصع & p. \\
\hline$\delta v v \alpha \sigma \tau \varepsilon i ́ \alpha$ & 40,40 & Rhoustes & Rhouster & حمعنم \\
\hline$\delta v \sigma \alpha ́ \lambda \omega \tau \sigma \varsigma$ & 39,10 & Rhanea & רסחمולא הוברחזיא & trangar \\
\hline$\delta v \sigma \alpha v \alpha ́ \kappa \lambda \eta \tau ం \varsigma$ & 40,33 & 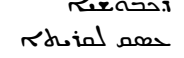 & حمه لحin & R Ridill \\
\hline$\delta v \sigma \kappa \alpha ́ \theta \varepsilon \kappa \tau о \varsigma$ & 27,5 & Rhoulatasl & & a.uutitasl \\
\hline$\delta v ́ \sigma \mu \alpha \chi o \varsigma$ & 39,10 & $\begin{array}{r}\text { dor readud } \\
\text { ح }\end{array}$ & مredadal Re & 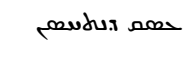 \\
\hline$\delta \omega \rho \varepsilon \alpha ́$ & $40,16^{47}$ & rhanel & rhanef & תחحשת \\
\hline \multirow[t]{2}{*}{$\delta \tilde{\omega} \rho \circ{ }^{\prime}$} & 40,4 & عaمur & عaحمr & Rh⿻mos \\
\hline & 40,4 & عمحلr & קمave & Rh⿻mos \\
\hline$\dot{\varepsilon} \gamma \varepsilon \dot{\rho} \rho \omega$ & 40,13 & סחסק & مחס & iedidir \\
\hline$\dot{\varepsilon} \gamma \kappa \sigma_{\sigma} \sigma \mu \mathrm{to}$ & 39,14 & ג--ב-حلsى & ג--ב-حلsى & حلsur \\
\hline$\dot{\varepsilon} \gamma \kappa \rho \alpha \tau \tilde{c}^{\prime}$ & 40,26 & रा & रा & Riأ \\
\hline$\dot{\varepsilon} \gamma \chi \alpha \rho \alpha ́ \sigma \sigma \omega$ & 40,26 & טiv & טivo & i \\
\hline$\dot{\varepsilon} \gamma \chi \varepsilon i \rho i ́ \zeta \omega$ & 27,6 & pa. غلم & & מחד טחמוזא \\
\hline$\dot{\varepsilon} \theta \varepsilon \dot{\varepsilon} \lambda \omega$ & 40,27 & (1) & טגה & रू \\
\hline عĩ $\delta \circ \varsigma$ & 39,16 & row & row & Rhis \\
\hline$\varepsilon i \delta \omega \lambda o v$ & 40,38 & Rhull & Rtull & Rhasi \\
\hline घi்ó $\tau \omega \varsigma$ & 40,20 & גoron & גoril & ב-abla \\
\hline 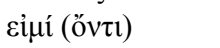 & 27,3 & River & & horizir \\
\hline 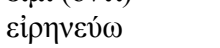 & 40,43 & etpa. אזغ etra & 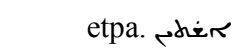 & مars rer \\
\hline 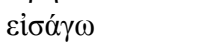 & 39,18 & حس & محلد af. & af. نشحل \\
\hline 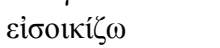 & 39,107 & כet, & af. & af. مخحكו \\
\hline$(-o \mu \alpha \imath)$ & 40,35 & حل لحentr & etpa. ,hoidra & حsi \\
\hline$\varepsilon \check{\kappa} \alpha \sigma \tau \mathrm{\alpha}$ & 40,34 & حلت & حلي & حلes. \\
\hline$\dot{\varepsilon} \kappa \delta \eta \mu i ́ \alpha$ & 40,11 & rwiar & rutiar & reow \\
\hline$\dot{\varepsilon} \kappa \kappa \alpha \rho \pi \varepsilon ́ \omega$ & 27,9 & rav & & ab \\
\hline 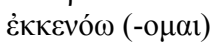 & 40,38 & ix Rhr & IrRdir & etpa. \\
\hline \multirow[t]{2}{*}{$\dot{\varepsilon} \kappa \lambda \dot{\alpha} \mu \pi \omega$} & 39,10 & af. & af. & אنגוזi \\
\hline & 40,6 & ins & ins & us \\
\hline$\tilde{\varepsilon} \kappa \lambda v \tau O \varsigma$ & 39,4 & & pa. p.p. غ غ & Ri \\
\hline 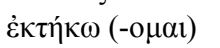 & 39,19 & etpa. مغنגم & etpa. אוגisa & אתגisi etpa \\
\hline
\end{tabular}

${ }^{47}$ Mais juste avant tous ont عمصنم pour rendre le même mot grec. 


\begin{tabular}{|c|c|c|c|c|}
\hline Grec & $P G$ & S1 & $\mathrm{Sm}$ & S2 \\
\hline$\dot{\varepsilon} \kappa \varphi \varepsilon ́ \rho \omega(-o \mu \alpha \imath)$ & 27,5 & Jir & & rivdir \\
\hline \multirow[t]{3}{*}{ हैкцороऽ } & 27,5 & rashos & & Rishoo \\
\hline & $(\mu \grave{\eta})$ & & & \\
\hline & 40,45 & rinastos & rinastos & rastoss \\
\hline$\dot{\varepsilon} \lambda \alpha \chi v ́ \varsigma$ & 40,19 & Rin & Rinl & حى .i م \\
\hline$\dot{\varepsilon} \lambda \varepsilon v \theta \varepsilon \rho i ́ \alpha$ & 40,13 & Rhairu & rhairse & Riöw \\
\hline \multirow[t]{4}{*}{$\ddot{\varepsilon} \lambda \lambda \alpha \mu \psi 1 \varsigma$} & 38,9 & Rhovimss & & Rhovimstas \\
\hline & $39,8^{1}$ & Rhaiss & rhovimstas & rhovimstos \\
\hline & $39,8^{2}$ & Rhaisms & Rhouimes & Rhouimstas \\
\hline & 40,36 & Rhaisss & Rhaiss & Rhouimstas \\
\hline$\varepsilon ้ \lambda \lambda \varepsilon 1 \psi 1 \varsigma$ & 39,11 & rives & ح5 & Rhais \\
\hline \multirow[t]{2}{*}{$\dot{\varepsilon} \lambda \pi i \zeta \omega$} & 40,11 & סصi & o & pa. فحر \\
\hline & 40,25 & סחוֹ & pa. فحر & pa. فحر \\
\hline$\dot{\varepsilon} \mu \pi \alpha \theta \dot{n} \varsigma$ & 40,2 & Nercos & Rencus & Nev \\
\hline$\dot{\varepsilon} \mu \pi \varepsilon \delta \delta^{\prime} \omega$ & 40,8 & pa. ن & pa. ن & pa. غi \\
\hline$\dot{\varepsilon} \mu \pi \varepsilon \rho \rho \varepsilon \kappa \tau \iota \kappa o ́ \varsigma$ & 40,3 & 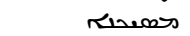 & 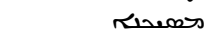 & Rrowo \\
\hline$\dot{\varepsilon} \mu \pi i ́ \pi \tau \omega$ & 40,7 & tow & tow & las \\
\hline$\dot{\varepsilon} \mu \varphi \omega ́ \tau \varepsilon 10 \varsigma$ & 40,25 & Rimas & Rists & Rhorosess-x \\
\hline$\dot{\varepsilon} v \alpha \gamma \kappa \alpha \lambda i \zeta{ }^{\prime} \mu \alpha \iota$ & 39,14 & ran & حaم & عمل حل הiּحم \\
\hline Ėvavtíos & 40,24 & סممحلي & סممحلה & ג-ل-ممحلح \\
\hline 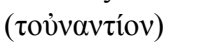 & 27,7 & 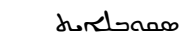 & & xلمحكلي \\
\hline 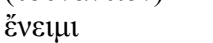 & 40,20 & J 1 ram & 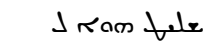 & J Ram dor \\
\hline$\dot{\varepsilon} v \varepsilon \rho \gamma \varepsilon \dot{\varepsilon} \omega$ & 39,1 & & iso & raf. \\
\hline$\varepsilon ้ v \theta \varepsilon \circ \varsigma$ & 39,14 & هلم ملهم & دلك ملهم & Risend \\
\hline Ěvvold & 38,12 & rhenith & & Rharus \\
\hline \multirow{2}{*}{$\begin{array}{l}\dot{\varepsilon} v \sigma \eta \mu \alpha i ́ v \omega \\
(-o \mu \alpha \imath)\end{array}$} & 40,32 & peritir & paridr & rosa $\downarrow$ rom \\
\hline & 40,36 & peridir & revidir & etpa. oaljir \\
\hline 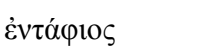 & 40,15 & محمin & محمiir & حمونr \\
\hline 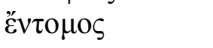 & 39,5 & & ris & pa. p.p. $\downarrow^{\circ}$ \\
\hline 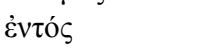 & 39,7 & x-1- & x- & ral \\
\hline$\tilde{\varepsilon} v \omega \sigma ı \varsigma$ & 40,43 & Rhonicul & rhionis & Rhon.w \\
\hline$\dot{\varepsilon} \xi \alpha \gamma o ́ \rho \varepsilon v \sigma 1 \zeta$ & 40,20 & R.sone & r.tane & rhwiod \\
\hline$\ddot{\varepsilon} \xi \alpha \lambda \mu \alpha$ & 40,5 & rosi & لحئ & rus \\
\hline \multicolumn{5}{|l|}{ 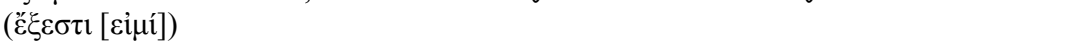 } \\
\hline$\dot{\varepsilon} \xi \varepsilon \tau \alpha \dot{\zeta} \omega(-о \mu \alpha)$ & 27,3 & خمد pa.p.p. & & p.p. \\
\hline$\tilde{\varepsilon} \xi \xi \varsigma$ & 40,36 & 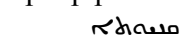 & مeسمh & welv \\
\hline$\dot{\varepsilon} \xi \hat{\jmath} \sigma \tau \eta \mu \iota$ & 39,12 & af. & af. & קن. pa. \\
\hline$\dot{\varepsilon} \xi o ́ \delta 10 \zeta$ & 40,12 & roal & תمas & sمar \\
\hline 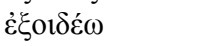 & 27,7 & 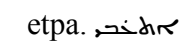 & & uas \\
\hline$\dot{\varepsilon} \pi \alpha \imath v \varepsilon \tau o ́ \varsigma$ & 27,2 & Sתבטית & & sملمجr \\
\hline 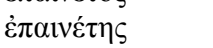 & 40,32 & קحس p.p.p. & غحس . pa. p.p. & فله pa.p.p. \\
\hline \multirow[t]{2}{*}{ 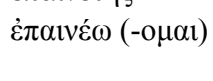 } & 39,9 & etpa. & 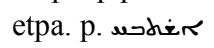 & riodots \\
\hline & 27,7 & $\begin{array}{r}\text { p. } \\
\text { Rhasi }\end{array}$ & & Rhaness \\
\hline$\varepsilon \dot{\pi} \varepsilon^{\prime} \gamma \omega(-\mathrm{o} \mu \alpha \imath)$ & 40,24 & Imikor & Imikor & etpa. taidir \\
\hline 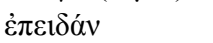 & 39,2 & & אתדנ, י. & Sטגוא ה \\
\hline$\dot{\varepsilon} \pi \varepsilon ́ \rho \chi 0 \mu \alpha \imath$ & 40,10 & rhir & rdir & r \\
\hline$\dot{\varepsilon} \pi \varepsilon \dot{\chi} \chi \omega(-o \mu \alpha \imath)$ & 40,11 & nathor & undir & dadir \\
\hline$\dot{\varepsilon} \pi \eta \rho \varepsilon \alpha ́ \zeta \omega$ & 27,6 & صا حملأم & & ロ \\
\hline$\dot{\varepsilon} \pi \eta ́ \rho \varepsilon 1 \alpha$ & 38,12 & من & & 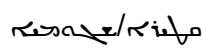 \\
\hline$\dot{\varepsilon} \pi \imath \beta \alpha i ́ v \omega$ & 40,10 & nix & nix & 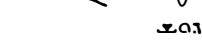 \\
\hline$\dot{\varepsilon} \pi \imath \beta 0 v \lambda \varepsilon v ́ \omega$ & 40,16 & pa. & pa. & af. منحص \\
\hline$\dot{\varepsilon} \pi 1 \beta 00 \lambda \eta ́$ & 40,16 & تحنك & טריא & Rhanes \\
\hline 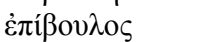 & 40,12 & rayers & Ray & דריאה \\
\hline$\dot{\varepsilon} \pi \dot{\gamma} \gamma \nu \omega \sigma t \varsigma$ & 39,10 & 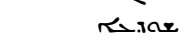 & rhanedos & rhouriaturs \\
\hline 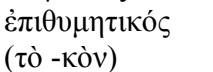 & 40,40 & rati & rati & rhash ai \\
\hline$\dot{\varepsilon} \pi 1 \kappa \alpha ́ \lambda v \psi 1 \varsigma$ & 40,32 & rheord & rheord & Rhuaud \\
\hline \multirow{2}{*}{$\dot{\varepsilon} \pi \mu \varepsilon \lambda \dot{\eta} \varsigma(-\tilde{\omega} \varsigma)$} & 27,5 & 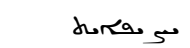 & & حمل \\
\hline & 39,14 & h० & 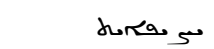 & حمل \\
\hline
\end{tabular}




\begin{tabular}{|c|c|c|c|c|}
\hline Grec & $P G$ & $\mathrm{~S} 1$ & $\mathrm{Sm}$ & S2 \\
\hline \multirow[t]{3}{*}{$\dot{\varepsilon} \pi \dot{\pi} \pi$ ovo } & 39,17 & af.p. & حيكلم & حدملح \\
\hline & 39,19 & 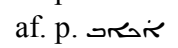 & حدمله & حهلم \\
\hline & 40,9 & p. & pمد & حدمله \\
\hline$\dot{\varepsilon} \pi \iota \sigma \kappa \varepsilon \dot{\varepsilon} \pi \tau \rho \mu \alpha \iota$ & 40,20 & etpa. vidir & etpa. sidir & عלخ etpa. \\
\hline$\dot{\varepsilon} \pi 1 \sigma \tau \rho \circ \varphi \eta ́$ & 39,17 & Rhoungestas & rdiongentos & rhowedos \\
\hline$\dot{\varepsilon} \pi 1 \sigma \varphi \alpha \lambda \eta \dot{~}$ & 40,18 & 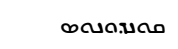 & 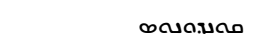 & Rim \\
\hline$\dot{\varepsilon} \pi \imath \tau^{\prime} \theta \eta \mu$ & 40,10 & סחק & סחקד & dحב \\
\hline$\dot{\varepsilon} \pi \imath \tau \gamma \gamma \chi \dot{\alpha} v \omega$ & 27,10 & $=4$ & & nis \\
\hline$\dot{\varepsilon} \pi ı \varphi \alpha ́ v \varepsilon ı \alpha$ & 40,24 & रoL & row & xw. \\
\hline 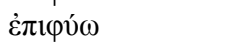 & 27,4 & סחס & & ערב \\
\hline$\dot{\varepsilon} \pi \omega ́ v 0 \mu \mathrm{o} \varsigma$ & 40,3 & حis & טi عss & عaم כתה \\
\hline \multirow[t]{5}{*}{$\dot{\varepsilon} \rho \gamma \alpha ́ \zeta$ o $\mu \alpha 1$} & 39,10 & ram & ram & حרו: \\
\hline & 40,9 & i2o & $i \infty$ & حבו. \\
\hline & 40,13 & iు & & ولe \\
\hline & 40,13 & ivo & in & ولe \\
\hline & 40,45 & $+\infty$ & is & ولe \\
\hline 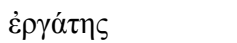 & 40,24 & p. & p. حيل & p. \\
\hline$\varepsilon \rho \varepsilon \sigma \chi \eta \lambda i ́ \alpha$ & 27,3 & iهم & & Rhonitu \\
\hline है $\rho \omega \varsigma$ & 27,10 & Rhoravi & & Rhosui \\
\hline \multirow[t]{2}{*}{ 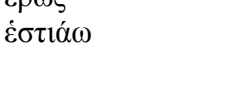 } & 27,2 & soith & & pa. ضمه \\
\hline & 40,46 & حבו: Rhair & حרו: Rhair & pa. خمه \\
\hline$\varepsilon \tilde{\tilde{~}} \gamma^{\prime} \gamma \nu 0 \mu \alpha \mathrm{l}$ & 40,26 & rom inar & pa. غr & rom ivar \\
\hline$\varepsilon \tilde{\tilde{U}} \pi \mathrm{ol \varepsilon ́} \omega$ & 40,13 & حבו: לרחמג & حרז לגתמג & 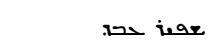 \\
\hline \multirow[t]{2}{*}{ 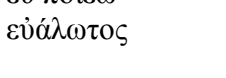 } & 39,18 & hablex & Rhouratos dialedx & tronerg \\
\hline & & Rhouratas & & 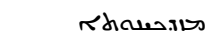 \\
\hline 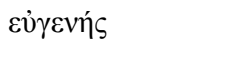 & 27,1 & scrat & & riru \\
\hline 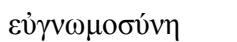 & 40,43 & عمحملم & rhat Rhuid & rhelid datiag \\
\hline$\varepsilon \cup ่ \gamma \nu \omega ́ \mu \omega \nu$ & 27,5 & rhenid itar & & \\
\hline 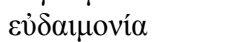 & 39,5 & & rhas dialf & حחקח \\
\hline$\varepsilon v ̉ \delta i \alpha ́ \lambda v \tau o \varsigma$ & 39,15 & Rivl Rarg & rivl Rave & riv duld \\
\hline \multirow{2}{*}{ 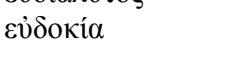 } & 38,15 & rhoules 5 & & Rhauld \\
\hline & 38,15 & & & rhant s \\
\hline \multirow[t]{6}{*}{$\varepsilon v ̉ \varepsilon \rho \gamma \varepsilon \sigma i ́ \alpha$} & 40,1 & حمוiה ה & حمיוֹט & Rhand haineo \\
\hline & 40,4 & حمוiיטת & حחוiה ה & rhant diaine \\
\hline & 40,11 & حمוiיה & حחוiה ה & rhant diaineo \\
\hline & 40,27 & rition & rition & حمונוז r \\
\hline & 40,31 & حمוis & حחוist & doivar \\
\hline & & & & Rhaines \\
\hline$\varepsilon \cup ̉ \varepsilon \rho \gamma \varepsilon \tau \varepsilon ́ \omega$ & 39,10 & & عariti حרו. & خ خ \\
\hline$(-o \mu \alpha 1)$ & 40,27 & ids & ids & etpa. indidr \\
\hline \multirow[t]{2}{*}{ 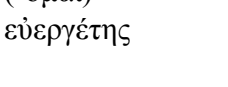 } & 38,11 & ritiass & & 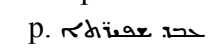 \\
\hline & 40,12 & sins & S rins & p. مमتلخ \\
\hline$\varepsilon v ̉ \eta ́ \theta \varepsilon ı$ & 40,27 & Rhative & Rhowe/rhater & Jan diateas \\
\hline$\varepsilon v ่ \theta v ́ \varsigma$ & 40,39 & rah & reth & $r_{5}$ ih \\
\hline 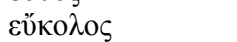 & 27,4 & x.ele & & Ro.ro \\
\hline 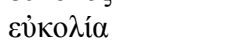 & 40,27 & rhales. & rhales & Rhonera \\
\hline \multirow[t]{2}{*}{$\varepsilon v \dot{\lambda} \lambda \dot{\alpha} \beta \varepsilon 1 \alpha$} & 39,14 & rondur & rotur. & من \\
\hline & 40,16 & Rorlur & rhan & 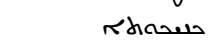 \\
\hline \multirow[t]{2}{*}{$\varepsilon \cup ̉ \mu \varepsilon \tau \alpha \kappa i ́ v \eta \tau o \varsigma$} & 40,44 & hundelx & xلdedidos & $\int_{e} x$ \\
\hline & & & & rhamididost \\
\hline \multirow[t]{3}{*}{ 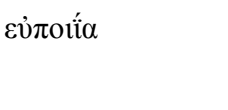 } & 40,13 & rholl & Rhaioseo daivar & doivar \\
\hline & & & & Rhainso \\
\hline & 40,33 & Rhon & Rhoil & حחוis \\
\hline$\varepsilon \cup ̉ \pi o \rho \varepsilon ́ \omega$ & 40,18 & pa. ids & wdir & sutir \\
\hline 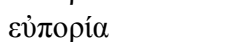 & 40,34 & Rhaidus & Rhoutives & Rhoutives \\
\hline \multirow[t]{2}{*}{$\varepsilon v ̉ \sigma \varepsilon ́ \beta \varepsilon 1 \alpha$} & 27,2 & הטلג ملهم & & Rolus diainar \\
\hline & 40,16 & הטلג ملهم & תטגוh תאלחת & Rolus diainar \\
\hline & 39,5 & & & عavi تحله \\
\hline \multirow[t]{2}{*}{$\varepsilon v ̋ \sigma \tau \rho \circ \varphi{ }^{\prime}$} & 27,1 & xلs & & ملeله \\
\hline & & כתחפרת & & \\
\hline$\varepsilon v ่ \sigma \chi \eta ́ n \omega \nu$ & 40,37 & inar & 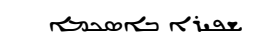 & 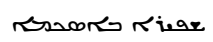 \\
\hline$(-\mu o ́ v \omega \varsigma)$ & & durblosver & & \\
\hline 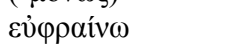 & 40,38 & रisu & R.w & pa. خمم \\
\hline 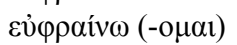 & 39,20 & אur & טwi & 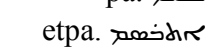 \\
\hline
\end{tabular}




\begin{tabular}{|c|c|c|c|c|}
\hline Grec & $P G$ & $\mathrm{~S} 1$ & $\mathrm{Sm}$ & $\mathrm{S} 2$ \\
\hline 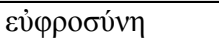 & 40,38 & Rhares & Kha.w & ح'son \\
\hline 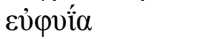 & 38,10 & 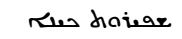 & & Khowh \\
\hline$\varepsilon v ่ \omega \delta i ́ \alpha$ & 27,3 & Rhosean & & Rusididasean \\
\hline 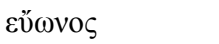 & 27,3 & $r a r$ & & عهم כוכה \\
\hline \multirow[t]{2}{*}{$\dot{\varepsilon} \varphi \dot{\eta} \mu \imath$} & 39,8 & $\lambda i$ & $\lambda_{i}$ & etpa. \\
\hline & 39,18 & af. & af. صن & عרم \\
\hline 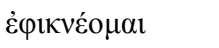 & 27,3 & As & & af. אזניטר \\
\hline 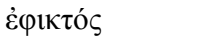 & 39,7 & p. quistir & p. & p. \\
\hline \multirow[t]{2}{*}{$\zeta \eta \mu i ́ \alpha$} & 40,24 & rioon & rions & סח >ריم \\
\hline & 40,24 & کiloow & Nion & סم حar \\
\hline$\zeta \omega о \pi$ oté $\omega$ & 40,43 & حרו تשـ & af. & af. \\
\hline \multirow[t]{2}{*}{$\zeta \omega \tau \imath \kappa o ́ \varsigma$} & 39,20 & ver & rev & תחשr \\
\hline & 40,10 & مev & Nev & תטerr \\
\hline \multirow[t]{4}{*}{ 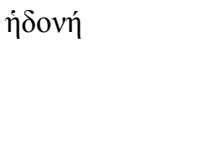 } & 40,14 & rमners & rhaves & rari \\
\hline & 40,17 & rhaver & rhaves & rari \\
\hline & 40,20 & rhowm & rhowm & rh⿻二丨 \\
\hline & 40,33 & rdr & rhaves & rdi \\
\hline$\eta ँ \sigma \sigma \omega \nu$ & 39,20 & גorinin & 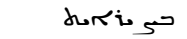 & حك \\
\hline$\tilde{\eta} \chi \circ \varsigma$ & 39,4 & & 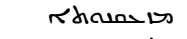 & గحai \\
\hline 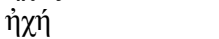 & 39,4 & & مله & ${ }{ }_{5}$ \\
\hline$\theta \alpha \lambda \alpha ́ \sigma \sigma ı \varsigma$ & 39,14 & 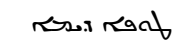 & טמתה & טמקה \\
\hline$\theta \alpha \dot{\lambda} \lambda \omega$ & 40,33 & $10 i$ & $10 i$ & p.p. Jd \\
\hline$\theta \alpha \tilde{v} \mu \alpha$ & 40,29 & Rhr & rdir & Rhinsid \\
\hline$\theta \alpha v \mu \alpha \sigma \tau o ́ s$ & 40,30 & Rhicsid & Rhicsid & rosesh \\
\hline 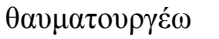 & 40,18 & Rhत̈rsh iح & 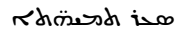 & Rhiosidi ivo \\
\hline \multirow[t]{2}{*}{$\theta \varepsilon \dot{\alpha}$} & 40,8 & row & row & Rhiw \\
\hline & 40,38 & row & row & Khiw \\
\hline \multirow[t]{4}{*}{$\theta \varepsilon \circ \lambda \circ \gamma i ́ \alpha$} & 27,3 & rarks & & diallas \\
\hline & & & & Rhand \\
\hline & $27,6^{48}$ & rand & & siallas \\
\hline & & & & Rhand \\
\hline \multirow[t]{2}{*}{$\theta \varepsilon \circ \lambda$ ó } & 27,7 & on alard & & 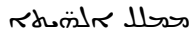 \\
\hline & 27,9 & तarah & & 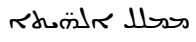 \\
\hline \multirow[t]{2}{*}{$\theta \varepsilon \circ \varphi \alpha ́ v 1 \alpha$} & 27,9 & 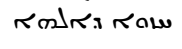 & & darists \\
\hline & & & & rod \\
\hline$\theta \varepsilon o ́ \omega(-o \mu \alpha \imath)$ & 40,42 & rm & 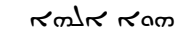 & alikdir \\
\hline$\theta \varepsilon \rho \alpha \pi \varepsilon i ́ \alpha$ & 39,9 & טمل & rastav & rhacor \\
\hline \multirow[t]{2}{*}{ 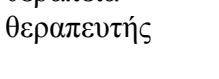 } & 39,9 & תسلsلr & תملsir & 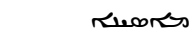 \\
\hline & 40,12 & תחת & תم: & reor \\
\hline$\theta \varepsilon \omega \rho \eta \tau \tilde{\omega} \varsigma$ & 40,8 & riand-ב & riard-ב & doriluds \\
\hline \multirow[t]{2}{*}{$\theta \eta \lambda v ́ v \omega$} & 27,9 & pa. & & i \\
\hline & 40,38 & 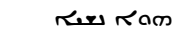 & ram ram & etpa. \\
\hline$\theta \eta \rho i ́ o v$ & 40,45 & Khanes & Rhones & Ser dices \\
\hline$\theta \eta \sigma \alpha v \rho i ́ \zeta \omega$ & 40,33 & خע pa. & خע pa. & vas \\
\hline$\theta \rho \imath \alpha \mu \beta \varepsilon v ́ \omega$ & 40,27 & pa. i أنس & i حس /pa. حسi & aig, \\
\hline$\theta v \rho \varepsilon o ́ \varsigma$ & 40,16 & هָא & דָה & סحiir \\
\hline ¡ $\delta$ เó $\tau \rho \circ \pi \circ \varsigma(-\tilde{\omega} \zeta)$ & 40,6 & صاكم ז.ملسك & حالم ז.ملبك & 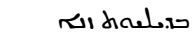 \\
\hline ĩ $\pi \pi \circ \varsigma$ & 27,5 & గomo & & Tri \\
\hline 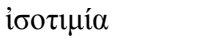 & 40,18 & 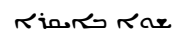 & Rinow dian & Rinor dions \\
\hline i $\sigma \tau о \rho \varepsilon ́ \omega(-о \mu \alpha \imath)$ & 40,30 & etpa. sdidir & etpa. stidir & pridir \\
\hline \multirow[t]{3}{*}{ 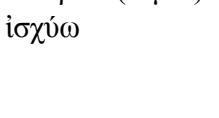 } & 40,16 & RתR & Rer & 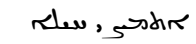 \\
\hline & 40,16 & معحس & معרu & 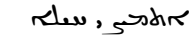 \\
\hline & 40,33 & Rבת & Rer & $r_{5}$ \\
\hline$\kappa \alpha \theta \alpha ́ \alpha \varepsilon \rho$ & 27,5 & s & & حس אח \\
\hline \multirow[t]{5}{*}{$\kappa \alpha ́ \theta \alpha \rho \sigma ı$} & 39,3 & & rherdi & תم \\
\hline & 39,8 & Rherid & rherdh & תחסح \\
\hline & 39,15 & אירגה & hodrar & תחסح \\
\hline & 40,7 & זירסאי & 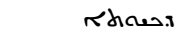 & נת. \\
\hline & 40,18 & תחם & תחת & 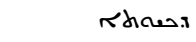 \\
\hline
\end{tabular}

${ }^{48}$ Mais plus haut en 27,4, le mot est rendu par ra a dark en S1 et S2, et

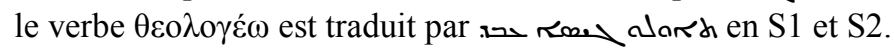




\begin{tabular}{|c|c|c|c|c|}
\hline Grec & $P G$ & $\mathrm{~S} 1$ & $\mathrm{Sm}$ & $\mathrm{S} 2$ \\
\hline & 40,29 & 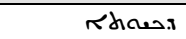 & Rhovis & תח \\
\hline \multirow[t]{2}{*}{$\kappa \alpha \theta \varepsilon v ́ \delta \omega$} & $40,13^{49}$ & عרב & عحב & זית \\
\hline & 40,13 & זיקו & xדי & عحב \\
\hline$\kappa \alpha \iota v о \tau о \mu \varepsilon ́ \omega$ & 39,13 & soadir & Rhatiuu حadir & مidi etpa. \\
\hline$(-o \mu \alpha \imath)$ & & Rhaditue & & \\
\hline каí $\varepsilon \rho \rho$ & 27,5 & حدم م/ & & חحי לل \\
\hline ка1рós & 40,10 & احلم & احلم & ح. كم \\
\hline 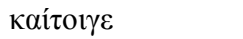 & 27,8 & గma & & כי. حم \\
\hline$\kappa \alpha i ́ \omega$ & 40,6 & af. החمסה & af. & Jial \\
\hline \multirow[t]{2}{*}{$\kappa \alpha \kappa о \delta \alpha i ́ \mu \omega \nu$} & 39,3 & & حי & 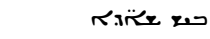 \\
\hline & 39,3 & & حי & حש ع عتّאم \\
\hline$\kappa \alpha \kappa o \delta \circ \xi i \alpha$ & 38,16 & 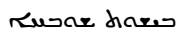 & & Rhousen hare \\
\hline 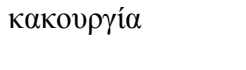 & 40,20 & $\begin{array}{l}\text { doinso } \\
\text { Rhore. }\end{array}$ & rhare diaino & Rhasivs \\
\hline $\begin{array}{l}\kappa \alpha \kappa о \tilde{\nu} \rho \gamma о \varsigma \\
(-\omega \varsigma)\end{array}$ & 40,21 & $\begin{array}{l}\text { dainver } \\
\text { Rhores }\end{array}$ & כuinaris & donsitss \\
\hline \multirow[t]{2}{*}{$\kappa \alpha \lambda o ́ \varsigma$} & 27,4 & له & & Riear \\
\hline & 40,18 & Riear & rivar & तु \\
\hline \multirow[t]{3}{*}{$(-\tilde{\omega} \zeta)$} & 27,4 & גont & & dorkiear \\
\hline & 39,9 & hond & hon & horiegr \\
\hline & 40,32 & גم & 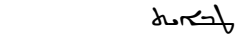 & iear \\
\hline \multirow[t]{2}{*}{$\kappa \alpha ́ \lambda v \mu \mu \alpha$} & 39,9 & Kheord & rheand & Kheand \\
\hline & 40,4 & דרم.0م & & r.ans \\
\hline$\kappa \alpha \pi \eta \lambda \imath \kappa o ́ \varsigma$ & 40,20 & مو.لح & مو.له & ation $R$ r \\
\hline$\kappa \alpha \rho \pi о \varphi о \rho \varepsilon ́ \omega$ & 39,9 & $\begin{array}{r}\text { af. } \\
\text { af. }\end{array}$ & 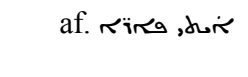 & فiأح صعiّم pa. \\
\hline$\kappa \alpha \rho \pi o ́ \omega$ & 40,24 & గal & ra & ملa \\
\hline$\kappa \alpha \tau \alpha \beta \alpha ́ \lambda \lambda \omega$ & 27,7 & af. منهل & & si \\
\hline$\kappa \alpha \tau \alpha ́ \gamma \nu \omega \sigma ı \varsigma$ & 39,18 & حה.לה & حה.لה & rhadidist \\
\hline$\kappa \alpha \tau \alpha ́ \gamma \chi \omega$ & 39,18 & טلم & exh & a d \\
\hline$\kappa \alpha \tau \alpha \delta \rho о \mu \eta ́$ & 39,10 & तهi & सهi & 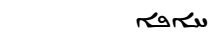 \\
\hline 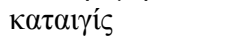 & 40,36 & KhLL Ruai & RhLL Ruai & حلى \\
\hline $\begin{array}{l}\kappa \alpha \tau \alpha \lambda \alpha \mu \beta \alpha ́ v \omega \\
(-o \mu \alpha \iota)\end{array}$ & 39,2 & & nixdir & مאiفi etpa. \\
\hline$\kappa \alpha \tau \alpha ́ \lambda v \sigma ı \varsigma$ & 40,3 & 反ix & كir & ritho \\
\hline$\kappa \alpha \tau \alpha \mu \alpha v \theta \alpha \dot{v} \omega \omega$ & 40,20 & $a / r$ & $a /$ r & عiخمر etpa \\
\hline $\begin{array}{l}\kappa \alpha \tau \alpha \pi t \varepsilon ́ \zeta \zeta \omega \\
(-о \mu \alpha \imath)\end{array}$ & 27,3 & $\begin{array}{r}\text { etpa. } \\
\text { etpa }\end{array}$ & & $s$ stir \\
\hline$\kappa \alpha \tau \alpha ́ \alpha \chi \varepsilon \sigma 1 \varsigma$ & 40,35 & & rivs & حries du \\
\hline $\begin{array}{l}\kappa \alpha \tau \alpha \tau \rho v \varphi \alpha ́ \omega \\
(-о \mu \alpha \imath)\end{array}$ & 39,5 & & etpa. ملح م & etpa. \\
\hline$\kappa \alpha \tau \alpha \varphi \varepsilon ́ \rho \omega$ & 40,10 & dow & dow & Ja \\
\hline$\kappa \alpha \tau \alpha \varphi \varepsilon v ́ \gamma \omega$ & 39,7 & حilo & af.hwre & etpa. on \\
\hline$\kappa \alpha \tau \alpha \psi v ́ \chi \omega(-о \mu \alpha \imath)$ & 40,11 & مiٔi & קרישr & مרerer \\
\hline \multirow[t]{2}{*}{$\kappa \alpha \tau \varepsilon ́ \chi \omega$} & 40,38 & .wo & two & لحn \\
\hline & 40,32 & لרu & لרu & ת \\
\hline 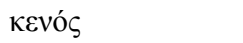 & 40,46 & مiأمك & مiأمك & م \\
\hline 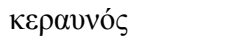 & 39,4 & & حiiمك & همحم \\
\hline \multirow[t]{3}{*}{$\kappa \varepsilon \varphi \alpha ́ \lambda \alpha 10 \varsigma$} & 38,16 & ri & & مaلمه - \\
\hline & 39,11 & i i & مaلمهم & مaلمج \\
\hline & 40,18 & Rri & مaلمa م- & durases \\
\hline$\kappa \eta \rho v ́ \sigma \sigma \omega \mathrm{p}$. & 40,26 & תحiiاسم & תרוֹות & रावi \\
\hline \multirow[t]{2}{*}{ 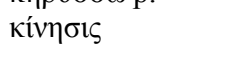 } & 39,5 & & مح & Rhaniditos \\
\hline & 39,8 & 四 & 四 & rhanididos \\
\hline 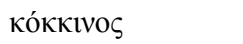 & 39,20 & مחתדم & مחדתمr & riavl \\
\hline$\kappa о \lambda \alpha \kappa \varepsilon i ́ \alpha$ & 27,9 & rhary & & Rhoudear $x$ \\
\hline 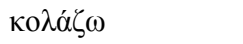 & 38,12 & غنم pa. & & $\alpha i$ \\
\hline \multirow[t]{3}{*}{$\kappa o ́ \lambda \alpha \sigma ı \varsigma$} & 39,5 & & عمدمك & كعمة حiأعك \\
\hline & 40,9 & תمم حiأعم & عمدمك & תممر حأنعم \\
\hline & 40,27 & عملمك & عمدمك & rowed \\
\hline
\end{tabular}

${ }^{49}$ Dans une citation biblique (Eph 5,14). 


\begin{tabular}{|c|c|c|c|c|}
\hline Grec & $P G$ & $\mathrm{~S} 1$ & $\mathrm{Sm}$ & $\mathrm{S} 2$ \\
\hline 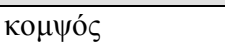 & 27,1 & תרוֹ & & p. Jinitir \\
\hline 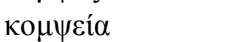 & 27,3 & محi & & مَini \\
\hline ко́ $\pi \mathrm{o} \varsigma$ & 40,24 & Rhand & Rhan & حدله \\
\hline ко́ $\pi \rho \circ$ & 39,15 & إم-له & امحله & احلكه \\
\hline 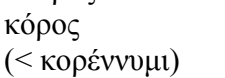 & 27,4 & סحרז & & Rhonos \\
\hline 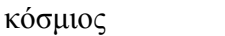 & 27,5 & rhang & & טשרחה R \\
\hline 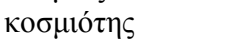 & 27,5 & Rhoras & & בשרתמא \\
\hline 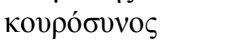 & 40,1 & 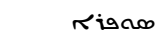 & Risao & 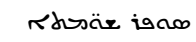 \\
\hline \multirow[t]{3}{*}{ кой } & 27,9 & 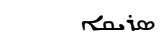 & & rueve \\
\hline & 39,15 & ris & ملبله & ملفيح \\
\hline & 40,17 & pa. له ف & Rueng & rueng \\
\hline 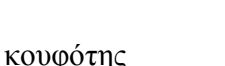 & 39,19 & $\begin{array}{r}\text { p.p. } \\
\text { रमمח.900 }\end{array}$ & Rhan.900 & م rialed \\
\hline$\kappa \rho \alpha ́ \sigma \pi \varepsilon \delta \circ \nu$ & 40,33 & Aabis & Afatis & र्य \\
\hline$\kappa \rho \alpha \tau \varepsilon ́ \omega$ & 40,16 & אחד & אח & آص \\
\hline \multirow[t]{6}{*}{ 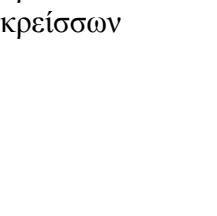 } & 38,15 & ridus & & همب \\
\hline & 40,19 & iֹ & וֹדת & مَ \\
\hline & 40,26 & iוֹת & iוֹת & ritus \\
\hline & 40,28 & همur & ومur & ritues \\
\hline & 40,36 & n & ritus & ridus \\
\hline & 40,45 & रो & ritus & ridus \\
\hline$\kappa \rho \eta \mu v i ́ \zeta \omega$ & 40,14 & af. גovi & af. מה מה & R.x \\
\hline 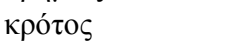 & 39,4 & & لقعم & لمع ونم \\
\hline$\kappa v \kappa \lambda 1 \kappa o ́ \varsigma$ & 40,5 & Ruinow & טחוitr & 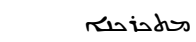 \\
\hline$\kappa \omega ́ \lambda v \mu \alpha$ & 40,27 & حمدivr & حمدír & حمחתم \\
\hline \multirow[t]{8}{*}{$\lambda \alpha \mu \beta \alpha ́ v \omega$} & 38,11 & lar & & נos \\
\hline & 39,1 & & عمل & נח \\
\hline & 39,1 & & عمل & Sos \\
\hline & 39,12 & נمב & عمل & נo \\
\hline & 40,5 & عمل & عمل & נחבר \\
\hline & 40,21 & عمل & נסב & נסב \\
\hline & 40,26 & נחב & נחב & אתו. \\
\hline & 40,37 & נסבר & נonב & את \\
\hline$\lambda \alpha \mu \pi \eta \delta \omega ́ v$ & 39,9 & r.uasl & rivast & russ \\
\hline$\lambda \dot{\alpha} \mu \psi 1 \varsigma$ & 40,37 & Rhoimes & Rhovimss & 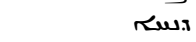 \\
\hline$\lambda \alpha \nu \theta \alpha ́ v \omega(-o \mu \alpha \imath)$ & 27,5 & 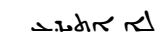 & & doredy \\
\hline 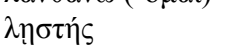 & 40,24 & ארבא & حلרת & rifol \\
\hline$\lambda \dot{x}{ }^{\prime} \operatorname{sos}$ & 38,12 & 出弐 & & لרבא \\
\hline $\begin{array}{l}\lambda \circ \beta \text { ós } \\
(\lambda . \eta ँ \pi \alpha \tau \sigma \varsigma)\end{array}$ & 40,40 & & טs iأ حصiم & \\
\hline 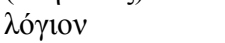 & 27,9 & shls & & 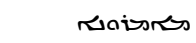 \\
\hline \multirow[t]{2}{*}{$\lambda$ oıㄷós } & 27,4 & i & & Rhares \\
\hline & 40,16 & rhenih & Rhenit & Rharus \\
\hline 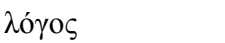 & 39,2 & & ritsos & مuls \\
\hline$\lambda v \sigma ı \tau \varepsilon \lambda \eta ́ s$ & 38,12 & حוk & & ruitas \\
\hline$\mu \alpha \theta \eta \tau \varepsilon i ́ \alpha$ & 40,44 & raba & rabas & rivslad \\
\hline$\mu \alpha \theta \eta \tau \varepsilon v ́ \omega$ & 40,45 & af.ala & af.ala & iscosh \\
\hline 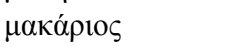 & 27,8 & raf & & rstart \\
\hline$\mu \alpha \lambda \alpha \kappa o ́ \varsigma(-\tilde{\omega} \varsigma)$ & 40,38 & i do do & 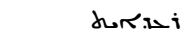 & horai \\
\hline$\mu \alpha{ }^{\prime} \lambda \alpha(-1 \sigma \tau \alpha)$ & 39,10 & rharelo & drriod & rachan \\
\hline$\mu \varepsilon \gamma \alpha \lambda \operatorname{có}^{\prime} \omega \rho \circ \varsigma$ & 40,27 & i זכ عمحلم & וֹד عمحلم & iוֹ \\
\hline \multirow[t]{2}{*}{$\mu \varepsilon \gamma \alpha \lambda \mathrm{o} \pi \rho \varepsilon \pi \eta \eta_{\varsigma}$} & 40,3 & iٓحس & 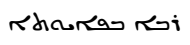 & 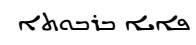 \\
\hline & & रhorरas & & \\
\hline \multirow[t]{2}{*}{ 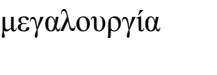 } & 38,11 & hasi & & 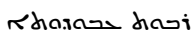 \\
\hline & & Rhanais & & \\
\hline \multirow[t]{2}{*}{$\mu \varepsilon \theta \dot{\varepsilon} \lambda \kappa \omega$} & 39,7 & W & W & af. مُحصi \\
\hline & 40,11 & pa.,mdi & pa.,msi & pa.na \\
\hline$\mu \varepsilon ı o ́ \omega(-o \mu \alpha \imath)$ & 40,41 & is & I & etpa.i_idis \\
\hline$\mu \varepsilon \lambda \alpha ́ v \omega \sigma i \varsigma$ & 40,19 & Rhonaru & Rhossar & rhassas \\
\hline$\mu \varepsilon \dot{\varepsilon} \lambda \lambda \eta \sigma i \varsigma$ & 40,25 & remat & rmat & rharess \\
\hline \multirow[t]{2}{*}{$\mu \varepsilon \dot{v} v \omega$} & 39,12 & فم,رpa & فم,.pa & pa.id \\
\hline & 40,24 & ins & ins & فa.,pa \\
\hline (p.) & 40,38 & rithes & Sithes & فم,pa.pa \\
\hline 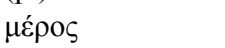 & 40,34 & Rhus & rous & אחוז \\
\hline
\end{tabular}




\begin{tabular}{|c|c|c|c|c|}
\hline Grec & $P G$ & S1 & $\mathrm{Sm}$ & $\mathrm{S} 2$ \\
\hline$\mu \varepsilon \dot{\varepsilon} \sigma \circ \varsigma$ & 40,6 & כהנ, & כeנ, & rh \\
\hline \multirow[t]{2}{*}{$\mu \varepsilon \tau \alpha \delta i ́ \delta \omega \mu$} & 27,5 & af.tran & & מחת \\
\hline & 39,18 & فلح & فلــ pa & st \\
\hline 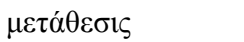 & 40,3 & 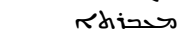 & shing & rwar \\
\hline \multirow{2}{*}{ 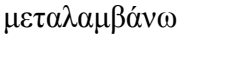 } & 40,31 & عمل هم & عمل هم & נمد דק \\
\hline & 40,46 & נחב & נחב & abiater \\
\hline$(-о \mu \alpha \imath)$ & 38,12 & rostir & & Jak \\
\hline$\mu \varepsilon \tau \alpha \lambda \eta \pi \tau \sigma^{\prime} \varsigma$ & 38,12 & וגם גנסב. & & 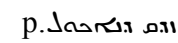 \\
\hline \multirow[t]{3}{*}{$\mu \varepsilon \tau \alpha \pi i \pi \tau \omega$} & 39,12 & etpa.Jaidir & حרi: & las \\
\hline & 39,12 & etpa.Jaidre & حصi دول & aluder \\
\hline & 39,12 & etpa.Jaidro & la & aludere \\
\hline$\mu \varepsilon \tau \alpha \pi \mathrm{o} \varepsilon \dot{\varepsilon} \omega(-\mathrm{o} \mu \alpha \mathrm{l})$ & 40,42 & ngendir & aluder & aludere \\
\hline$\mu \varepsilon \tau \alpha ́ \rho \sigma 10 \varsigma$ & 27,9 & si & & p.irर \\
\hline $\begin{array}{l}\mu \varepsilon \tau \varepsilon \gamma \gamma \rho \alpha \dot{\alpha} \varphi \omega \\
(-о \mu \alpha \imath)\end{array}$ & 40,44 & حרi مיdadis & stadir aluder & shadir ungm \\
\hline$\mu \varepsilon \tau \rho \varepsilon \dot{\varepsilon} \omega$ & 39,5 & & af. הنحسل & sers \\
\hline 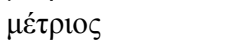 & 27,3 & ring & & pa.p.p.urs \\
\hline$(-\omega \varsigma)$ & 39,20 & 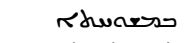 & doreurss & doreursss \\
\hline$\mu \eta \delta \alpha \mu \tilde{\omega} \varsigma$ & 27,5 & هم حل حله & & 徆 \\
\hline$\mu \eta \kappa \varepsilon ́ \tau 1$ & 40,33 & ه ת תמلl & دم תחعل & rad \\
\hline$\mu \dot{1} \mu \eta \sigma 1 \varsigma$ & 39,7 & rhasis & 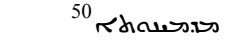 & resax \\
\hline$\mu \imath \mu v \eta ́ \sigma \kappa \omega(-o \mu \alpha \imath)$ & 27,7 & ividir & & حחות \\
\hline$\mu 1 \kappa \rho о \lambda о \gamma i \alpha$ & 40,34 & Rats doind & 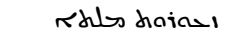 & Rhoraw \\
\hline \multirow{2}{*}{$\mu 1 \kappa \rho о \lambda о ́ \gamma о \varsigma$} & 27,10 & 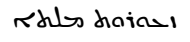 & & תרז \\
\hline & 39,16 & ורחi sلrals & 1 & וحمi נar \\
\hline$\mu 1 \kappa \rho o ́ \varsigma$ & 40,19 & ملeله & ملed & Rin \\
\hline \multirow[t]{2}{*}{$\mu \imath \sigma \alpha \nu \theta \rho \omega \pi i ́ \alpha$} & 39,19 & drow & 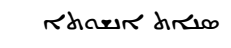 & Rharsiv \\
\hline & & Rharer & & \\
\hline \multirow[t]{2}{*}{$\mu о \lambda v ́ v \omega(-\mathrm{o} \mu \alpha \imath)$} & 39,17 & 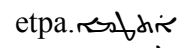 & etpa.sळdir & etpa.sobdir \\
\hline & 40,38 & r.as sti & etpa.rsfdir & etpa.تrikor \\
\hline$\mu o ́ \lambda v \sigma \mu \alpha$ & 40,7 & $\begin{array}{l}\text { pa. } \\
\text { rhantit }\end{array}$ & rhanst & revaf \\
\hline 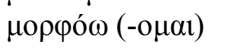 & 39,17 & etpa.sintive & Rhossix & 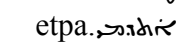 \\
\hline$\mu o ́ \chi \theta$ os & 40,20 & Rhar & rhans & حدلك \\
\hline$\mu \tilde{v} \theta \circ \varsigma$ & 27,9 & $\pi \Delta s$ & & rhan \\
\hline$\mu v \sigma \tau \alpha \gamma \omega \gamma i \alpha$ & 40,11 & 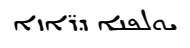 & 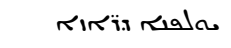 & ${ }^{2}$ rhasirits \\
\hline \multirow{2}{*}{$\mu v ́ \sigma \tau \eta \varsigma$} & 38,11 & حir & & ririrks \\
\hline & 39,2 & & 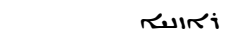 & سiRds \\
\hline$\mu \omega ́ \lambda \omega \psi$ & 39,17 & rastion & rdostio & Rhinv \\
\hline \multirow[t]{3}{*}{ 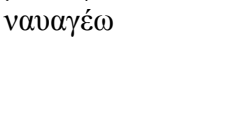 } & 40,11 & ram & 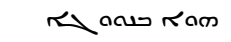 & etpa.> vitr \\
\hline & & 公añ & & \\
\hline & 40,12 & etpa. & etpa. & etpa.入 vidr \\
\hline 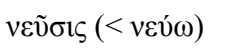 & 38,11 & Rosi & & 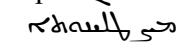 \\
\hline 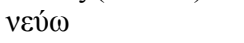 & 27,7 & 心i & & pidr \\
\hline \multirow[t]{3}{*}{ 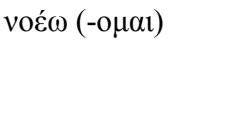 } & 38,11 & rwits & & ristos \\
\hline & 40,5 & rambir & rambir & etpa.لحror \\
\hline & 40,41 & ruamds & ruamds & Lahor \\
\hline vó $\mu \alpha \alpha$ & 40,10 & مصحله & مصحلح & Rharus \\
\hline vo $\mu_{i}^{\prime} \zeta \omega$ & 38,15 & טعב & & af. \\
\hline 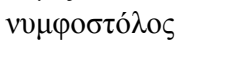 & 40,18 & $\begin{array}{l}\text { कhi } \\
\text { pa.p. }\end{array}$ & pa. p.shi ab & عمعרسك \\
\hline$v \omega \theta \eta \dot{\varsigma}$ & 40,24 & كمنأح & ممن|م & rutas \\
\hline 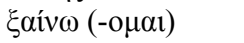 & 39,4 & & حلد & erostir \\
\hline$\xi \dot{\varepsilon} \omega$ & 27,7 & حلa & & si \\
\hline \multirow[t]{2}{*}{$\xi \tilde{\lambda} \lambda \mathrm{ov}$} & 38,12 & معهم & & محلنح \\
\hline & 39,7 & معeمr & معهمr & مصله \\
\hline о̋ $\gamma \kappa о \varsigma$ & 39,16 & Risor & حمכית & حمحتת \\
\hline ódoutrópos & 40,24 & RTOLN & rhal & Rewion \\
\hline
\end{tabular}

${ }^{50}$ Le ms. lit en réalité rhacosss qui doit être considéré comme une erreur pour rhansiss. 


\begin{tabular}{|c|c|c|c|c|}
\hline Grec & $P G$ & S1 & $\mathrm{Sm}$ & S2 \\
\hline 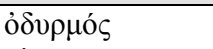 & 39,18 & את. & محلى & Rמw \\
\hline 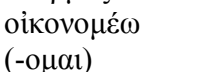 & 38,11 & etpa.urivin & & mistir \\
\hline 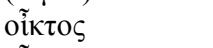 & 40,9 & Ssui & Nowi & Rhoukoones \\
\hline \multirow[t]{2}{*}{ oíos } & 27,4 & prever & & p.p. \\
\hline & 27,8 & pren & & p.p. \\
\hline ӧкvо૬ & 40,31 & rharns & rhasoss & טרושהמא \\
\hline ỏífos & 40,5 & ملفيه & ملفي & ורחור \\
\hline \multirow[t]{2}{*}{$\dot{\delta} \mu 1 \lambda \dot{\varepsilon} \omega$} & 39,10 & 采 & ary & אגרות, \\
\hline & 39,13 & indir & rivir & etpa.ngndir \\
\hline ö $\mu$ otos & 39,16 & כishosis & 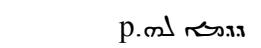 & p.m \\
\hline \multirow[t]{2}{*}{$\dot{\alpha} \mu \mathrm{o} \lambda \mathrm{o} \gamma \dot{i} \alpha$} & 40,8 & R.xace & R.xace & 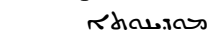 \\
\hline & 40,8 & Rraar & r.xane & 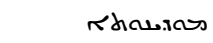 \\
\hline 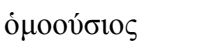 & 40,43 & حi حسك & 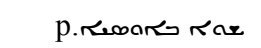 & 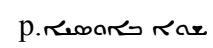 \\
\hline 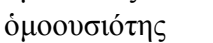 & 40,41 & حi صليم & reoar dione & reoar drones \\
\hline$\dot{\alpha} \mu \omega ́ v v \mu \mathrm{o} \varsigma$ & 39,12 & כi عكى & p.s res & p. pare \\
\hline 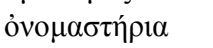 & 40,1 & sce & ressar & राmaser \\
\hline \multirow[t]{3}{*}{ őv $\tau \omega \varsigma$} & 40,12 & 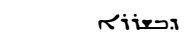 & Rivir & durivir \\
\hline & 40,16 & Ritro & River & durivir \\
\hline & 40,27 & حיivir & Ritirs & durivir \\
\hline ỏ $\hat{u} \varsigma$ & 40,39 & טג & 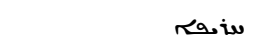 & ملeله \\
\hline 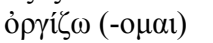 & 40,39 & etpa.dosidir & etpa.dvisidir & $4 i$ \\
\hline 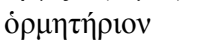 & 39,7 & raru der & حسג & حسd \\
\hline ö $\sigma \tilde{v} \varsigma$ & $40,40^{51}$ & $r 5$ & Rhoor & $r 5$ \\
\hline oủpía & 40,12 & Rhivar Ruai & Rhivar Ruai & Rhum حuai \\
\hline ỏ $\psi \varepsilon ́$ & 40,46 & riware & dorivarss & dorivars \\
\hline \multirow[t]{5}{*}{ ö $\psi 1 \varsigma$} & 27,3 & row & & مس \\
\hline & 27,7 & row & & గLW \\
\hline & 40,6 & row & row & rus \\
\hline & 40,10 & RoLN & row & Rhiw \\
\hline & 40,41 & riev & Riev & Rhiw \\
\hline ő & 40,38 & ssai & حمعلم & حمعلم \\
\hline 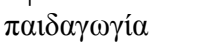 & 40,18 & T. & rhan ac & Rharid \\
\hline$\pi \alpha i ́ \delta \varepsilon v \sigma 1 \varsigma$ & 27,1 & Rhaxiss & & Rhasis \\
\hline$\pi \alpha 1 \delta$ íov & 39,4 & & & rex \\
\hline 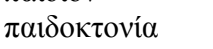 & 38,18 & ملإald عصتم & & مf \\
\hline$\pi \alpha v \eta \gamma v \rho i \zeta \omega$ & 40,1 & No & 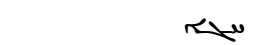 & حגרגי \\
\hline \multirow[t]{2}{*}{$\pi \alpha v \eta ́ \gamma v \rho 1 \varsigma$} & 38,16 & 幽 & & גמורא \\
\hline & 38,17 & "Nح & & Rת \\
\hline 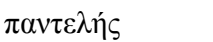 & 40,32 & 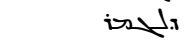 & ris & riser \\
\hline$\pi \alpha ́ v \tau O \tau \varepsilon$ & 27,4 & حلحت & & حصلام \\
\hline$\pi \alpha \rho \alpha ́ \delta \mathrm{o} \xi o \varsigma$ & 40,43 & reen & Rרen ren & rimesh \\
\hline$\pi \alpha \rho \alpha \operatorname{s\varepsilon } \omega$ & 40,18 & sلu & sلي & af.,dire \\
\hline$\pi \alpha \rho \alpha \kappa \alpha \tau \alpha \theta \eta \dot{\kappa} \kappa \eta$ & 40,41 & rotia & rotis & حمحلה \\
\hline$\pi \alpha \rho \alpha \lambda \alpha \mu \beta \alpha ́ v \omega$ & 40,30 & ع & ل & נסבר \\
\hline$\pi \alpha \rho \alpha \pi \lambda \eta ́ \sigma 10 \varsigma$ & 40,30 & مiٔصح & مأحسم & p.r.s \\
\hline$\pi \alpha \rho \alpha \tau \varepsilon i v \omega$ & 40,29 & ustos & pa.,msi & pa.,mdi \\
\hline 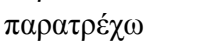 & 40,14 & tmi & tmi & \\
\hline \multirow{2}{*}{$\pi \alpha ́ \rho \varepsilon 1 \mu \mathrm{t}(<\varepsilon i ̉ \mu i ́)$} & $40,12^{52}$ & مחס & مחק & مiٔد \\
\hline & 40,46 & 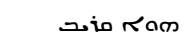 & 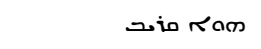 & דע.pa \\
\hline$\pi \alpha \rho \varepsilon \mu \pi i \pi \tau \omega$ & 40,26 & las & las & 2.4 \\
\hline$\pi \alpha \rho \varepsilon ́ \rho \chi о \mu \alpha \imath$ & 39,14 & حصi & حרi & סה:ס \\
\hline$\pi \alpha \operatorname{sí} \mu 1(-\varepsilon v \alpha \imath)$ & 40,33 & R.1. & Rres & STrer \\
\hline$\pi \alpha ́ \rho \varepsilon \rho \gamma о \varsigma(-\omega \varsigma)$ & 40,1 & כמשעת & במשתרא & durnas \\
\hline$\pi \alpha \rho о \rho \alpha ́ \omega$ & 39,13 & حم) & תم & 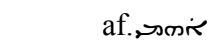 \\
\hline \multirow[t]{3}{*}{$\pi \alpha ́ \sigma \chi \alpha$} & 40,24 & ولانجم & Q & ren \\
\hline & 40,30 & هُ & م & बת \\
\hline & 40,30 & هُ & هلأحم & ف5 \\
\hline
\end{tabular}

${ }^{51}$ Mais juste un peu plus haut en 40,40, les trois versions lisent r roem.

${ }^{52}$ Mais en 40,12 juste après, les trois versions ont 


\begin{tabular}{|c|c|c|c|c|}
\hline Grec & $P G$ & $\mathrm{~S} 1$ & $\mathrm{Sm}$ & $\mathrm{S} 2$ \\
\hline & 40,40 & र & र & $\mathrm{Ru}_{5} \mathrm{~g}$ \\
\hline \multirow[t]{7}{*}{$\pi \alpha ́ \sigma \chi \omega$} & 27,6 & ․ㄴ & & 20 \\
\hline & 39,4 & & .4 & 20 \\
\hline & 39,9 & 2.4 & 20 & 20 \\
\hline & 39,9 & 5.4 & 20 & 20 \\
\hline & 39,18 & 5.4 & zo & 20 \\
\hline & 40,16 & $=4$ & 20 & 20 \\
\hline & 40,25 & 2 & 2.4 & 20 \\
\hline \multirow[t]{3}{*}{ 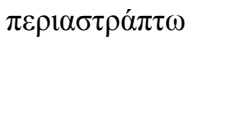 } & 40,6 & af. & af. & af.hal \\
\hline & 40,6 & af. & af. & af.hal \\
\hline & 40,36 & af.imsin & af. & af.د \\
\hline $\begin{array}{l}\pi \varepsilon \rho \iota \beta о \mu \beta \varepsilon \dot{\varepsilon} \omega \\
(-\mathrm{o} \mu \alpha)\end{array}$ & 27,2 & & & rasin \\
\hline$\pi \varepsilon \rho i ́ \varepsilon \rho \gamma о \varsigma$ & 40,37 & דתمרת| & תרمحת| & خمم.pa.p.p \\
\hline$\pi \varepsilon \rho 1 \lambda \alpha \mu \beta \alpha ́ v \omega$ & 39,18 & عمل & عمل & טבת \\
\hline $\begin{array}{l}\pi \varepsilon \rho \imath \lambda \dot{\alpha} \mu \pi \omega \\
(-o \mu \alpha \imath)\end{array}$ & 40,41 & $\begin{array}{r}\text { ustsis } \\
\text { etpa. }\end{array}$ & etpa.urstis & etpa.imstir \\
\hline \multirow[t]{2}{*}{$\pi \varepsilon \rho i ́ o \delta o \varsigma$} & 27,10 & حمiֹ תم & & Rhouivds \\
\hline & 40,5 & חת & مाل & 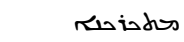 \\
\hline 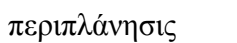 & 40,35 & rhout & rhant & గms \\
\hline$\pi \varepsilon \rho 1 \sigma \pi \mathrm{o} \delta \delta \alpha \sigma \tau \mathrm{s}$ & 39,10 & ra & م & 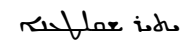 \\
\hline$\pi \varepsilon \rho 1 \varphi о \rho \alpha ́$ & 40,15 & 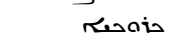 & حسمحسم & ๘m \\
\hline$\pi \mathrm{\imath} \zeta \zeta \omega(-o \mu \alpha \mathrm{\imath})$ & 40,27 & ك & 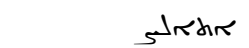 & stir \\
\hline$\pi \imath \theta \alpha \nu$ ó ${ }^{\prime} \varsigma$ & 39,3 & & గa. & रमेon.as \\
\hline$\pi \mu \varepsilon \lambda \eta ́$ & 40,40 & rit & roid & טلصم \\
\hline \multirow[t]{2}{*}{$\pi \lambda \alpha v \alpha ́ \omega$} & 39,4 & & rb & राल \\
\hline & 40,35 & ab & $\pi$ & बחת \\
\hline$\pi \lambda \alpha ́ v \eta$ & 39,8 & rhant & rhant & t) \\
\hline$\pi \lambda \alpha \sigma \tau$ ó $\varsigma$ & 40,16 & Sסודיה & תוש:דים & 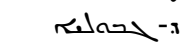 \\
\hline 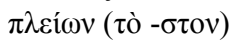 & 40,40 & గr人 & مس حس & rharel. \\
\hline$\pi \lambda \varepsilon o v \varepsilon \xi i ́ \alpha$ & 39,19 & रमner & रhan & Rharale \\
\hline$\pi \lambda \tilde{\eta} \theta 0 \varsigma$ & 40,29 & حهs & حهs & حיזr \\
\hline$\pi \lambda \eta ́ \rho \omega \sigma \iota \varsigma$ & 39,8 & rexar & Sartar & ras \\
\hline$\pi \lambda$ óos & 39,14 & 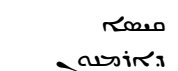 & rant & rant \\
\hline \multirow[t]{5}{*}{$\pi$ ó $\theta$ os } & 38,10 & טחבא & & Rhowi \\
\hline & 39,8 & טחבא & טחבת & Rhoswi \\
\hline & 40,12 & טمحת & טمحת & Rhowi \\
\hline & 40,36 & טחحת & טمحת & Rhoswi \\
\hline & 40,26 & טمحת & טמבת & Rhosui \\
\hline$\pi \mathrm{ol \varepsilon ́} \omega(-\mathrm{o} \mu \alpha \imath)$ & 40,38 & rom & ridr & אוdרוז \\
\hline$\pi \mathrm{o} \lambda \varepsilon \mu \varepsilon \dot{\varepsilon} \omega$ & 40,16 & etpa.sdidir & 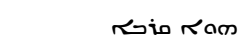 & rioditir \\
\hline$\pi \mathrm{o} \lambda \varepsilon \dot{\mu} \mu \mathrm{L} \sigma$ & 40,37 & revdados & risdados & 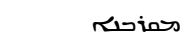 \\
\hline$\pi \mathrm{o} \lambda v \varepsilon 1 \delta \eta \dot{\varsigma}$ & 40,4 & $\begin{array}{l}\text { Rhaneo } \\
\text { Rhasiar }\end{array}$ & Rharis Rhan & 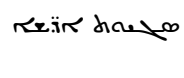 \\
\hline$\pi \mathrm{o} \lambda v \tau \varepsilon \dot{\lambda} \overline{\varepsilon l \alpha}$ & 38,11 & rhaies & & rition \\
\hline 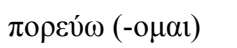 & 39,19 & pa.npin & pa.n\க் & Sir \\
\hline $\begin{array}{l}\pi \rho o ́ \sigma \omega \\
(\pi \circ \rho \rho \omega \tau \varepsilon \dot{\varepsilon} \rho \omega)\end{array}$ & 38,10 & 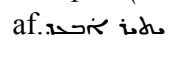 & & ravail ish \\
\hline$\pi \rho \tilde{\alpha} \gamma \mu \alpha$ & 40,14 & 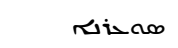 & مهiح & rhios \\
\hline$\pi \rho \alpha ́ \sigma \sigma \omega$ & 40,22 & حרו. & حרו: & iso \\
\hline$\pi \rho \varepsilon \sigma \beta \varepsilon v ́ \omega$ & 27,5 & af. & & غخصe.pa \\
\hline \multirow[t]{2}{*}{$\pi \rho \circ \beta \alpha ́ \alpha \lambda \lambda \omega$} & $40,10^{53}$ & فiت.pa & فiات.pa & af.wion \\
\hline & 40,16 & af. منوم & af. & af.wion \\
\hline \multirow[t]{2}{*}{$\pi \rho \circ \varepsilon ́ \chi \omega$} & 39,14 & ins & ins & pa.idi \\
\hline & 40,26 & Rivar & خلر.pa.p.p & خلر.pa.p.p \\
\hline$\pi \rho o ́ \theta v \rho o v$ & 40,16 & 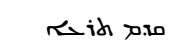 & 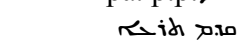 & रac \\
\hline$\pi \rho о \kappa \alpha ́ \mu \nu \omega$ & 40,20 & ف. pa., pa & 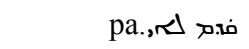 & pa.pa حמל \\
\hline 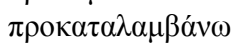 & 40,15 & م.7 مس. & 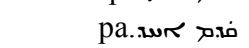 & pa.n \\
\hline
\end{tabular}

${ }^{53}$ Mais en 40,10, trois lignes plus haut, les trois versions ont wior. 


\begin{tabular}{|c|c|c|c|c|}
\hline Grec & $P G$ & $\mathrm{~S} 1$ & $\mathrm{Sm}$ & $\mathrm{S} 2$ \\
\hline$\pi \rho \circ \sigma \alpha ́ \gamma \omega$ & 40,12 & af.גحس & af.」ح & فiات.pa \\
\hline 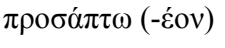 & 38,12 & 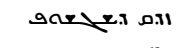 & & 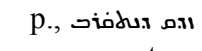 \\
\hline & & p. & & etpa. \\
\hline \multirow[t]{4}{*}{$\pi \rho о \sigma \beta \alpha ́ \alpha \lambda \lambda \omega$} & 39,13 & غxلدpa & $\infty$ & on \\
\hline & $40,10^{54}$ & etpa._ibin & غ.د.Ja & غ.دpa. \\
\hline & 40,10 & 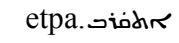 & pa.」 & غ غ.ل. \\
\hline & 40,35 & אRלفi._etpa & 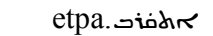 & غ.ل.ja \\
\hline$\pi \rho о \sigma \beta о \lambda \eta ́$ & 40,28 & ممتحه? & $\nabla x$ & 9 \\
\hline \multirow[t]{2}{*}{ 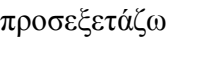 } & 27,8 & 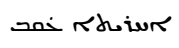 & & af., منهa خمد \\
\hline & & pa. & & pa. \\
\hline 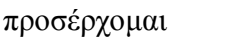 & 40,31 & inv & ino & תitoti \\
\hline$\pi \rho о \sigma \kappa \alpha \kappa о \pi \alpha \theta \varepsilon \dot{\varepsilon} \omega$ & 39,17 & حدل & حدل & etpa.sibdir \\
\hline$\pi \rho о \sigma \kappa v \alpha ́ o \mu \alpha \imath$ & 27,1 & nev & & ع \\
\hline \multirow[t]{2}{*}{ 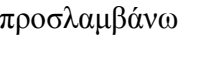 } & 39,13 & عمل & عمل & נow \\
\hline & 40,31 & נمد & נסבר & ملم \\
\hline \multirow[t]{2}{*}{$\pi \rho \circ \sigma о \mu \imath \lambda \dot{\varepsilon} \omega$} & 39,9 & pa.dلـ & תhror & 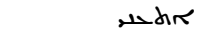 \\
\hline & 39,9 & pa.dله & مR' & ملهר \\
\hline 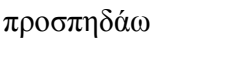 & 40,31 & 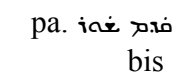 & pa.ini & smikor \\
\hline$\pi \rho \circ \sigma \pi i ́ \pi \tau \omega$ & 40,31 & فــا pa. sic & ف & ${ }^{55} \mathrm{w}$ iो \\
\hline$\pi \rho о \sigma \rho і ́ \pi \tau \omega$ & 40,31 & las & las & si \\
\hline$\pi \rho о \sigma \tau \alpha ́ \sigma ı \varsigma \varsigma$ & 40,41 & مersar & مשתמא & مest حتصوم \\
\hline$\pi \rho \circ \tau \varepsilon \lambda \varepsilon \dot{\varepsilon} \omega$ & 39,10 & איR & drster & etpa.ıirir \\
\hline \multirow[t]{2}{*}{ 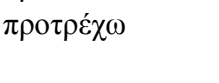 } & $39,15^{56}$ & תidis & foidir gidir & 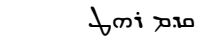 \\
\hline & & etpa.rfinis & etpa., etpe. & \\
\hline 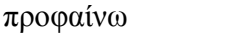 & 39,10 & pa.ini & pa.inis & pa.rim \\
\hline \multirow[t]{2}{*}{$\pi \rho \omega \tau$ бó } & $40,6^{1}$ & 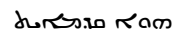 & م.דתה & حרטי مודדית \\
\hline & $40,6^{2}$ & 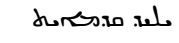 & م.דתיא & 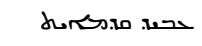 \\
\hline$\pi \rho \tilde{\omega} \tau о \varsigma$ & 38,9 & مוגדיم & & Reri \\
\hline$(-\tau o v)$ & 27,7 & 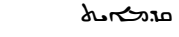 & & (1.0 \\
\hline \multirow[t]{2}{*}{$\pi \tau \omega \chi \varepsilon v ́ \omega$} & 40,31 & i.w & ram ras & sam ram \\
\hline & 40,34 & ine & i.wo & peostir \\
\hline$\pi v \rho o ́ \omega(-о \mu \alpha \imath)$ & 40,10 & مم: & مم: & ples \\
\hline$\pi v ́ \rho \omega \sigma ı \varsigma$ & 40,9 & مم:ته & ماست & כחمית \\
\hline jó $\delta 10 \varsigma(-\omega \varsigma)$ & 40,19 & durdedx & hurdedx & حلد \\
\hline$\dot{\rho} \alpha \nu \tau^{\prime} \zeta \omega(-o \mu \alpha \imath)$ & 40,19 & eopitir & eocitir & tobtir \\
\hline 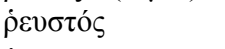 & 39,18 & R.uer & 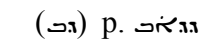 & 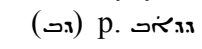 \\
\hline 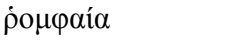 & 39,16 & rusai & rusai & مiv \\
\hline 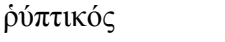 & 40,11 & af. pr. & af. p.حזع. & p.ois \\
\hline$\sigma \alpha \theta$ pó $\varsigma$ & 27,5 & נחספר & & ملفلح \\
\hline \multirow[t]{2}{*}{$\sigma \alpha ́ \rho \kappa \omega \sigma ı \varsigma^{57}$} & 38,3 & & & Rhouiarbss \\
\hline & 40,2 & Rhoritas & Rhowioshos & Rhouiashss \\
\hline$\sigma \alpha \varphi \eta ́ \varsigma(-\tilde{\omega} \varsigma)$ & 40,10 & גorist & duraly & dored \\
\hline 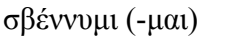 & 40,10 & etpa. & etpa. & $n \rightarrow x$ \\
\hline 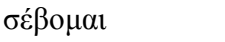 & 39,6 & etpa. אidud & etpa. ridir & etpa. ulatir \\
\hline 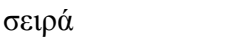 & 40,33 & ? Rhin & rivo & م R \\
\hline$\sigma \varepsilon 1 \sigma \mu o ́ s$ & 40,14 & iٓحله & iانح & ral \\
\hline$\sigma \eta \mu \varepsilon i \omega \sigma ı \varsigma$ & 40,4 & Ssrai & Norai & rhr \\
\hline$\sigma \iota \omega \pi \dot{~}$ & 39,5 & & rotar & roter \\
\hline 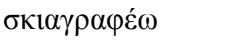 & 40,6 & 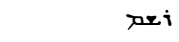 & i i & 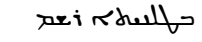 \\
\hline
\end{tabular}

${ }^{54}$ Mais en 40,10, quelques lignes plus loin, les trois versions lisent غx pa.

${ }^{55}$ Dans l'édition du Discours 40 (p. 141, 1. 39), nous avons édité par erreur iो iो

${ }^{56}$ Mais en 39,15, à la ligne précédente, les trois versions lisent مro im

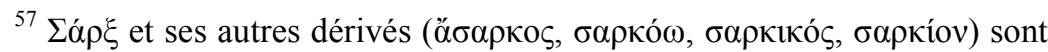

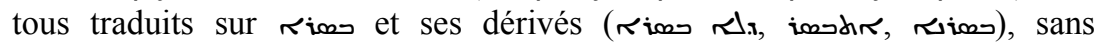
qu'aucune particularité ne puisse être relevée. 


\begin{tabular}{|c|c|c|c|c|}
\hline Grec & $P G$ & $\mathrm{~S} 1$ & $\mathrm{Sm}$ & $\mathrm{S} 2$ \\
\hline 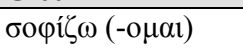 & 40,32 & etpa. & etpa. & Ram Sam \\
\hline$\sigma o ́ \varphi 1 \sigma \mu \alpha$ & 39,5 & & ruas & טمحكה \\
\hline \multirow[t]{2}{*}{ бофıбти́s } & 27,1 & स6.000 & & תדמחרטית \\
\hline & 40,102 & תטרמהת & תחבתות & pa.p. \\
\hline блóvios & 39,14 & لحس & relex & Nel. \\
\hline 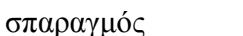 & 39,5 & & مחה:مr & rextios \\
\hline$\sigma \pi \alpha \rho \alpha ́ \sigma \sigma \omega$ & 27,6 & pa. & & حרת \\
\hline$\sigma \pi \imath v \theta \eta ́ \rho$ & 27,5 & Rhioss & & 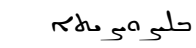 \\
\hline$\sigma \pi \lambda \alpha ́ \gamma \chi \nu \circ \nu(-\alpha)$ & 27,2 & تصنم & & Ẽن \\
\hline$\sigma \pi 0 v \delta \alpha ́ \zeta \omega$ & $40,22^{2}$ & 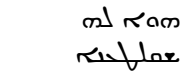 & 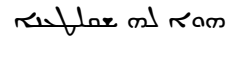 & 9 \\
\hline \multirow[t]{2}{*}{ 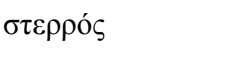 } & 27,3 & pa.p.p. غ غ & & حسer \\
\hline & 40,6 & pa. p.p. غi & معنج & مrer \\
\hline$\sigma \tau$ oııєı́́ $\omega$ & 39,8 & pa. , غ غ & pa. , iغ & etpa.cafbir \\
\hline$\sigma \tau \mathrm{o} \lambda \dot{n}$ & 40,17 & لحمعك & لصمعr & सل \\
\hline 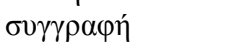 & 40,31 & rata & roda & ribr \\
\hline $\begin{array}{l}\sigma v \gamma \kappa \alpha \lambda \hat{\pi} \pi \tau \omega \\
(-о \mu \alpha \iota)\end{array}$ & 38,8 & etpa. & & etpa. תividir \\
\hline$\sigma 0 \gamma \kappa \lambda \varepsilon 1 \sigma \mu \sigma^{\prime} \varsigma$ & 40,24 & טרחסיא & טרחعשא & reaii \\
\hline$\sigma v \zeta \varepsilon v ́ \gamma \nu v \mu 1$ & 40,30 & ט.t. & ح. & pa. $\searrow_{a i}$ \\
\hline 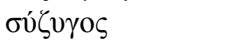 & 40,36 & حi سنم & حi سنم & حi וه \\
\hline бטко & 39,9 & Rhiserg Rhid & Rhmeng Rhid & عمدى \\
\hline$\sigma v \mu \beta \alpha i ́ v \omega$ & 27,5 & علم & & etpa. rorisir \\
\hline$\sigma u ́ \mu \mu \varepsilon \tau \rho \circ \varsigma$ & 40,6 & Rhwarss & 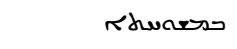 & pa.p.p. לעu \\
\hline$\sigma v \mu \pi \alpha v \eta \gamma v \rho i \zeta \zeta \omega$ & 38,17 & pa. حم نـي & & 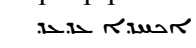 \\
\hline$\sigma v \mu \pi \alpha \rho \alpha \lambda \alpha \mu \beta \alpha ́ v \omega$ & 40,19 & river & 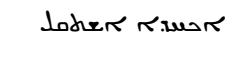 & Rحuidor \\
\hline $\begin{array}{l}(-\mathrm{o} \mu \alpha \mathrm{l}) \\
\sigma \nu \mu \pi i \pi \tau \omega\end{array}$ & 40,28 & Jas & Jas & $\therefore$.4 \\
\hline $\begin{array}{l}\sigma 0 \mu \pi \lambda \eta \rho o ́ \omega \\
(-о \mu \alpha \imath)\end{array}$ & 38,9 & $\operatorname{irs}$ din & & 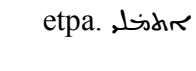 \\
\hline$\sigma 0 \mu \pi \lambda \eta ́ \rho \omega \sigma ı \varsigma$ & 38,10 & Rastrar & & rasos \\
\hline \multirow[t]{2}{*}{$\sigma \hat{\mu} \mu \pi \tau \omega \mu \alpha$} & 40,14 & rulas & rilas & p. . \\
\hline & 40,15 & rillas & rithas & 年 \\
\hline 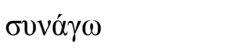 & 40,45 & خع. pa & خد. pa & af. \\
\hline 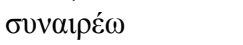 & 40,41 & נסב אתטורא & נסב ארטוזר & خע.pa \\
\hline$\sigma v v \alpha ́ \lambda \lambda \alpha \gamma \mu \alpha$ & 40,27 & ชูin & rahow/rain & ralow \\
\hline 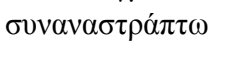 & 39,14 & 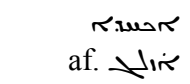 & 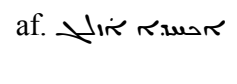 & 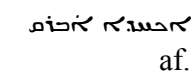 \\
\hline$\sigma v v \alpha v \varepsilon \dot{\rho} \chi 0 \mu \alpha 1$ & 40,2 & Ris & Rii & Rin \\
\hline бvvó $\pi \tau \omega$ & 38,15 & pa. pas & & af. halk \\
\hline $\begin{array}{l}\sigma v v \alpha \rho \mu \mathrm{o} \lambda \mathrm{o} \gamma \dot{\varepsilon} \omega \\
(-\mathrm{o} \mu \alpha)\end{array}$ & 40,39 & etpa. & etpa. & etpa. \\
\hline 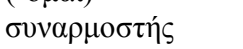 & 40,18 & S19 הית & 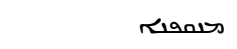 & 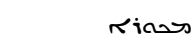 \\
\hline$\sigma v v \alpha \rho \pi \alpha \dot{\zeta} \omega$ & 27,2 & abv & & ع ra \\
\hline 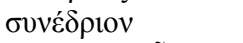 & 27,9 & ratios & & 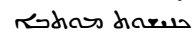 \\
\hline 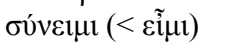 & 38,8 & 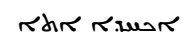 & & etpa. \\
\hline 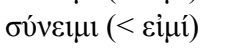 & 40,46 & R.tur Ram & rimer ram & مam \\
\hline 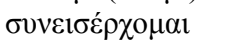 & 40,46 & 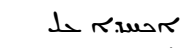 & محسוזم حل & حل حم) \\
\hline 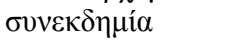 & 40,3 & rhal & rhal & Rrotw \\
\hline 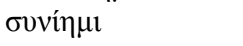 & 40,28 & هוح & הוح & etpa. لـhoor \\
\hline бvvó $\mu 1 \lambda \circ \varsigma$ & 40,18 & p. pilis & p. pilis & כi حسبح \\
\hline 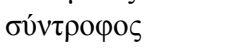 & 27,2 & حم & & whro \\
\hline 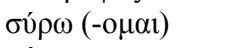 & 39,9 & ת & $\mu$ is & ari \\
\hline$\sigma u ́ \sigma \tau \eta \mu \alpha$ & 38,10 & 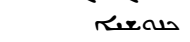 & & مחمتر \\
\hline$\sigma \varphi \varepsilon v \delta o v \alpha ́ \omega$ & 40,43 & كسم حملهم & & pa.pa \\
\hline$\sigma \varphi \rho \alpha \gamma i \zeta \omega$ & 40,15 & i i & i i & - \\
\hline$(-o \mu \alpha \imath)$ & 40,18 & peritur & pridir & phutir \\
\hline$\sigma \varphi \rho \alpha \gamma i ́ \varsigma$ & 40,18 & Nsrai & Nscai & rab \\
\hline$\sigma \chi 0 \lambda \alpha ́ \zeta \omega$ & 27,3 & 凹 & & sritir \\
\hline$\sigma \omega ́ \zeta \omega(-o \mu \alpha \imath)$ & 40,19 & etpa. Vlasir & mader/Vadire & Glaterer \\
\hline
\end{tabular}




\begin{tabular}{|c|c|c|c|c|}
\hline Grec & $P G$ & $\mathrm{~S} 1$ & $\mathrm{Sm}$ & $\mathrm{S} 2$ \\
\hline \multirow[t]{15}{*}{$\sigma \tilde{\omega} \mu \alpha$} & 27,3 & Exan & & riva \\
\hline & 27,3 & لحمعري & & Riva \\
\hline & $27,6^{58}$ & لحمعرs & & riva \\
\hline & 27,10 & ה & & riva \\
\hline & 39,9 & הars & ssran & Riva \\
\hline & 40,1 & הזה & لحمrs & riva \\
\hline & 40,11 & המה & המה & riva \\
\hline & 40,11 & stal & stral & riva \\
\hline & 40,11 & sseal & sard & Riva \\
\hline & 40,13 & scal & stal & Rivo \\
\hline & 40,26 & sseal & (s) & Riva \\
\hline & 40,33 & Nar) & (s) & Riva \\
\hline & 40,39 & הars & لحمrs & Riva \\
\hline & 40,40 & stan & لحمrst & Riva \\
\hline & 40,45 & stan & sact & रा \\
\hline$\sigma \omega \mu \alpha \tau \iota \kappa o ́ \varsigma$ & 39,14 & 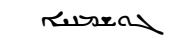 & 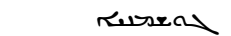 & rutin \\
\hline$(-\tilde{\omega} \zeta)$ & 39,16 & hureseal & horiva & hursensen \\
\hline \multirow[t]{2}{*}{$\sigma \omega ́ \varphi \rho \omega \nu$} & 39,14 & תav & תרפא & טיבת \\
\hline & 40,19 & טבהצה & טבהצה & rav \\
\hline$\tau \alpha \lambda \alpha \nu \tau \varepsilon v ́ \omega$ & 40,42 & Joh & Joh & pa. فممج \\
\hline$\tau \alpha \mu{ }^{\prime} \alpha \varsigma$ & 40,29 & ria & riat & 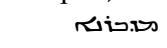 \\
\hline \multirow[t]{2}{*}{$\tau \alpha \pi \varepsilon 1 v o ́ \tau\rceil \varsigma$} & 38,11 & Rhounss & & 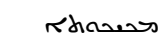 \\
\hline & 39,13 & Rhangar & Salions & תחمحת \\
\hline$\tau \alpha \pi \varepsilon i ́ v \omega \sigma ı \varsigma$ & 39,17 & נתחحת & תחתרז & 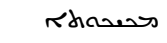 \\
\hline$\tau \varepsilon \imath \chi i \zeta \omega(-o \mu \alpha \imath)$ & 40,10 & riar hor & rine dur & pa.p.p. نهم \\
\hline \multirow[t]{2}{*}{$\tau \varepsilon \lambda \varepsilon 10 \pi$ o'ó $\varsigma$} & 40,26 & حרו: & p. Rhaius/ & 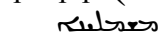 \\
\hline & & p. Rhaiss & & \\
\hline \multirow[t]{3}{*}{$\tau \varepsilon ́ \lambda \varepsilon \varepsilon 10 \varsigma$} & 38,12 & riss & & תמשליה \\
\hline & 40,35 & durabses & גarabses & rises \\
\hline & 40,40 & riss & riss & Serstris \\
\hline ( & 40,44 & rhaies & rhaies & Rhoulars \\
\hline$(\tau \varepsilon \lambda \varepsilon i ́ \omega \varsigma)$ & 40,27 & doriss & hories & strabses \\
\hline$\left(\tau \varepsilon \lambda \varepsilon^{\prime} \omega \varsigma\right)$ & 40,28 & hories & horist & duratses \\
\hline$\tau \varepsilon \lambda \varepsilon 10 ́ \tau \eta \varsigma$ & 39,14 & Rhaies & Rhaies & Rhoulars \\
\hline$\tau \varepsilon \lambda \varepsilon i ́ \omega \sigma i \varsigma$ & 40,18 & rhaies & rhaies & عatsor \\
\hline \multirow[t]{2}{*}{$\tau \varepsilon \lambda \varepsilon \varepsilon \tau \eta ́$} & 39,4 & & rhesed & RाRi \\
\hline & 39,6 & & Rhesed & RाRi \\
\hline 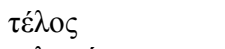 & 40,24 & rastar & rar & مى م \\
\hline$\tau \varepsilon \lambda \omega \nu \varepsilon \dot{\varepsilon} \omega$ & 39,9 & pers & pass & خع pa.p \\
\hline$\tau \varepsilon ́ \mu \nu \omega$ & 40,7 & טLم & SW & همم \\
\hline$\tau \varepsilon \rho \alpha \tau \varepsilon i ́ \alpha$ & 27,8 & ris & & כו. R \\
\hline$\tau \varepsilon \rho \alpha \tau \varepsilon v ́ o \mu \alpha l$ & 27,10 & 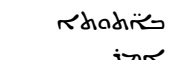 & & כוגא \\
\hline$\tau \varepsilon \rho \pi v o ́ \varsigma$ ( $\tau$ ò -óv) & 40,20 & रमcon & रhorag & rharewm \\
\hline$\tau \tilde{\eta} \xi 1 \varsigma$ & 40,9 & Rar & عar & הם הרא \\
\hline \multirow[t]{2}{*}{$\tau \eta ি \rho \eta \sigma 1 \varsigma$} & $39,8^{1}$ & rhiat & rhiat & Rhaiat \\
\hline & $39,8^{2}$ & Rhiats & rhiat & Rhaint \\
\hline$\tau \rho \alpha v o ́ \varsigma$ & 39,20 & حلdrar & حلerat & 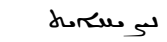 \\
\hline$\tau \rho \alpha \tilde{\mu} \mu \alpha$ & 40,31 & Rhalos & Rhalas & rhans \\
\hline$\tau \rho \alpha v \mu \alpha \tau i ́ \alpha \varsigma$ & 40,31 & pa.p.p. a & pa. p.p. a & pa.p.p.p \\
\hline$\tau \rho о \varphi \eta ́ ~$ & 38,12 & ribaras & & rheoid \\
\hline$\tau \rho v \varphi \alpha ́ \omega$ & 39,20 & $\begin{array}{l}\text { rooidir } \\
\text { etpa. }\end{array}$ & 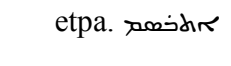 & pa. خمة \\
\hline$\tau v \gamma \chi \alpha \dot{\alpha} v \omega$ & 39,7 & $\leq x$ & $x .4$ & ,aterer \\
\hline \multirow[t]{6}{*}{ 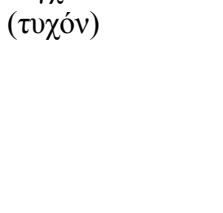 } & 39,14 & 2.4 & 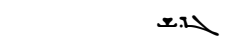 & حصi \\
\hline & 39,18 & 2.4 & 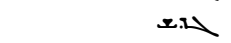 & אזحט \\
\hline & 40,12 & $=2$ & $=2$ & , adure \\
\hline & 40,19 & 5.4 & 5.4 & ,ature \\
\hline & 40,20 & 5.4 & 2.4 & ,aterer \\
\hline & 40,21 & ram & ram & אater \\
\hline
\end{tabular}

${ }^{58}$ Mais en 27,7, S1 et S2 ont م مون. 


\begin{tabular}{|c|c|c|c|c|}
\hline Grec & $P G$ & $\mathrm{~S} 1$ & $\mathrm{Sm}$ & $\mathrm{S} 2$ \\
\hline & 40,22 & ․ㄴ & 2.4 & ,ader \\
\hline & 40,22 & 5.4 & 5 & , adser \\
\hline & 40,22 & $=4$ & 5.4 & , aderer \\
\hline & 40,23 & $=4$ & 5 & odurer \\
\hline & 40,27 & $=4$ & $=4$ & rater \\
\hline & 40,27 & ram & ram & other \\
\hline & 40,26 & 2.4 & 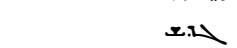 & ,ater \\
\hline & 40,31 & 2.4 & 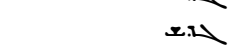 & ,ater \\
\hline & 40,31 & $=$ & $=$ & ,ater \\
\hline & 40,34 & $=4$ & $=4$ & Rever \\
\hline & 40,34 & 5 & 5.4 & ,aber \\
\hline$\tau v \rho \alpha v v \varepsilon \dot{\varepsilon} \omega(-о \mu \alpha \imath)$ & 40,24 & etpa. xisidir & etpa. & محर \\
\hline 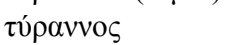 & 39,4 & & Rxaiss & raib \\
\hline$\tau \tilde{v} \varphi \circ \varsigma$ & 39,18 & Rhasi & Rhasi & Rhalars \\
\hline$v ๊ \lambda \eta$ & 27,2 & vam & & sمals \\
\hline 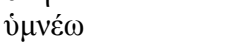 & 38,17 & pa. isi & & pa. ل山க் \\
\hline$\dot{v} \mu \nu \omega \delta i ́ \alpha$ & 40,46 & مחلodr & rhünds & Raver \\
\hline 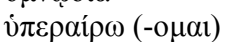 & 40,39 & pisidir & riר & حרi \\
\hline vं $\varepsilon \rho \alpha v \alpha \beta \alpha i v \omega$ & 39,8 & حصi لد & ل & مأخل, لد \\
\hline vं $\pi \varepsilon \rho \alpha \sigma \pi \hat{\zeta} \omega$ & 40,18 & مחم טلa & ممر vلa & $\begin{array}{r}\text { etpa. } \\
\text { pa. ino }\end{array}$ \\
\hline vं $\pi \varepsilon \rho \beta \alpha ́ \lambda \lambda \lambda \omega$ & 39,6 & etpa. ididir & judidir & منحل \\
\hline vं $\pi \varepsilon \rho \beta o \lambda \eta ́$ & 40,41 & rhoi & rhoi & rhoules \\
\hline 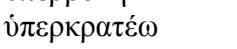 & 40,10 & لح & لحn & حله \\
\hline 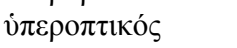 & 40,43 & p. i i & p. & pa.p.p. غمل \\
\hline vं $\pi v o ́ \omega$ & 40,15 & عרב & ل & 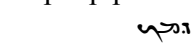 \\
\hline 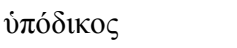 & 40,8 & res dewh & r.is dewd & pa.p.p. טיב \\
\hline 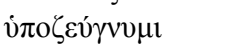 & 27,7 & שרבו & & ح. \\
\hline 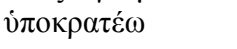 & 40,21 & لحn & pa. & حله \\
\hline 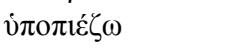 & 27,7 & خבع pa. & & pa. i \\
\hline і் & 40,11 & रमार $\Delta x$, m & रमार $\Delta x, \dot{x}$ & rhudinsl, ms \\
\hline 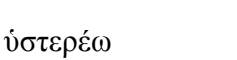 & 40,46 & worder & swader & $\begin{array}{r}\text { pa. } \\
\Sigma 0 \text { Q }\end{array}$ \\
\hline \multirow[t]{2}{*}{ 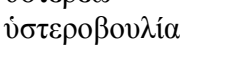 } & 40,24 & Rharus & Rhitur Rharus & 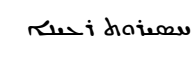 \\
\hline & & Rhiur & & \\
\hline vँ $\varphi \varepsilon \sigma ı \varsigma$ & 40,41 & Rin & |حin & rhas \\
\hline \multirow{2}{*}{ 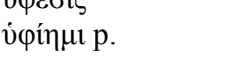 } & 40,22 & عרم & S & Sis \\
\hline & 40,22 & عרم & כ & ح \\
\hline \multirow[t]{3}{*}{ 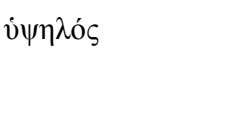 } & $27,9^{1}$ & si & & תلה \\
\hline & $27,9^{2}$ & si & & sلs \\
\hline & 39,18 & Rhasi & rhoulas & rhoules \\
\hline \multirow[t]{2}{*}{ 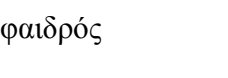 } & 40,46 & గ几 & बת & Rus 5 \\
\hline & 40,46 & 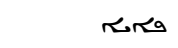 & 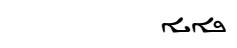 & Sus \\
\hline \multirow[t]{3}{*}{ 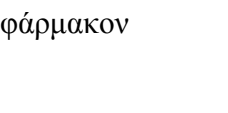 } & 40,12 & حمir & حمiٔم & Non \\
\hline & 40,12 & حمi م & حمiٔم & sso \\
\hline & 40,14 & حمir & حمiٔم & sos \\
\hline$\varphi \alpha ́ \sigma \mu \alpha$ & 39,5 & & هلبك & rhat \\
\hline & 40,14 & וحمin & ו ו & ح \\
\hline$\varphi \varepsilon^{\prime} \gamma \gamma о \varsigma$ & 38,11 & الفم & & الحم \\
\hline$\varphi \varepsilon \imath \delta \omega \lambda i ́ \alpha$ & 40,35 & Rhoushooses & rhashooness & مhafu \\
\hline \multirow[t]{3}{*}{ } & 40,12 & حid & حiأم & אתדו. \\
\hline & 40,12 & حأه & حiأم & אתדה. \\
\hline & 40,27 & حiم & حiأم & af. \\
\hline$\varphi \theta \dot{\varepsilon} \gamma \gamma о \mu \alpha \iota$ & 39,17 & pa. sخ & pa. sخ & Rת \\
\hline \multirow[t]{2}{*}{$\varphi 1 \lambda \alpha v \delta \rho i ́ \alpha$} & 27,7 & dosui & & i \\
\hline & & Rhainel & & \\
\hline$\varphi 1 \lambda \varepsilon^{\prime} \omega$ & 40,4 & i וטבק & i iva & af. \\
\hline 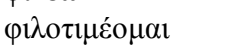 & 38,11 & i iva مoمi R & & etpa. inidir \\
\hline$\varphi \imath \lambda о \tau \imath \mu i^{\prime} \alpha$ & 27,7 & Rinor doswi & & rusar doswi \\
\hline \multirow[t]{2}{*}{$\varphi 1 \lambda \tau^{\tau} \tau \mu \mathrm{s}$} & 27,9 & Rinor doswi & & p. sodic \\
\hline & 27,10 & Rinosuid dow & & Rusce dosvi \\
\hline$\varphi \lambda{ }^{\prime} \gamma \dot{i} \zeta \omega$ & 40,36 & Jin & Jin & af. \\
\hline$\varphi \lambda$ ó $\gamma \mathrm{ivos}$ & 39,16 & xicus & Rin & Rin \\
\hline$\varphi \lambda \operatorname{có}^{\prime} \xi$ & 27,5 & טב.p & & ralin \\
\hline фора́ & 38,8 & ritiss & & Rh.ivs \\
\hline
\end{tabular}




\begin{tabular}{|c|c|c|c|c|}
\hline Grec & $P G$ & $\mathrm{~S} 1$ & $\mathrm{Sm}$ & S2 \\
\hline 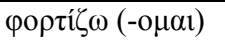 & 40,25 & p.p. tb & p.p. t & عمل soه \\
\hline$\varphi \rho o ́ v \eta \sigma ı s$ & 40,17 & טרמהלא & טרמהלה & $\begin{array}{l}\text { p.p. } \\
\text { rhassi }\end{array}$ \\
\hline 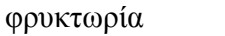 & 40,5 & ralial & ralin & Rhowimes \\
\hline 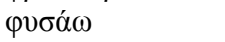 & 39,19 & נתר & sas & imshrer \\
\hline \multirow[t]{3}{*}{$\varphi \tilde{\omega} \varsigma$} & 39,1 & & Rims & rims \\
\hline & 40,1 & Ritss & rimes & rims \\
\hline & 40,3 & 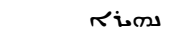 & rims & Rimos \\
\hline \multirow[t]{2}{*}{$\varphi \omega \tau \alpha \gamma \omega \gamma i ́ \alpha$} & 39,20 & dudiss & rimas didings & Rhorimsts \\
\hline & & rimos & & \\
\hline$\varphi \omega \tau i \zeta \omega(-o \mu \alpha \imath)$ & 39,14 & حداה & حמהו. & נחוi \\
\hline \multirow[t]{4}{*}{$\varphi \omega \tau \imath \sigma \mu o ́ \varsigma$} & 40,1 & " & Rimas & Rhouimsts \\
\hline & 40,5 & Rhouimess & Rhouimess & Rims \\
\hline & 40,24 & rimas & Rima & rhovimshos \\
\hline & 40,37 & Trimes & Rhouimess & Rhovimess \\
\hline 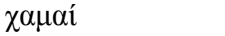 & 27,3 & 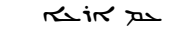 & & حل حiar \\
\hline$\chi \alpha \mu \varepsilon v v i ́ \alpha$ & 40,31 & 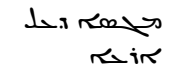 & 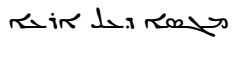 & 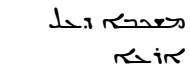 \\
\hline$\chi \alpha \rho \alpha \kappa \tau \eta \rho i ́ \zeta \omega$ & 39,12 & af. & نم pa. & نم pa. \\
\hline$\chi \alpha \rho \dot{\zeta} о \mu \alpha \imath$ & 40,12 & pa. نمi & pa. نم: & pa. غحم \\
\hline 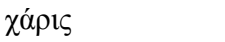 & 40,18 & rhanef & rhanel & rhawe \\
\hline \multirow[t]{2}{*}{$\chi \alpha ́ \rho ı \sigma \mu \alpha$} & 40,4 & rinor & Rino & عمحلم \\
\hline & 40,4 & Ror & Rion & عمحلم \\
\hline$\chi \varepsilon 1 \mu \alpha \dot{\alpha} \rho \rho о \circ \varsigma$ & 40,27 & ri & Rili & سلم \\
\hline 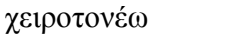 & 27,9 & מחד אחו.א & & af. \\
\hline \multirow[t]{2}{*}{ 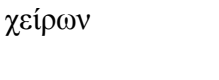 } & 27,7 & p.us & & حك, \\
\hline & 27,10 & p. sp & & Tres \\
\hline$\chi \varepsilon \dot{\varepsilon} \rho \sigma о \varsigma$ & 40,27 & ح.t. & טריאr & حسiٔم \\
\hline 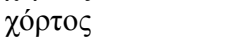 & 39,19 & טבגא & טרגא & حدكنم \\
\hline 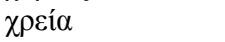 & 40,10 & Rhonaros & owaris & Rhoras \\
\hline \multirow[t]{2}{*}{$(\chi \rho \varepsilon i ́ \alpha \nu$ है $\chi \omega)$} & 39,15 & rotir & p. & p. \\
\hline & 39,15 & rotir & p. & p. معم \\
\hline 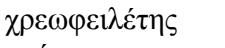 & 40,31 & טיב טaסרא & טיב טחסמא & טירא \\
\hline 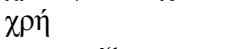 & 39,8 & p. $\bowtie$ o & p. s.n & p. In \\
\hline$\chi \rho \eta \mu \alpha \tau i \zeta \omega$ & 27,8 & etpa. Rnidir & & pa. \\
\hline$\chi \rho \eta \mu \alpha \tau \imath \sigma \tau \eta ́ s$ & 40,11 & مسلم & مسكم & rhi \\
\hline 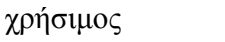 & 27,1 & Rurv & & p. \\
\hline$\chi \rho \tilde{\imath} \sigma \mu \alpha$ & $\begin{array}{l}40,15 \\
\text { (var.) }\end{array}$ & reves & ruess & Rhowess \\
\hline \multirow[t]{2}{*}{ 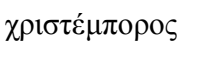 } & 40,11 & 蚁 & Ruess Ri d & is didir \\
\hline & & ruersix & & p. Rursu \\
\hline \multirow[t]{2}{*}{ 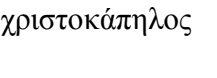 } & 40,11 & مaل xarsur & مa.لح x.surst & i pa. مa مues \\
\hline & 40,38 & $\begin{array}{c}\text { p. } \\
\text { arsas }\end{array}$ & sant & $\begin{array}{r}\text { p.p. } \\
\text { sat }\end{array}$ \\
\hline$\chi \omega ́ \rho \alpha$ & 40,35 & rhaox & Rhoo. & ritir \\
\hline$\chi \omega \rho \varepsilon ́ \omega$ & 27,7 & uir & & פחט \\
\hline$\chi \omega \rho \eta \tau 1 \kappa o ́ \varsigma$ & 40,39 & p. حهم & pa.p. فحل & كمحلنج \\
\hline \multirow[t]{2}{*}{$\psi \alpha \lambda \mu \omega \delta i ́ \alpha$} & 27,7 & Risal & & Rhesed \\
\hline & 40,46 & Risar & Risal & Rhesed \\
\hline$\psi \varepsilon v ́ \delta \omega(-o \mu \alpha \imath) \mathrm{p}$. & 40,37 & $\begin{array}{l}\text { sudir } \\
\text {, madela }\end{array}$ & יلحله & ירله \\
\hline$\omega \delta$ óv $\omega$ & 39,4 & & $\downarrow$ & va. vaد \\
\hline$\grave{\omega} \theta \dot{\varepsilon} \omega$ & 39,8 & ave & וحa & rus \\
\hline$(-o \mu \alpha \imath)$ & 27,8 & $\begin{array}{l}\text { asition } \\
\text { etpa. }\end{array}$ & & אוו.תר \\
\hline ஸ̋ $\sigma \varepsilon \rho \rho$ & 27,7 & אבתה & & אטמנת \\
\hline
\end{tabular}

${ }^{59}$ Le verbe vient de ברט.

${ }^{60}$ Une note marginale signale la variante Rhosims. 
Ce qui frappe d'abord, c'est la tendance en S2 à préférer une traduction étymologique. L'exemple le plus visible est celui des mots $\theta \varepsilon o \lambda o \gamma i ́ \alpha$ et $\theta \varepsilon o \lambda o ́ \gamma o s$. On constate que les versions anciennes et moyennes ont 1 'habitude ${ }^{61}$ de translittérer les mots grecs en revard et on alors respectivement, et que la version récente a recours à une traduction étymologique du mot

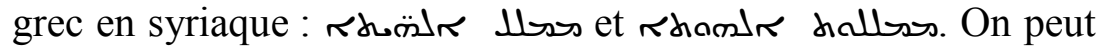

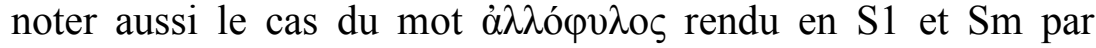

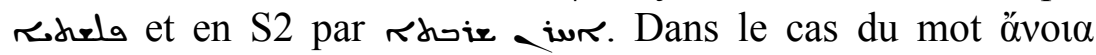
"déraison, sottise", il règne une certaine diversité dans les

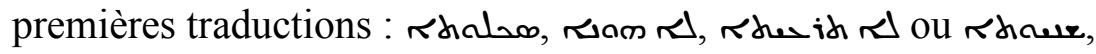
mais une uniformité en S2 qui traduit, en rendant le sens de l'alpha privatif, par ien ioieas "manque de réflexion». La

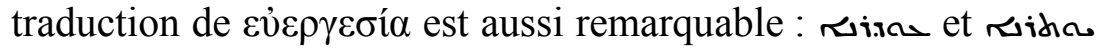
en S1 Sm, mais rhaح diainso "acte de bonté » la plupart du temps en S2 (mais une fois حris). Dans cette même ligne, les mots composés en grec (deux substantifs ou une préposition/préverbe + substantif/verbe) sont rendus par deux mots syriaques. Quelques exemples suffiront :

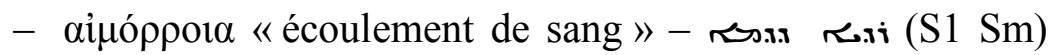
ou r.s dio.si (S2)

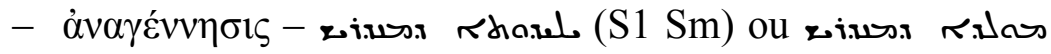
(S2)

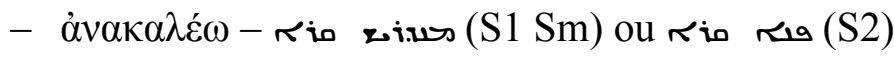

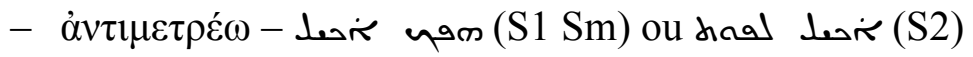

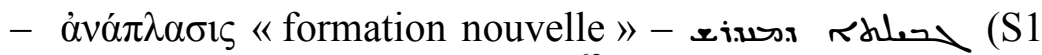
$\mathrm{Sm})$ ou riuss rhaten $(\mathrm{S} 2)^{62}$

On pourrait multiplier les exemples allant en ce sens. Mais il en est d'autres en sens contraire. Ainsi,

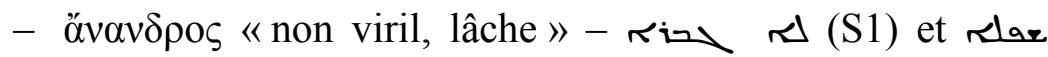
(S2)

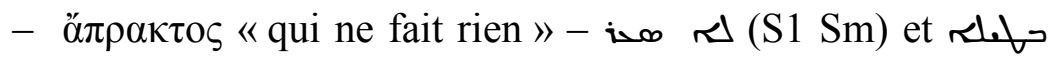
(S2)

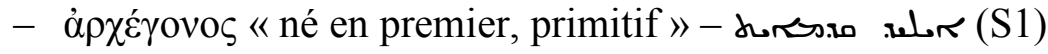
et م.דת (Sm S2).

${ }^{61}$ On a vu dans les notes qui accompagnent certains termes du tableau qu'il fallait bien se garder de systématiser. On parlera plutôt d'habitude ou de tendance à traduire tel terme grec de telle manière.

${ }^{62}$ On reviendra plus loin sur cette traduction. 
Il suffit de lire le tableau pour trouver de nombreux autres exemples. En voici deux autres extraits du Discours 1 :

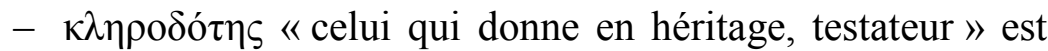
rendu selon l'étymologie en S1 par Rhatiz.x Ram, mais par un seul mot en S2 ritios.

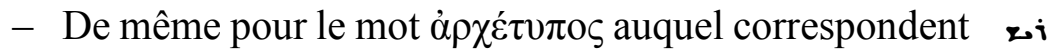
rugh (en S1) et ruad (en S2).

Lüdtke avait affirmé que S2 écartait les mots empruntés au grec pour les remplacer par des mots de formation sémitique. Quelques exemples viennent d'être vus. Voici d'autres cas relevés pour les discours $27,38-40^{63}$ :

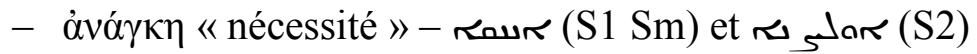

- $\dot{\alpha} \sigma \chi \eta \dot{\mu} \mu \nu$ 《difforme, inconvenant» $\mathrm{Sm},<\sigma \chi \tilde{\eta} \mu \alpha)$ et reoing (S2)

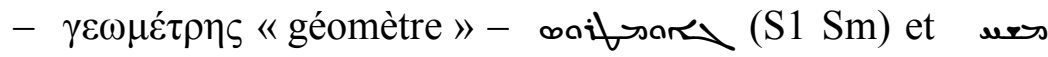
rir (S2, traduction étymologique)

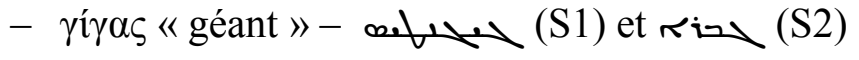

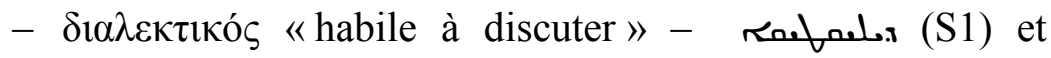
रimss (S2 « habile »)

- $\dot{\varepsilon} \kappa \delta 1 \alpha \mu \varepsilon ́ \tau \rho o v ~ « d a n s$ une direction diamétralement opposée »- a a (S1 Sm : simple translittération !) et Rhwors dowe (S2 "dans une mesure égale »)

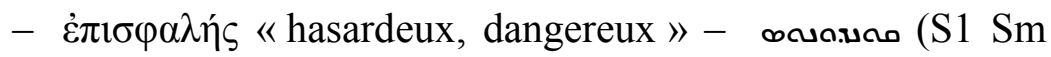

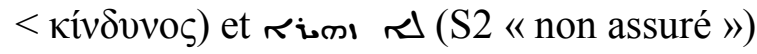

- $\theta \varepsilon \omega \rho \eta \tau \tilde{\omega} \varsigma$ «visible» - riarh $(\mathrm{S} 1 \mathrm{Sm},<\theta \varepsilon \omega \rho i ́ \alpha)$ et גorisudes (S2)

- $\lambda \alpha \mu \pi \eta \delta \omega ́ v ~ "$ clarté » - ruass (S1 Sm) et rus $(\mathrm{S} 2)$

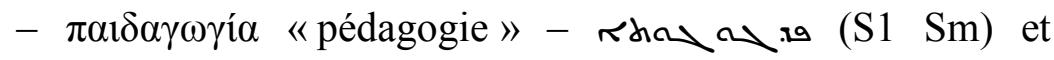
rharid (S2)

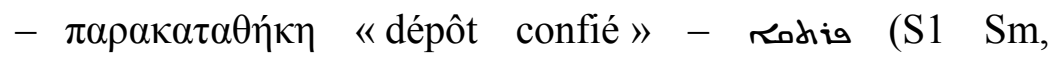

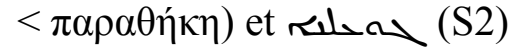

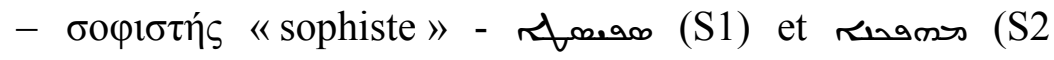
« pervers »)

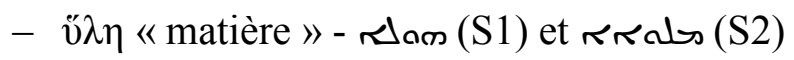

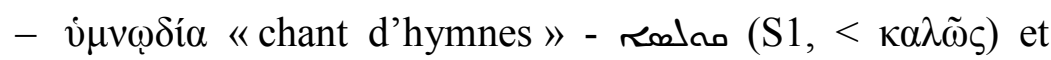
rusar (S2).

\footnotetext{
${ }^{63}$ On pourra en lire d'autres dans A. DE HALleuX, «L'homélie baptismale », p. 20-23.
} 
Mais il existe aussi bon nombre de cas où une traduction sémitique à l'origine (S1 Sm) a été remplacée par un décalque du grec en S2 :

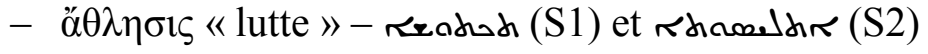

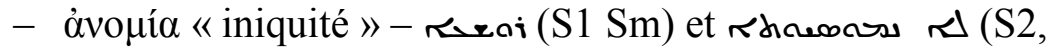
$<$ vó $\mu$ os, mais le mot grec avec ses dérivés a été profondément intégré dans la langue syriaque ${ }^{64}$ )

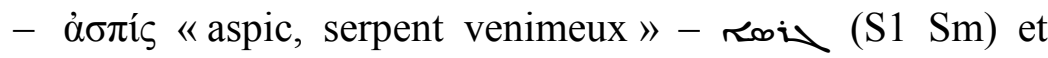
macor (S2)

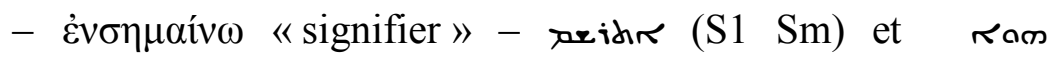

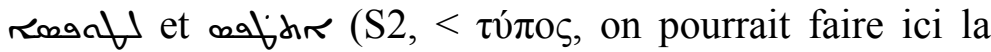
même remarque que pour le mot vó $\mu$ s)

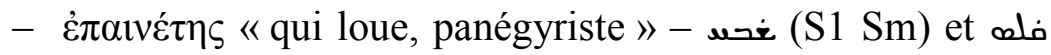
(S2, < $\kappa \alpha \lambda$ ós)

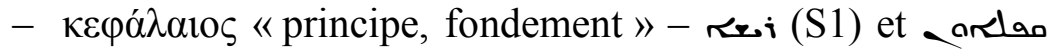
(Sm S2 [aussi גorass "en résumé »])

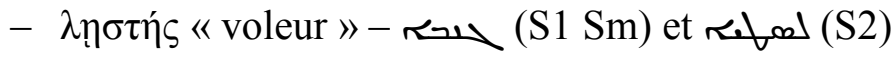

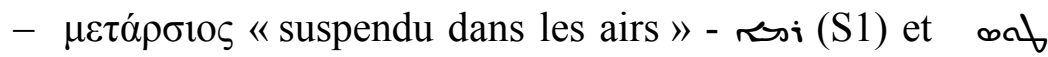

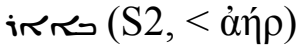

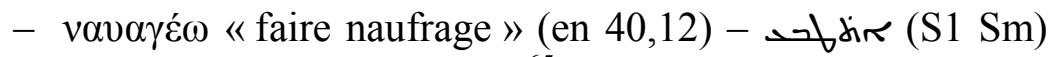
et $>$ osiin $\left(\mathrm{S} 2,<\operatorname{v\alpha v\alpha \gamma \varepsilon ́\omega )^{65}}\right.$

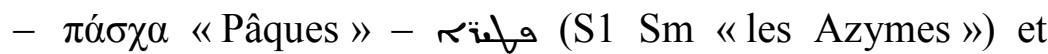
$\operatorname{sun}_{5} 9(\mathrm{~S} 2)$

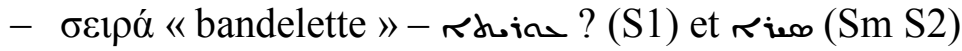

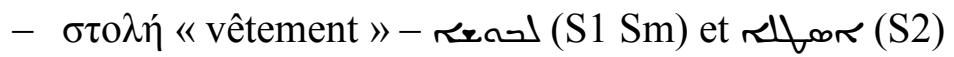

- $\sigma 0 \zeta \varepsilon v ́ \gamma v v \mu 1$ «mettre sous le même joug »- ح. (S1 Sm) et

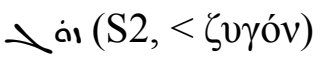

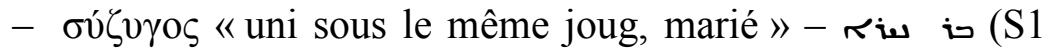

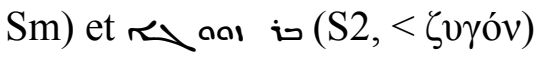

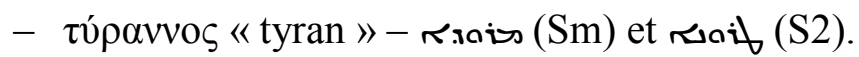

- L'exemple le plus frappant est celui de la traduction des trois termes théologiques parmi les plus prégnants que

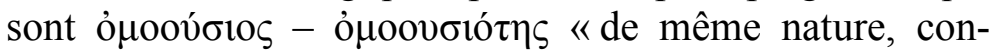

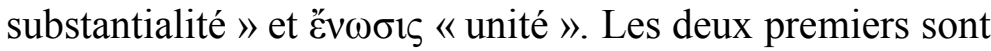

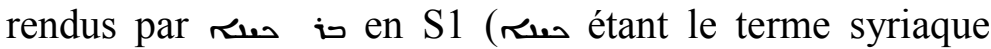

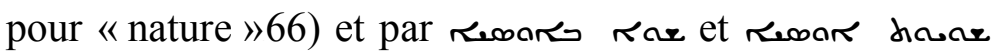

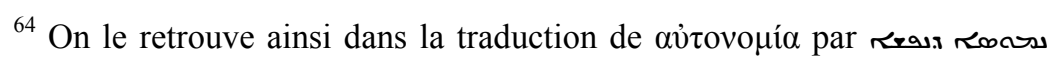
(S1 Sm) ou reras diocooss (S2).

${ }^{65}$ Mais en 40,11 on lit chez tous un dérivé du verbe grec : (S1 Sm) et $\rightarrow$ ostir (S2).

${ }^{66}$ Concile de Chalcédoine (451) : w2 (« une personne en deux natures »). Dans sa communication au colloque de Rome (voir n. 1) intitulée «Finding a Voice for Chrysostom : The Syriac Versions of a Greek 
en Sm S267. Le troisième par rhowsoul (S1) et rhorisu (Sm S2). Voici ce qu'en dit A. de Halleux : «Dans les trois cas, le vocabulaire ancien, qui remonte à coup sûr aux origines de $\mathrm{S} 1$, se trouve remarquablement préservé dans le témoin jacobite qu'est le ms. T, alors que tous les autres l'ont déjà normalisé en fonction de la terminologie monophysite du vie $\mathrm{s}$. Ces trois leçons de $\mathrm{T}$ autorisent donc à faire remonter la version syriaque des Discours audelà de cette époque ${ }^{68}$.

Le traducteur de S2, parfois déjà celui de Sm, corrige aussi par endroits une mauvaise traduction antérieure :

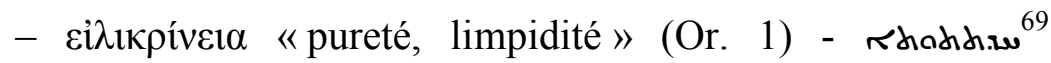
« nouveauté » $(\mathrm{S} 1)$ et rhiner $(\mathrm{S} 2)$,

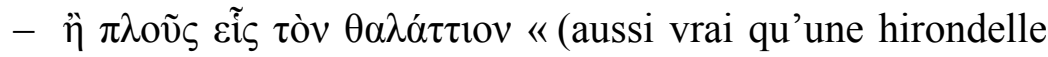
ne fait pas le printemps, ni une ligne le géomètre) ni une (unique) navigation l'homme de la mer » Or. 38,14] $\Delta a$

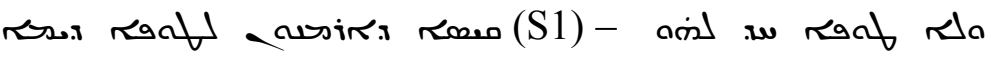
ress. (Sm S2). Sm et S2 (« et ce n'est pas un unique voyage en mer [qui fait] le marin ») ont corrigé S1 (« et ce

Preacher », J.W. Childers signale que le traducteur de Chrysostome utilise

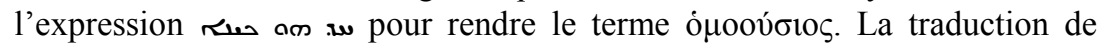
Chrysostome en syriaque a été réalisée aux environs de 500, cf. J.W. CHILDERS, « Chrysostom's Exegetical Homilies », p. 513.

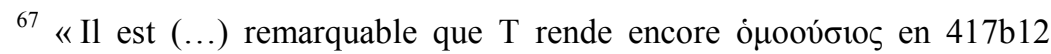
$[=40,41]$ et en $420 \mathrm{c} 1[=40,43]$, par le sémitisme viv cu l'ancienne version du symbole de Nicée-Constantinople alors qu'on trouve déjà chez UV, $\mathrm{W}$ et $\mathrm{X}[=\mathrm{fg} 40 \mathrm{a}]$ le grécisme ع 2 . La révision monophysite du symbole syriaque dans les premières années du $\mathrm{VI}^{\mathrm{e}} \mathrm{s}$. avait encore la forme rhadur, et reoara ne se généralisa dans la littérature théologique jacobite que dans le courant du même siècle. Dans ces conditions, la recension moyenne de la version syriaque des Discours grégoriens saurait difficilement être antérieure à la seconde moitié du $\mathrm{VI}^{\mathrm{e}}$ s. » A. DE HALlEuX, «L'homélie baptismale », p. 13-14. A. de Halleux considère en outre que le remplacement de حist vraisemblablement à mettre au compte de Philoxène de Mabbog. Ce dernier, dans ses premières œuvres, utilise encore

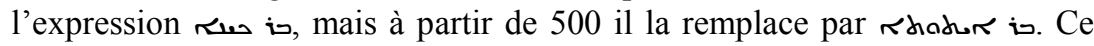

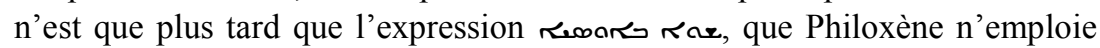
cependant pas, s'imposera comme terme technique christologique. Voir A. DE HALLEUX, « La Philoxénienne », p. 301-302.

${ }^{68}$ A. de Halleux, «L'homélie baptismale », p. 25-26. Il en trouve une confirmation dans le fait qu'en 40,10 le ms. T semble avoir conservé l'ancienne acception féminine de l'Esprit Saint: $\pi v \varepsilon \tilde{u} \mu \alpha \mu \varepsilon \dot{\varepsilon} v \dot{\varepsilon} \sigma \tau \iota v$, $\alpha \lambda \lambda \grave{\alpha}$ $\delta 1 \alpha \lambda \tilde{\text { ov }}$ őp « (oppose-lui l'Esprit Saint...) c'est un esprit, mais il fait fondre

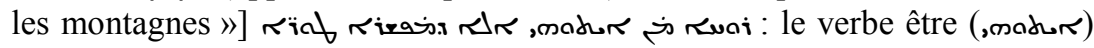
est bien pourvu du suffixe masculin, mais le participe (من nin : «cette faute d'accord trahissant peut-être un état du texte où le suffixe pronominal du verbe était, lui aussi, accordé au féminin ».

${ }^{69}$ Avec cette orthographe surchargée pour rhadiw. 
n'est pas le mât [qui fait] la navigation en mer »). La traduction de S1 provient vraisemblablement d'une mauvaise lecture de $\varepsilon 1 \varsigma \tau \mathrm{o}[v]$ en $1 \sigma \tau \mathrm{o}[\varsigma]$ « mât ».

Ou il l'améliore ou la rend plus précise :

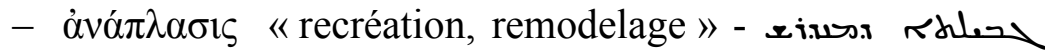
(S1 Sm) et בת (S2). Le traducteur de S2 fait une distinction entre $\pi \lambda \alpha ́ \sigma \mu \alpha$ " objet créé » et $\pi \lambda \alpha ́ \sigma 1 \varsigma$ « acte de créer » (dans le composé ỏvó $\pi \lambda \alpha \sigma ı \varsigma$ « recréation, remodelage ») : Paul d'Édesse rend en effet le premier par

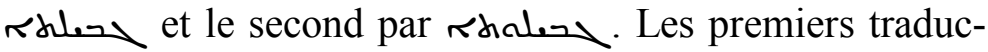
teurs n'avaient pas fait cette distinction: ils n'utilisaient que le premier terme.

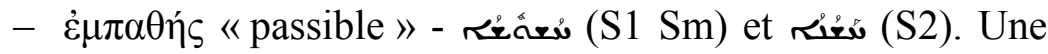
explication du changement pourrait être que le premier adjectif est devenu un terme technique pour désigner le passif grammatical. Il n'était donc plus très adapté dans un contexte théologique. On pourrait rendre le second adjectif syriaque par « pénible ».

- $\chi \alpha \rho \mu o ́ \sigma v v \alpha$ pluriel neutre de $\chi \alpha \rho \mu v ́ \sigma v v o \varsigma$ « qui est un sujet

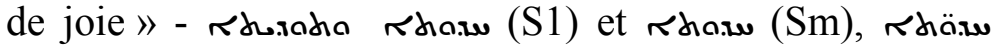
(S2). Le premier traducteur a utilisé deux substantifs («joie et remerciement») pour rendre l'adjectif neutre pluriel $^{70}$. La notion de gratitude n'apparaissant pas en grec, les deux traducteurs suivants n'ont retenu que le terme rhow; S2 le met même au pluriel pour mieux correspondre au grec.

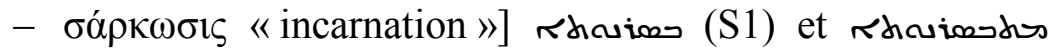
(Sm S2). Il faut citer ici le commentaire de A. de Halleux : «T ne témoigne plus ici de l'archaïsme rhosi traducteurs de Théodore de Mopsueste avaient peut-être créé en s'inspirant de l'expression riv de l'ancien symbole syriaque. Mais s'il emploie déjà le verbe iostir de la version du même symbole révisée au début du $\mathrm{VI}^{\mathrm{e}} \mathrm{s}$., il ne paraît pas encore connaître le terme technique rhoviosdos courant dans la christologie monophysite du $\mathrm{VI}^{\mathrm{e}} \mathrm{s}$. et qui apparaît dès la recension moyenne de $\mathrm{S} 1$ $[=\mathrm{Sm}] »^{71}$.

${ }^{70}$ A. DE HALlEUX, «L'homélie baptismale », p. 21, explique autrement cette leçon de S1 (représentée ici par le seul ms. T) en supposant que $\mathrm{T}$ a introduit ici une leçon marginale. A. de Halleux donne quelques exemples de ce phénomène, en particulier la double traduction de ả $\xi i ́ \alpha$ en 40,26 (voir tableau) par ric. Le ms. T, bien qu'il conserve pour l'essentiel le libellé de la première version, présente par endroits une forme déjà quelque peu révisée.

${ }^{71}$ A. DE HalleuX, «L'homélie baptismale », p. 24. Il renvoie à A. DE HalleuX, « La Philoxénienne », p. 303-305, 309-310. 
- Ě $\lambda \lambda \alpha \mu \psi 1 \varsigma$ "illumination》 - Rhouimes et Rhoims (S1), rhovimes, Rhaims et rhosimstos (Sm), Rhovimstss seul (S2). Cet exemple peut être rapproché du précédent : c'est un substantif formé sur le participe passif qui est préféré pour exprimer le fait d'être illuminé (ou incarné, cf. cidessus). On constate que Sm utilise déjà le terme préféré par S2.

- $\sigma \tilde{\omega} \mu \alpha$ 《corps » et ses dérivés] $\mathrm{Sm}$ ) et riva et ses dérivés (S2). C'est le corps du Christ qui est ici en jeu et c'est vraisemblablement sous l'influence de la liturgie (qui emploi $\kappa^{2}$ a pour désigner le corps du Christ: : placé par riva.

Il y a néanmoins beaucoup d'exemples où nous ne pouvons plus percevoir les raisons du changement. Sans doute s'agit-il simplement du remplacement d'un terme ressenti comme vieilli par un terme plus courant à l'époque de la traduction ${ }^{72}$.

Voici quelques exemples qu'on pourrait multiplier à l'envi :

- $\pi \rho o ́ \varsigma]$ dans le Discours 1 la version ancienne (S1) utilise toujours $\mathrm{S}_{5}(\mathrm{~S} 1)$ que la version récente $(\mathrm{S} 2)$ remplace systématiquement pas dal

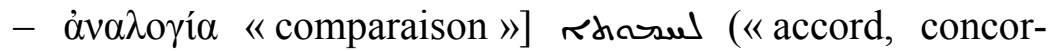
dance » S1 Sm) semble avoir été systématiquement remplacé en S2 par ssua ("comparaison») et par rhwars («mesure »)

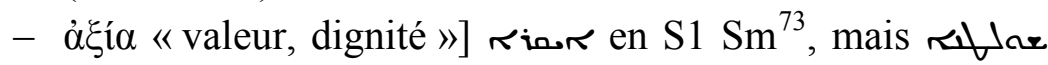
en $\mathrm{S} 2$

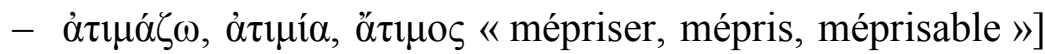
racine i $\tau_{5}$ en S1 Sm, mais racine $f_{a x}$ en S2

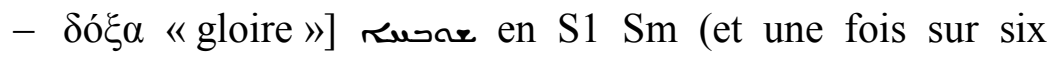
rhwoned) mais rhwored en S2 (et une fois sur six rusar)

\footnotetext{
${ }^{72}$ On observe un phénomène identique dans les vieilles versions latines de la Bible : au vocabulaire africain ancien des premières traductions (claritas, egenus, spado, etc.) a été substitué, avec le temps, un vocabulaire plus en usage en Italie par exemple (gloria, pauper, eunuchus, etc.). C'est un phénomène courant dans toutes les langues : qu'on compare les traductions françaises de la Bible du $18^{\mathrm{e}}$ et celles du $20^{\mathrm{e}}$ siècle. Un des apports de l'étude du vocabulaire des versions syriaques de Grégoire de Nazianze est de nous donner un éclairage sur l'histoire de la langue, en mettant en lumière les termes ressentis comme vieillis et ceux davantage en usage au $7^{\mathrm{e}} \mathrm{s}$. (à l'époque de Paul d'Édesse traducteur de S2).

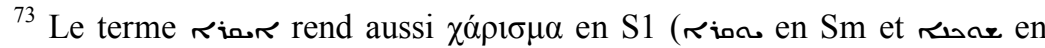
S2). 


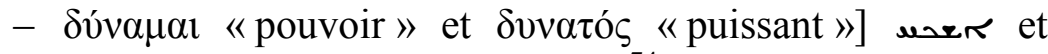
sers S1 Sm, mais $r_{5}$ en $2^{74}$

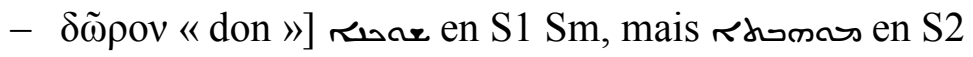

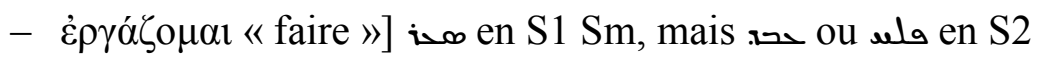

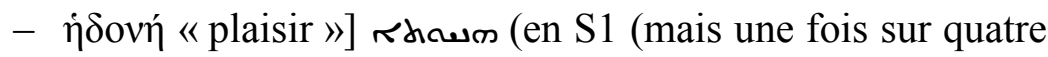
rhyi) Sm, mais rawien S2

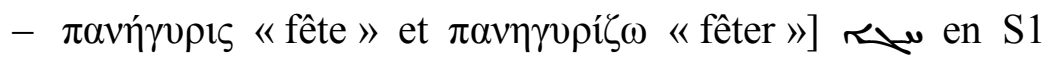
Sm, mais حa en S2

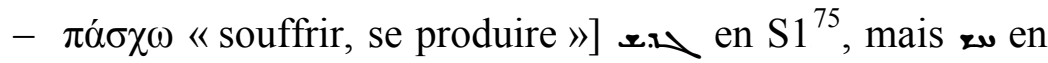
$\mathrm{S} 2$; les deux verbes apparaissent en Sm, mais avec une prédominance pour le second

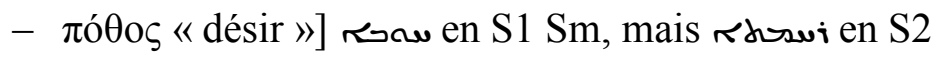

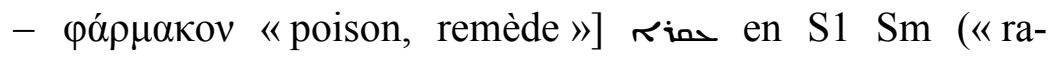
cine »), mais ssoe en S2.

Mais ce n'est pas seulement dans le vocabulaire que les différences apparaissent d'une version à l'autre, c'est aussi dans la manière de rendre la phrase entière du grec. Je ne note ici que quelques exemples. Les deux derniers montrent que la révision se poursuit par-delà $\mathrm{S} 1, \mathrm{Sm}$ et $\mathrm{S} 2$ : l'un illustre le travail de révision de Jacques d'Édesse, l'autre fait apparaître une tradition de leçons marginales qui constitue une étape supplémentaire dans le processus de révision.

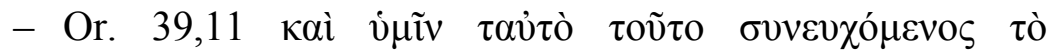

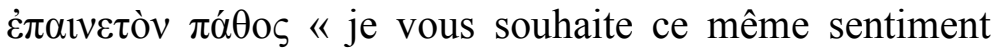

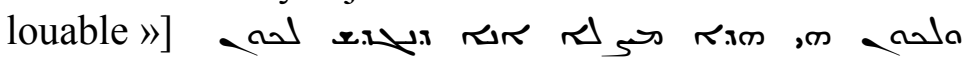

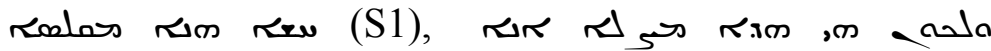

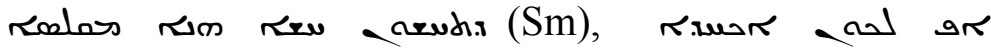
(S2). S1 et Sm ont ex-

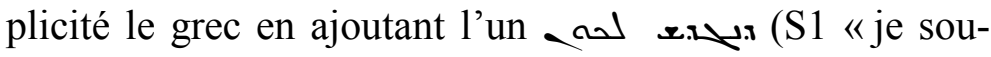
haite que vous advienne ce même sentiment louable ») l'autre arudis (Sm «je souhaite que vous éprouviez ce même sentiment louable »). S2 serre le grec de plus près : il supprime les ajouts de $\mathrm{S} 1$ et $\mathrm{Sm}$ et insère rendre le préverbe ovv- que les traducteurs antérieurs n'avaient pas exprimé. Il opère un autre changement qui mérite d'être souligné. Alors que les autres traducteurs

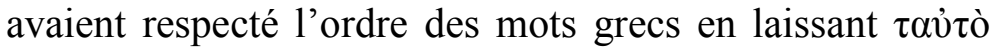

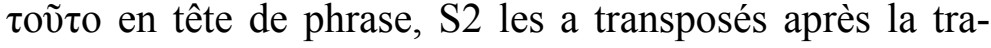

${ }^{74}$ La même alternance des termes se rencontre pour traduire le verbe i $\sigma \chi v \omega$ : (S1 Sm) et formes de (S2), ou le terme oĩos : et $r_{5} \rightarrow(\mathrm{S} 2)$.

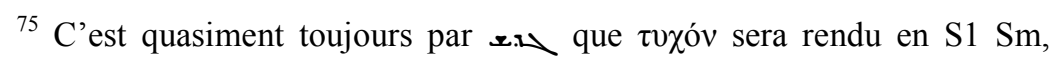
alors que S2 le rend par, atrer. 


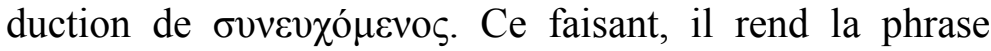
immédiatement plus compréhensible en syriaque.

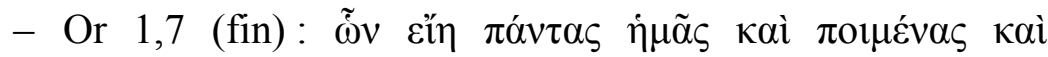

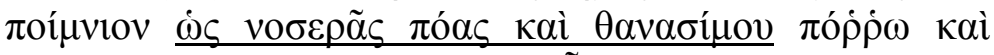

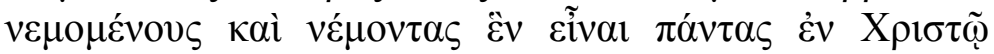
'I et troupeau, nous tenir éloignés comme d'une herbe qui apporte la maladie et la mort, quand nous conduisons le troupeau au pâturage ou que nous nous y laissons conduire, pour être tous un dans le Christ Jésus, etc. $\left.{ }^{76} \gg\right]$ קm

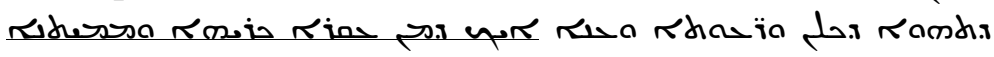
(S1),

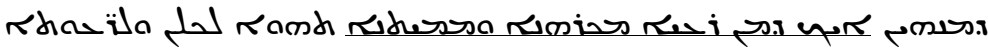

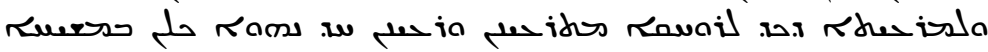
عمح (S2). Les cinq mots syriaques soulignés correspon-

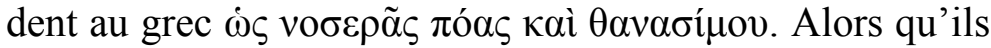
étaient conservés à leur place en S1, l'auteur de S2 les a déplacés juste après la traduction du pronom relatif grec. Un tel déplacement ne peut être le fait d'un traducteur qui entend donner une traduction miroir du grec; c'est bien davantage le fait d'un traducteur plus attentif au texte cible (« reader-oriented $\left.{ }^{77}\right)$ qu'au texte source.

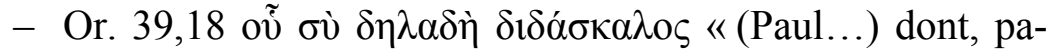

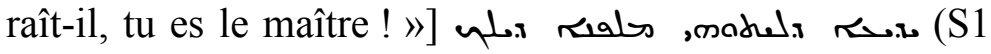

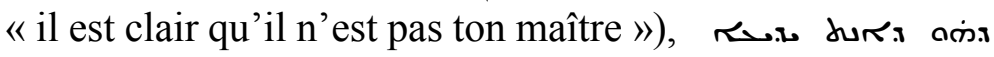
(Sm S2 « celui-là dont, c'est bien connu, tu es le maître ! »). Sm et S2, qui traduisent correctement le grec, ont corrigé S1 qui n'avait pas saisi l'ironie du passage.

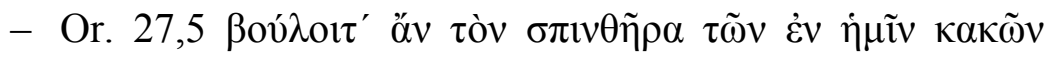
$\gamma \varepsilon v \varepsilon ́ \sigma \theta \alpha$ l $\varphi \lambda o ́ \gamma \alpha$ « lui qui voudrait que l'étincelle des maux qui sont en nous devienne une flamme ${ }^{78}$ ]

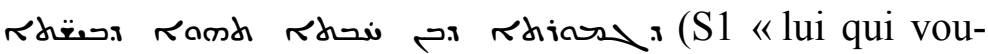
drait que l'étincelle qui brûle en nous devienne celle des

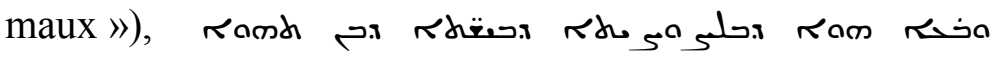
rhlin (S2). Rien qu'en respectant l'ordre des mots grecs, S2 a corrigé l'erreur du premier traducteur et présente une traduction correcte du grec. ${ }^{79}$

${ }^{76}$ Traduction de J. Bernardi.

77 Voir S. BROCK, «Aspects of Translation Technique in Antiquity », 1979 et ID., « Du grec en syriaque », 2005.

${ }^{78}$ Voici la traduction plus élégante de P. Gallay, p. 83 : « (eux) qui voudraient voir nos maux se transformer d'étincelle en flamme ».

${ }^{79}$ Les quelques exemples donnés ici pourraient être facilement multipliés. Il suffit pour cela de lire les notes critiques dans les volumes du syriaque déjà 


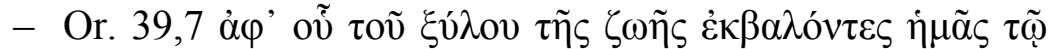

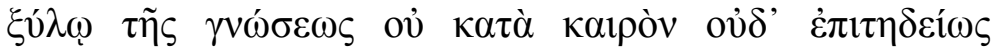
$\mu \varepsilon \tau \alpha \lambda \eta \varphi \theta \varepsilon i ́ \sigma \eta \varsigma$ « depuis qu'ils nous ont écartés loin de l'arbre de vie au moyen de l'arbre de la connaissance à laquelle nous avions pris part en-dehors du moment opportun et d'une manière inappropriée $\left.{ }^{80}\right]$ >

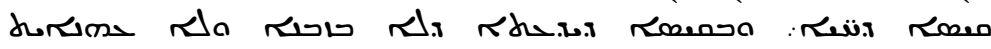

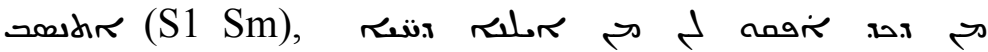

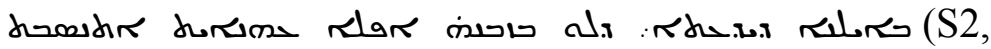

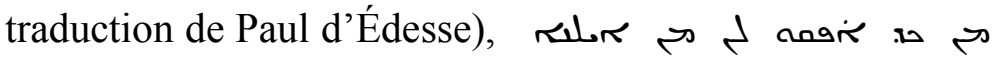

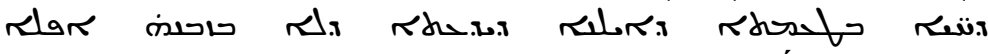
drooshr horac (S2, révision de Jacques d'Édesse). Les traducteurs syriaques ont éprouvé quelques difficultés à traduire cette phrase (où le participe $\mu \varepsilon \tau \alpha \lambda \eta \varphi \theta \varepsilon i ́ \sigma \eta \varsigma$ se rapporte à $\tau \tilde{\eta} \varsigma \gamma v \omega ́ \sigma \varepsilon \omega \varsigma$ : c'est la connaissance qui a été goûtée trop tôt). S1 et Sm ont ponctué après $\sim^{*}$ " r et ont ajouté une conjonction de coordination devant le mot qui suit. Ils ont mal interprété le grec : pour les premiers traducteurs, c'est à l'arbre de la connaissance qu'ils ont goûté, et non à la connaissance elle-même. Les premiers témoins de $\mathrm{S} 2$ (B, C, F, G, J, K, $\gamma, \delta, \kappa, \lambda$, traduction de Paul

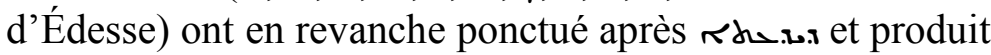
un texte correspondant au grec: «depuis qu'ils nous ont écartés de l'arbre de vie à cause de l'arbre de la connaissance à laquelle nous avions goûtée, etc. »; c'est à la connaissance qu'ils ont goûté trop tôt. Les témoins de la révision de Jacques d'Édesse (A, D, $\varepsilon, \eta)$ ont ajouté le mot rhos $(\gamma \varepsilon \tilde{\sigma} \sigma 1 \zeta)$ pour mieux rendre compte du féminin $\mu \varepsilon \tau \alpha \lambda \eta \varphi \theta \varepsilon i ́ \sigma \eta \varsigma$ : « depuis qu'ils nous ont écartés de l'arbre de vie, à cause du fait que nous avions goûté à l'arbre de la connaissance, (action) qui a été menée hors du moment voulu, etc. », mais du coup ils reviennent à l'interprétation des premiers traducteurs. Révision n'est donc pas toujours synonyme d'amélioration.

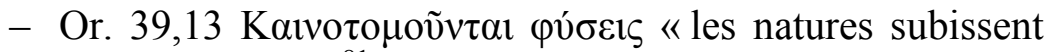
une innovation ${ }^{81}$ ]

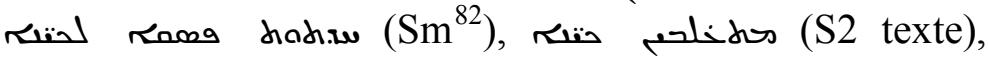

édités où les divergences entre les versions syriaques et le grec sont toujours expliquées.

${ }^{80}$ P. Gallay, p. 161 : «(Qu'ils se livrent donc à ces bouffonneries...ces démons...qui divisent les hommes...) depuis qu'ils nous ont écartés de l'arbre de vie, parce que nous avions touché à l'arbre de la connaissance hors du moment voulu et sans égard à ce qui convenait. »

${ }^{81}$ Traduction de P. Gallay.

82 «Intervient une innovation pour les natures » (litt. « entre une nouveauté de coupure pour les natures »). 


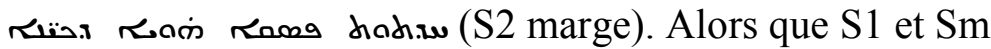
avaient donné une traduction étymologique du verbe

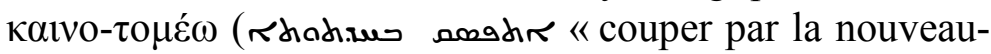
té »), S2 a recours au verbe passé, être opprimé » qui s'éloigne du grec $^{83}$. Pourquoi Jacques d'Édesse n'a-t-il pas revu cette traduction? Quoi qu'il en soit, le texte de S2 a été ressenti comme insatisfaisant par les premiers copistes qui ont tous proposé en marge une traduction plus fidèle au grec.

\section{Le modèle grec}

Les éditeurs du texte grec des Discours de Grégoire de Nazianze, à la suite de l'étude fondamentale de A. Sinko et de celle de V. Somers ${ }^{84}$, ont dégagé dans la tradition manuscrite deux familles principales ( $m$ et $n$ ) auxquelles vient s'ajouter une famille $\mathrm{x}$ regroupant les autres manuscrits qui ne sont ni $\mathrm{m}$ ni $\mathrm{n}$. La famille $\mathrm{m}$ comprend les manuscrits suivants (dans l'ordre signalé par les éditeurs) :

- S Mosquensis Synodalis 17, Vladimir 139 du $9^{\mathrm{e}} \mathrm{s}$.

- P Patmiacus 33, daté de 941

- Pd Parisinus Graecus 515 du $9^{\mathrm{e}}$ s.

- C Parisinus Coislianus 51, du $10^{\mathrm{e}}-11^{\mathrm{e}} \mathrm{s}$.

- R Vaticanus Graecus 2061a du 10 e s.

- O Vaticanus Ottobonianus gr. $396 \mathrm{du} 10^{\mathrm{e}} \mathrm{s}$.

- Ve Vaticanus graecus $1805 \mathrm{du} 10^{\mathrm{e}} \mathrm{s}$.

- Vb Vaticanus graecus 462 du $9^{\mathrm{e}} \mathrm{s}$.

- Vp Vaticanus Palatinus gr. 75 du $10^{\mathrm{e}} \mathrm{s}$.

- D Marcianus Graecus 70 du $10^{\mathrm{e}} \mathrm{s}$.

Pour la famille n, les éditeurs ont utilisé les sept manuscrits suivants :

- A Ambrosianus E 50 inf. gr. 1014 du $9^{\mathrm{e}} \mathrm{s}$.

- B Parisinus Graecus 510 du $9^{\mathrm{e}}$ s.

- W Mosquensis Synodalis 64, Vladimir 142, du $9^{\mathrm{e}} \mathrm{s}$.

- Q Patmiacus $44 \mathrm{du} 10^{\mathrm{e}} \mathrm{s}$.

- T Mosquensis Synodalis 53, Vladimir 147, du $10^{\mathrm{e}} \mathrm{s}$.

- V Vindobonensis theol. gr. 126 du début du $11^{\mathrm{e}} \mathrm{s}$.

- Z Vaticanus Graecus $1249 \mathrm{du} 10^{\mathrm{e}} \mathrm{s}$.

83 PAYNE-SMith, Thesaurus, col. 2893-2894 ne donne aucun autre exemple de cette traduction du verbe grec.

${ }^{84}$ A. SINKO, De traditione, 1917 ; V. SOMERS, Histoire des collections complètes, 1997. 
Tous ces manuscrits ne sont pas complets, et certains présentent même de grandes lacunes. Les deux familles s'opposent tout au long des Discours 38-40 et la famille m semble présenter un texte légèrement plus long que celui de la famille $n$.

Cl. Moreschini, l'éditeur des Discours 38-40, signale les variantes les plus marquantes où les deux familles s'opposent ${ }^{85}$. Comparons ces variantes avec le témoignage du syriaque ( $\mathrm{S} 1$ et Sm ne sont pas toujours attestés).

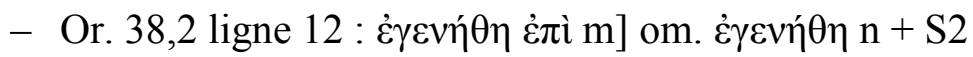

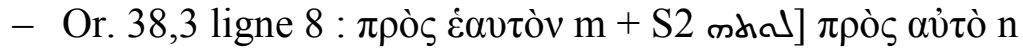

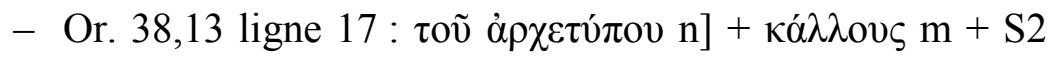
Rians:

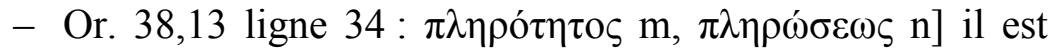
impossible de trancher car S2 traduit par shads qui peut correspondre aux deux termes grecs

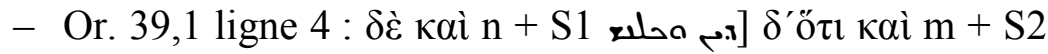
דיה גיזפ حلי

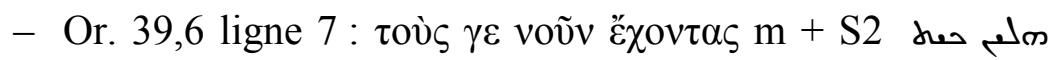

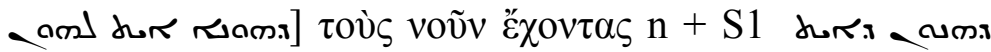
wam and

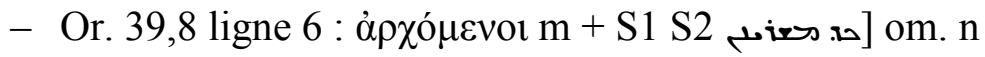

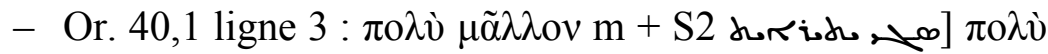
$\pi \lambda \varepsilon$ ĩov $\mathrm{n}+\mathrm{S} 1 \mathrm{Sm} \sim \mathrm{i} \downarrow,-\infty$, mais le doute est permis

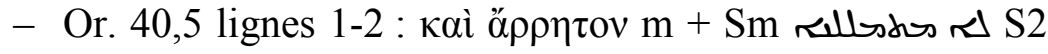

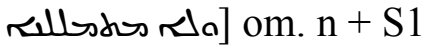

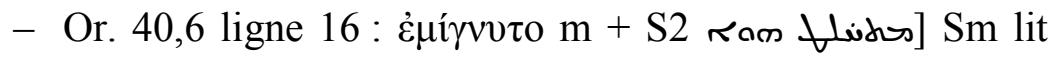
$\forall$ Vudire, à savoir un parfait qui ne correspond pas au pré-

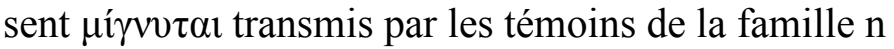

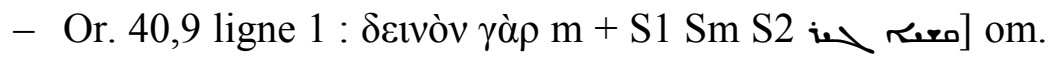
yà $\mathrm{n}$

- Or. 40,23 lignes 28ss : ajout des mots suivants (absents en

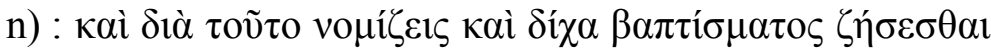

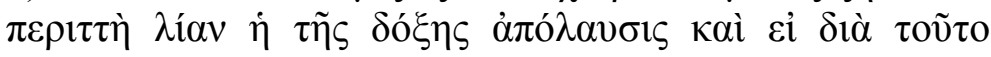

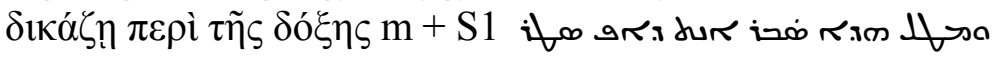

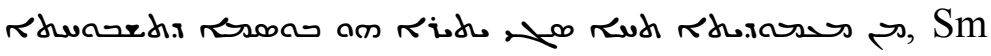

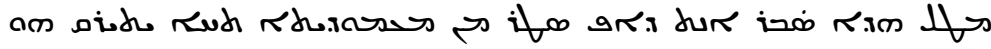

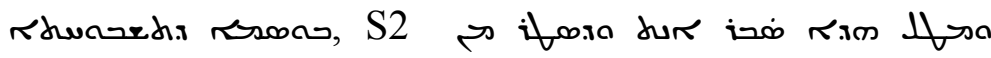
rhwoned dal naod dur rew rhososs. La tradition syriaque entière contient cette addition, mais elle n'y apparaît pas sous la même forme. En $\mathrm{S} 1$ et Sm les mots kaì عì

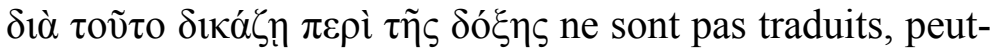
être par omission par passage du même au même en sy-

\footnotetext{
${ }^{85}$ Cl. Moreschini - P. Gallay, Grégoire de Nazianze. Discours 38-41, p. $90-93$.
} 


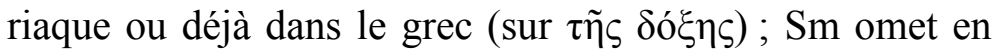

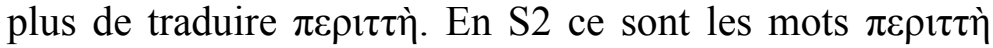

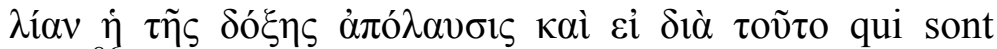
omis $^{86}$.

- Après Or. 38,6 ligne 9 apparaît dans quelques témoins de la famille m (S, R, O, Vp, Ve et le Laur. VII,8 du $11^{\mathrm{e}} \mathrm{s}$.) un long additamentum de plus de 10 lignes ${ }^{87}$, et on le retrouve en syriaque dans S2. L'additamentum a été considéré comme authentiquement grégorien par Sinko, mais comme inauthentique par les Mauristes (il est donc absent de la $P G$ ) et par Cl. Moreschini qui y voit une interpolation tardive. Toutefois A. Van Roey a découvert une attestation de ce passage dans la tradition syriaque indirecte chez Pierre de Callinique ( $6^{\mathrm{e}}$ s. $)^{88}$. L'interpolation est donc ancienne. Malheureusement le témoignage du syriaque ne permet pas d'aller plus loin, car seule la version S2 (datée de 623-624) est attestée pour ce passage, et elle est bien postérieure au témoignage de Pierre de Callinique.

Que conclure de cette analyse des variantes grecques comparées aux textes syriaques? Si l'on met à part les quelques cas où il est impossible de juger, il est clair que la version S2 concorde majoritairement avec les témoins de la famille m (voir en particulier l'ajout en 40,23 lignes 28-31 et l'additamentum). Cet accord avec la famille $\mathrm{m}$ apparaît déjà en $\mathrm{S} 1$ et $\mathrm{Sm}$, encore que ces derniers par endroits manifestent des accords remarquables avec certaines leçons de la famille $n$.

\section{Conclusions}

Que ce soit dans 1'analyse du vocabulaire ou dans celle des manières de rendre la phrase grecque, on a pu constater qu'était à l'œuvre un processus continuel de révision et d'adaptation. La première traduction syriaque, à situer au $5^{\mathrm{e}}$ (avec A. de Halleux, et non au $6^{\mathrm{e}} \mathrm{s}$. avec $\mathrm{S}$. Brock), a fait l'objet d'une première révision qui apparaît, après 550, dans Sm (pour certains Discours seulement); elle est encore relativement légère, mais certaines de ses modifications seront reprises par la suite. En effet, à leur tour, S1 et Sm ont été revus en 623/624 par Paul d'Édesse,

${ }^{86}$ Les indications données par la $P G$, par Moreschini et par de Halleux, «L'homélie baptismale », p. 31, ne correspondent pas exactement à ce qu'on lit dans le syriaque.

${ }^{87}$ Il est trop long pour être noté ici, mais on peut le lire dans A. SINKO, De traditione, p. 168 et dans CL. MORESCHINI - P. GALlAY, Grégoire de Nazianze. Discours 38-41, p. 92.

${ }^{88}$ A. VAN ROEY, «Une attestation syriaque », 1996. J'ai repris ce texte dans J.-Cl. HAELEWYCK, Sancti Gregorii Nazianzeni Opera. Versio Syriaca. III. Orationes XXVII, XXXVIII, XXXIX, 2005, p. viii. Le texte de S2 ne correspond pas tout-à-fait à celui de Pierre de Callinique. 
l'auteur de S2, pour mieux adapter le texte syriaque au texte grec (correspondant davantage avec les témoins grecs de la famille $\mathrm{m}$ ), mais cette révision n'a pas toujours été menée de façon systématique ; on l'a vu en particulier dans l'élimination des grécismes. Il est apparu aussi que certains témoins manuscrits avaient conservé des traces d'une révision ultérieure, attribuée à Jacques d'Édesse ( $7^{\mathrm{e}} \mathrm{s}$.). Enfin, par endroits, certaines leçons marginales poursuivent le processus de révision en proposant des leçons alternatives plus proches du grec ou simplement des éclaircissements sur la manière de le comprendre. 


\section{Annexe: Les manuscrits syriaques des Discours de Gré- goire de Nazianze $e^{89}$}

La version ancienne (S1)

$\mathrm{T}$ : Londres, British Library, Add. $17146\left(1^{\text {ère }}\right.$ moitié du $\left.8^{\mathrm{e}} \mathrm{s}.\right)$

La version moyenne ou intermédiaire (Sm)

UV : Londres, British Library, Add. $14546+$ Add. 18813 ( $7^{\mathrm{e}}$ s.)

W : Londres, British Library, Add. $18815\left(9^{\mathrm{e}}-10^{\mathrm{e}} \mathrm{s}\right.$.)

Fg40a : Londres, British Library, Add. $14538\left(10^{\mathrm{e}} \mathrm{s}.\right)$

Fg40c : Londres, British Library, Add. $17191\left(9^{\mathrm{e}}\right.$ ou $10^{\mathrm{e}}$ s.)

La version récente (S2)

$\underline{\text { Les manuscrits du premier tome }}$

A : Londres, British Library, Or. 8731 (daté de 834)

B : Londres, British Library, Add. 14548 (daté de 790)

C : Londres, British Library, Add. 12153 (daté de 844/845)

D : Londres, British Library, Or. 8730 (daté de 876/877)

$\mathrm{E}$ : Mardin, Bibliothèque de l'archevêché syrien orthodoxe (incomplet)

F : British Library, Add. 14547 ( $9^{\mathrm{e}}$ s.)

G : Londres, British Library, Richianus $7187\left(9^{\mathrm{e}} \mathrm{s}.\right)$

$\mathrm{H}$ : Leningrad (St. Pétersbourg), Bibliothèque Publique, nouvelle série syr. $12\left(8^{\mathrm{e}} \mathrm{s}\right.$.)

J : Damas, syr. 3/19 ( $8^{\mathrm{e}}$ s.)

$\mathrm{K}$ : Paris, Bibliothèque nationale de France, syr. $376\left(9^{\mathrm{e}} \mathrm{s}.\right)$

L : British Library, Add. 10967 ( $9^{\mathrm{e}}$ s.)

( $\mathrm{M}, \mathrm{N}, \mathrm{O}:$ non attribués)

P : Londres, British Library, Add. $14549\left(8^{\mathrm{e}}-9^{\mathrm{e}}\right.$ s. $)$

Q : Harvard, Houghton Library, syr. $46\left(10^{\mathrm{e}} \mathrm{s}.\right)$

R1 : Paris, Bibliothèque nationale de France, syr. $378\left(8^{\mathrm{e}}-9^{\mathrm{e}} \mathrm{s}.\right)$

R2 : Birmingham, Selly Oak, Mingana sur. $662\left(8^{\mathrm{e}}-9^{\mathrm{e}}\right.$ s. $)$

(S non attribué)

${ }^{89}$ Tous les manuscrits syriaques contenant les Discours de Grégoire ont été décrits dans A. VAN ROEY - H. MOORS, «Les Discours » et dans A. SCHMIDT - M. QUASCHNING-KIRSCH, « Die syrischen Handschriften ». 


\section{Les manuscrits du second tome}

P : Londres, British Library, Add. $14549\left(8^{\mathrm{e}}-9^{\mathrm{e}}\right.$ s. $)$

Fg40b : Londres, British Library, Add. 14725, fol. 1-95 (10 $\mathrm{e}$ s.)

Les homéliaires

$\alpha$ : Città del Vaticano, Vat. syr. $368\left(8^{\mathrm{e}} \mathrm{s}.\right)$

$\beta$ : Città del Vaticano, Vat. syr. $253\left(8^{\mathrm{e}} \mathrm{s}\right.$.)

$\gamma$ : Berlin, Staatsbibliothek, Sachau $220\left(8^{\mathrm{e}}-9^{\mathrm{e}}\right.$ s. $)$

$\delta$ : Città del Vaticano, Vat. syr. $369\left(9^{\mathrm{e}} \mathrm{s}\right.$. $)$

$\varepsilon$ : Londres, British Library, Add. $14516\left(9^{\mathrm{e}} \mathrm{s}\right.$.)

$\zeta$ : Londres, British Library, Add. 14601 ( $9^{\mathrm{e}} \mathrm{s}$.)

$\eta$ : Londres, British Library, Add. 14515 (daté de 893)

$\theta$ : Londres, British Library, Add. $14725\left(10^{\mathrm{e}} \mathrm{s}\right.$.)

1 : Damas, Patriarcat syrien orthodoxe, syr. 12/20 (daté de 1000)

$\kappa$ : Damas, Patriarcat syrien orthodoxe, syr. 12/19 (11 $\mathrm{e}$ s.)

$\lambda$ : Londres, British Library, Add. 12165 (daté de 1015)

$\mu$ : Birmingham, Selly Oak, Mingana syr. 545 (daté de 1929) 


\section{BIBLIOGRAPHIE}

Assemani J.S., Bibliotheca Orientalis Clementino-Vaticana, 3 vol., Rome, 1719-1728 (réimpr. Hildesheim - New York, 1975).

BAUMSTARCK A., Geschichte der syrischen Literatur mit Ausschluss der christlich-palästinensichen Texte, Bonn, 1922.

BERNARDI J, Grégoire de Nazianze. Discours 1-3. Introduction, texte critique, traduction et notes (Sources Chrétiennes 247), Paris, 1978.

BROCK S., "Aspects of Translation Technique in Antiquity », Greek, Roman and Byzantine Studies 20, 1979, p. 69-87, repris dans S. Brock, Syriac Perspectives on Late Antiquity (Collected Studies Series), Londres, 1984.

BROCK S., « Du grec en syriaque : l'art de la traduction chez les Syriaques », dans Les Syriaques transmetteurs de civilisations. L'expérience $d u$ Bilad-el-Sham à l'époque omeyyade (Patrimoine Syriaque. Actes du Colloque IX), Antelias - Paris, 2005, p. 11-34.

Brock S., "The Armenian and Syriac Versions of the PsNonnos Mythological Scholia », Le Muséon 79, 1966, p. 401428.

Brock S., The Syriac Version of the Pseudo-Nonnos Mythological Scholia, Cambridge, 1971 (cité S. Brock, Pseudo-Nonnos).

BROCK S., «Zur Überlieferungsgeschichte der Nonnos zugeschriebenen Scholien im Syrischen ", dans XVII. Deutscher Orientalistentag...1968 in Würzburg (= Zeitschrift der Deutschen Morgenländischen Gesellschaft t. Suppl. 1/2), Wiesbaden, 1969, p. 458-462.

BUdILOVICH A., XIII slov Grigoriya Bogoslova $v$ drevneslavyanskom perevode po rukopisi Imperatorskoy Publichnoy Biblioteki XI veka. Kritiko-paleografichesky trud, St. Pétersbourg, 1875.

ChILders J.W., "Chrysostom's Exegetical Homilies on the New Testament in Syriac Translation », dans E.A. LivingstonE (éd.), Studia Patristica Vol. XXXIII, Leuven, 1997, p. 509-516.

Coulie B. - Metreveli H. - Bezarachvili K. - Kourtsikidze T. - MelikichVili N. - OtKhMezuri Th. - Raphava M., Sancti Gregorii Nazianzeni Opera. Versio Iberica. IV. Oratio XLIII (Corpus Christianorum. Series Graeca 52, Corpus Nazianzenum 17), Turnhout - Leuven, 2004.

Coulie B. - Metreveli H. - Bezarachvili K. - Kourtsikidze T. - MelikichVili N. - RaphaVA M., Sancti Gregorii Nazianzeni Opera. Versio Iberica. V. Orationes XXXIX, XL (Corpus Christianorum. Series Graeca 58, Corpus Nazianzenum 20), Turnhout - Leuven, 2007. 
Coulie B. - SiRInIAN A., Sancti Gregorii Nazianzeni Opera. Versio Armeniaca. III. Orationes XXI, VII, VIII (Corpus Christianorum. Series Graeca 38, Corpus Nazianzenum 7), Turnhout - Leuven, 1999.

Coulie B., Sancti Gregorii Nazianzeni Opera. Versio Armeniaca. I. Orationes II, XII, IX (Corpus Christianorum. Series Graeca 28, Corpus Nazianzenum 3), Turnhout - Leuven, 1994.

CoulIE B., Sancti Gregorii Nazianzeni Opera. Versio Iberica. VI. Orationes XI, XXI, XLII (Corpus Christianorum. Series Graeca 78, Corpus Nazianzenum 26), Turnhout - Leuven, 2013.

DE HalleuX A., «L'homélie baptismale de Grégoire de Nazianze. La version syriaque et son apport au texte grec », Le Muséon 95, 1982), p. 5-40 (cité A. DE HALlEuX, «L'homélie baptismale »).

DE HalleuX A., «La Philoxénienne du symbole», dans F. GrafFin - A. Guillaumont (éd.), Symposium Syriacum 1976 célébré du 13 au 17 septembre 1976 au Centre Culturel 'Les Fontaines' de Chantilly (France) (Orientalia Christiana Analecta 205), Rome, 1978, p. 295-315 (cité A. DE HALlEuX, « La Philoxénienne »).

DE HalleuX A., « La version syriaque des Discours de Grégoire de Nazianze », dans J. MossAy (éd.), II Symposium Nazianzenum, 1983, p. 75-111 (cité A. DE HALLEUX, «La version syriaque $\gg)$.

DE HALleuX A., « Les commentaires syriaques des discours de Grégoire de Nazianze. Un premier sondage », Le Muséon 98, 1985, p. 103-147 (cité A. DE HALlEUX, «Les commentaires syriaques $»)$.

DE HalleuX A., «Rabban Benjamin d'Édesse et la date du ms. B.L., Or. $8731 »$, dans H.J.W. DRIJVERS - R. LAVENANT - MoLENBERG C. - REININK G.J. (éds), IV Symposium Syriacum 1984. Literary Genres in Syriac Literature (Orientalia Christiana Analecta 229), Rome, 1987, p. 445-451 (cité A. DE HALleuX, «Rabban Benjamin »).

Engelbrecht A., Tyrannii Rufini Orationum Gregorii Nazianzeni novem interpretatio (Corpus Scriptorum Ecclesiasticorum Latinorum 46), Vienne, 1910.

Gallay P. avec la collaboration de M. JOURJON, Grégoire de Nazianze. Discours 27-31 (Discours théologiques). Introduction, texte critique, traduction et notes (Sources chrétiennes 250), Paris, 1978 (cité Traduction de P. Gallay).

Grand'Henry J., Sancti Gregorii Nazianzeni Opera. Versio Arabica antiqua. I. Oratio XXI (arab. 20) (Corpus Christianorum. Series Graeca 34, Corpus Nazianzenum 4), Turnhout Leuven, 1996. 
GRAND'Henry J., Sancti Gregorii Nazianzeni Opera. Versio Arabica antiqua. III. Oratio XL (arab. 4) (Corpus Christianorum. Series Graeca 57, Corpus Nazianzenum 19), Turnhout Leuven, 2005.

Grand'Henry J., Sancti Gregorii Nazianzeni Opera. Versio Arabica antiqua. II. Orationes XI, XLI (arab. 8. 12) (Corpus Christianorum. Series Graeca 85, Corpus Nazianzenum 27), Turnhout - Leuven, 2013.

GRASSELLINI M., « La tradizione slava antica delle omelie di S. Gregorio di Nazianzino », Europa Orientalis 11, 1992, p. 181195.

HaelewyCK J.-CL., Sancti Gregorii Nazianzeni Opera. Versio Syriaca. I. Oratio XL (Corpus Christianorum. Series Graeca 49, Corpus Nazianzenum 14), Turnhout - Leuven, 2001.

Haelewyck J.-Cl., Sancti Gregorii Nazianzeni Opera. Versio Syriaca. III. Orationes XXVII, XXXVIII, XXXIX (Corpus Christianorum. Series Graeca 53, Corpus Nazianzenum 18), Turnhout - Leuven, 2005.

Haelewyck J.-Cl., Sancti Gregorii Nazianzeni Opera. Versio Syriaca. IV. Orationes XXVIII, XXIX, XXX, XXXI (Corpus Christianorum. Series Graeca 65, Corpus Nazianzenum 23), Turnhout - Leuven, 2007.

Haelewyck J.-CL., Sancti Gregorii Nazianzeni Opera. Versio Syriaca. V. Orationes I, II, III (Corpus Christianorum. Series Graeca 77, Corpus Nazianzenum 25), Turnhout - Leuven, 2011.

ISEBAERT-CAUUET I., «Les Pères grecs dans les commentaires syriaques ", dans A. SCHMIDT - D. Gonnet (éd.), Les Pères grecs dans la tradition syriaque (Études syriaques 4), Paris, 2007, p. 77-88.

LAFONTAine G. - Metreveli H., «Les versions copte, arménienne et géorgienne de Saint Grégoire le Théologien. Etat des recherches ", dans J. MossAY (éd.), II. Symposium Nazianzenum, Paderborn - München - Wien - Zürich, 1983, p. 63-73.

LAFONTAINE G., " La version copte sahidique du Discours 'Sur la Pâque' de Grégoire de Nazianze », Le Muséon 93, 1980, p. $37-52$.

LAFONTAINE G., « La version copte bohaïrique du Discours 'Sur l'amour des pauvres' de Grégoire de Nazianze, Le Muséon 93, 1980, p. 199-236.

LAFOnTAINE G., « La version copte des Discours de Grégoire de Nazianze », Le Muséon 94, 1981, p. 37-45.

LÜDTKE W., «Zur Überlieferung der Reden Gregors von Nazianz », Oriens Christianus 13, 1913, p. 263-276.

MACÉ C. - DUBUISSON M., «L'apport des traductions anciennes à l'histoire du texte de Grégoire de Nazianze. Application au 
Discours $2 »$, Orientalia Christiana Periodica 69, 2003, p. 287340.

Metreveli H. - BezarachVili K. - Dolakidze M. - KourtsiKiDZE T. - MatchaVARiani M. - MelikishViLi N. - RAPHAVA M. - Chanidze M., Sancti Gregorii Nazianzeni Opera. Versio Iberica. II. Orationes XV, XXIV, XIX (Corpus Christianorum. Series Graeca 42, Corpus Nazianzenum 9), Turnhout - Leuven, 2000.

Metreveli H. - BezarachVili K. - Kourtsikidze T. - MeliKishVILI N. - OTKhMEZURI Th. - RAPHAVA M., Sancti Gregorii Nazianzeni Opera. Versio Iberica. III. Oratio XXXVIII (Corpus Christianorum. Series Graeca 45, Corpus Nazianzenum 12), Turnhout - Leuven, 2001.

Metreveli H. - BezarachVili K. - Kourtsikidze Th. - MeliKICHVILI N. - RAPHAVA M. - ChANIDZE M. - OTKHMEZURI TH., Sancti Gregorii Nazianzeni Opera. Versio Iberica. I. Orationes $X L V, X L I V, X L I$ (Corpus Christianorum. Series Graeca 36, Corpus Nazianzenum 5), Turnhout - Leuven, 1998.

Moreschini Cl. - Gallay P., Grégoire de Nazianze. Discours 38-41. Introduction, texte critique et notes par Cl. Moreschini, traduction par P. Gallay (Sources chrétiennes 358), Paris, 1990 (cité traduction de P. Gallay).

Mossay J. (éd.), II. Symposium Nazianzenum. Actes du colloque international, Louvain-la-Neuve, 25-28 août 1981 (Studien zur Geschichte und Kultur des Altertums. 2. Reihe : Forschungen zu Gregor von Nazianz), Paderborn - München - Wien - Zürich, 1983.

SAnspeur Cl., Sancti Gregorii Nazianzeni Opera. Versio Armeniaca. IV. Oratio VI (Corpus Christianorum. Series Graeca 61, Corpus Nazianzenum 21), Turnhout - Leuven, 2007.

SCHMIDT A. - QUASCHNING-KIRSCH M., « Die syrischen Handschriften der Homelie des Gregors von Nazianz. Repertorium mit Nachträgen und Sigelverzeichnis », Le Muséon 113, 2000, p. 87-114 (les données du tableau p. 113 sont erronées).

SchMIDT A.B., Sancti Gregorii Nazianzeni Opera. Versio Syriaca. II. Orationes XIII, XLI (Corpus Christianorum. Series Graeca 47, Corpus Nazianzenum 15), Turnhout - Leuven, 2002.

Sinko A., De traditione orationum Gregorii Nazianzeni. Pars I (Meletamata Patristica 2), Cracovie 1917.

SiRINIAN A., Sancti Gregorii Nazianzeni Opera. Versio Armeniaca. II. Orationes IV, V (Corpus Christianorum. Series Graeca 37, Corpus Nazianzenum 6), Turnhout - Leuven, 1999.

SOMERS V., Histoire des collections complètes des Discours de Grégoire de Nazianze (Publications de l'Institut Orientaliste de Louvain 48), Louvain-la-Neuve, 1997. 
ThOMSON Fr.J., «The Works of St. Gregory of Nazianzus in Slavonic », dans J. MosSAY (éd.), II. Symposium Nazianzenum, 1983, p. 119-125.

TUERlinckX L., Sancti Gregorii Nazianzeni Opera. Versio Arabica antiqua. II. Orationes I, XLV, XLIV (Corpus Christianorum. Series Graeca 43, Corpus Nazianzenum 10), Turnhout - Leuven, 2001.

VAN DonZel E., «Les 'versions' éthiopiennes des Discours de Grégoire de Nazianze », dans J. MossAY (éd.), II. Symposium Nazianzenum, 1983, p. 127-133.

VAN RoEY A. «Une attestation syriaque (VI ${ }^{\mathrm{e}}$ s.) d'un passage disputé de l'Or. 38 de Grégoire de Nazianze (BHG 1938) », Analecta Bollandiana, 114, 1996, p. 33-34.

Van Roey A. - Moors H., « Les Discours de saint Grégoire de Nazianze dans la littérature syriaque. I. Les manuscrits de la version 'ancienne' », Orientalia Lovaniensia Periodica 4, 1973, p. 122-133; «II. Les manuscrits de la version 'récente' », Ibid., 5, 1974, p. 79-125.

Wright W., Catalogue of Syriac Manuscripts in the British Museum acquired since the Year 1838, 3 vol., Londres, 18701872. 